Florida International University FIU Digital Commons

FIU Electronic Theses and Dissertations

University Graduate School

6-9-2017

\title{
Thermally-Assisted Acoustofluidic Separation for Bioanalytical Applications
}

Ata Dolatmoradi

Florida International University, adola004@fiu.edu

DOI: 10.25148 /etd.FIDC001964

Follow this and additional works at: https://digitalcommons.fiu.edu/etd

Part of the Biology and Biomimetic Materials Commons, Biomedical Devices and Instrumentation Commons, and the Biophysics Commons

\section{Recommended Citation}

Dolatmoradi, Ata, "Thermally-Assisted Acoustofluidic Separation for Bioanalytical Applications" (2017). FIU Electronic Theses and Dissertations. 3371.

https://digitalcommons.fiu.edu/etd/3371

This work is brought to you for free and open access by the University Graduate School at FIU Digital Commons. It has been accepted for inclusion in FIU Electronic Theses and Dissertations by an authorized administrator of FIU Digital Commons. For more information, please contact dcc@fiu.edu. 


\section{FLORIDA INTERNATIONAL UNIVERSITY}

Miami, Florida

\section{THERMALLY-ASSISTED ACOUSTOFLUIDIC SEPARATION FOR BIOANALYTICAL APPLICATIONS}

A dissertation submitted in partial fulfillment of the requirements for the degree of DOCTOR OF PHILOSOPHY

in

MATERIALS SCIENCE AND ENGINEERING

by

Ata Dolatmoradi 
To: Interim Dean Ranu Jung

College of Engineering and Computing

This dissertation, written by Ata Dolatmoradi, and entitled Thermally-Assisted Acoustofluidic Separation for Bioanalytical Applications, having been approved in respect to style and intellectual content, is referred to you for judgment.

We have read this dissertation and recommend that it be approved.

Arvind Agarwal

Nikolaos Tsoukias

Chunlei Wang

Bilal El-Zahab, Major Professor

Date of Defense: June 9, 2017

The dissertation of Ata Dolatmoradi is approved.

Interim Dean Ranu Jung College of Engineering and Computing

Andrés G. Gil

Vice President for Research and Economic Development and Dean of the University Graduate School

Florida International University, 2017 
(C) Copyright 2017 by Ata Dolatmoradi

All rights reserved. 


\section{DEDICATION}

To my parents,

without whom none of this would have been possible. 


\section{ACKNOWLEDGMENTS}

I would like to express my gratitude to all the people who have helped me and given me support throughout the completion of my PhD studies. First, to my parents, Robabeh and Reza, who have always loved and supported me. Of course, I could not have done this without the support and encouragement of other family members including my sisters Maryam and Marjan, my brother-inlaw Dr. Pooya Hosseini-Benhangi, my aunt Dr. Nahid Shabestary, and my uncle-in-law Dr. Abbas Kadkhodayan.

I would like to thank my advisor, Dr. Bilal El-Zahab, for guiding me and helping me reach my potential. His technical guidance, support, and encouragement were very important factors in my completing this effort. This dissertation would not have been possible without the support of my committee members Dr. Arvind Agarwal, Dr. Nikolaos Tsoukias, Dr. Chunlei Wang, and Dr. Yu Zhong, all of whom deserve special thanks for providing unwavering support, professionally and personally, along the way.

Amir and Shervin, I considered whom my best friends at FIU, deserve a special mention. I am indebted to you both for your support and friendship over these years. A very special acknowledgement goes to the love of my life Elnaz, who truly supported me during the critical last two years of my dissertation, and made me feel like anything was possible. I love you, Elnaz. Finally, I would like to acknowledge the teachers, professors, and other professionals who either directly trained me, or inspired me in some way. 
ABSTRACT OF THE DISSERTATION

THERMALLY-ASSISTED ACOUSTOFLUIDIC SEPARATION

FOR BIOANALYTICAL APPLICATIONS

by

Ata Dolatmoradi

Florida International University, 2017

Miami, Florida

Professor Bilal El-Zahab, Major Professor

Changes in the biomechanical properties of cells accompanying the development of various pathological conditions have been increasingly reported as biomarkers for various diseases and as a predictor of disease progression stages. For instance, cancer cells have been found to be less stiff compared to their healthy counterparts due to the proteomic and lipidomic dysregulations conferred by the underlying pathology. The separation and selective recovery of cells or extracellular vesicles secreted from such cells that have undergone these changes have been suggested to be of diagnostic and prognostic value.

This dissertation first describes the implementation of a stiffness-based separation of phosphatidylcholine-based vesicles using a method first introduced based on the research in this work and was dubbed thermally-assisted acoustophoresis, or thermo-acoustophoresis. By tuning the temperature, we achieved the separation of vesicles of the same size, shape, and charge but with different stiffness values. It was observed that at a specific transition point, the acoustic contrast factor of vesicles changed sign from positive to negative. This change was mainly due to change in the compressibility of the vesicles, which is inversely proportional to stiffness. The acoustic contrast temperature $\left(T_{\phi}\right)$, corresponding to the temperature at which the contrast factor switches sign, was determined to be unique to the composition of the vesicles. This unique temperature signature allowed 
us to develop this separation method of vesicles with distinct membrane stiffness with target outlet purities exceeding $95 \%$.

We have further explored the functionality of this method by experimenting with cholesterolcontaining vesicles. In cells, the cholesterol content plays a crucial role in determining stiffness. Changes in the cholesterol content in cellular membranes can be an indication of pathological disorders. We evaluated the $T_{\phi}$ of vesicles at different cholesterol molar ratios $\left(X_{\text {chol }}\right)$ and developed a multi-stage lab-on-a-chip method to accomplish for the first time the separation of a three-vesicle mixture. Using $X_{\text {chol }}=0.1,0.2$, and 0.3 vesicles, we obtained efficiencies exceeding $93 \%$. The simplicity, rapidity, and label-free nature of this approach holds promise as a diagnostic and separation tool for cells affected by diseases that affect the stiffness and extracellular vesicles such as exosomes and microvesicles. 


\section{TABLE OF CONTENTS}

CHAPTER

PAGE

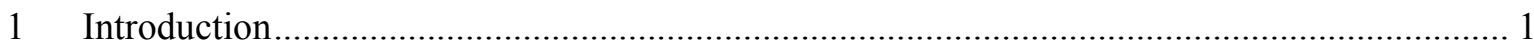

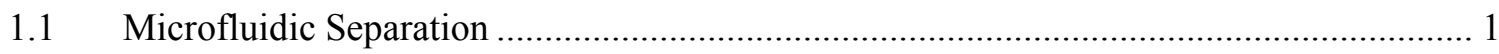

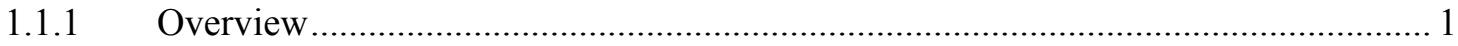

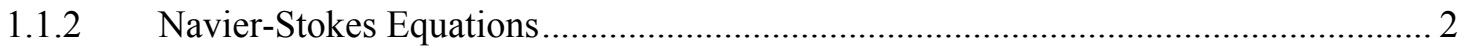

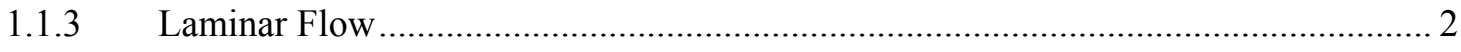

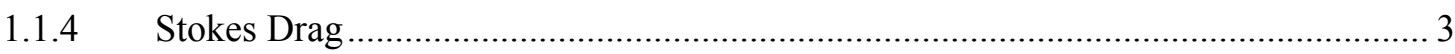

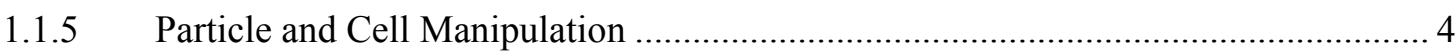

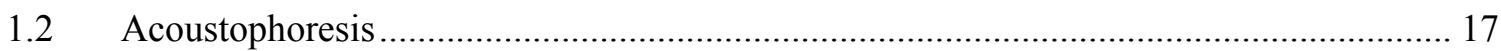

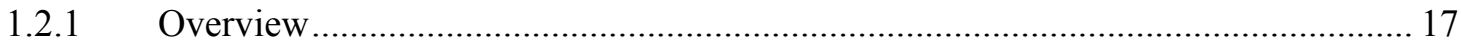

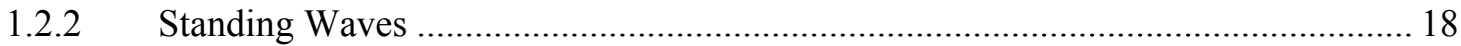

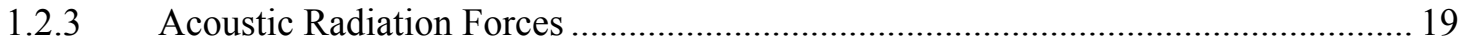

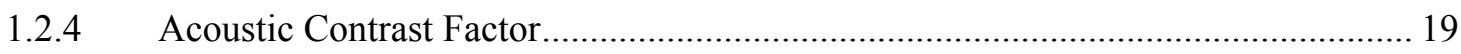

1.2.5 Acoustic Particle Manipulation............................................................................... 20

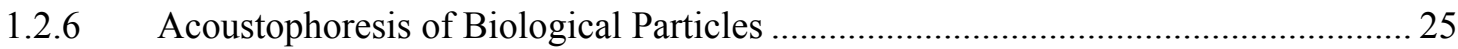

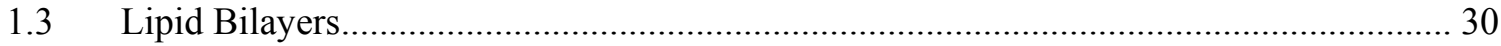

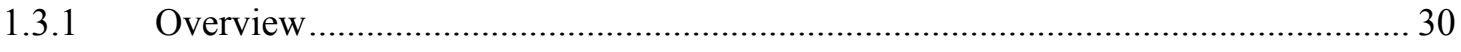

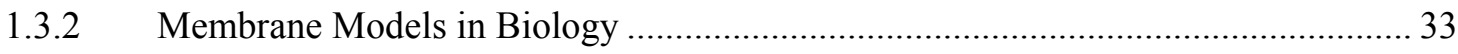

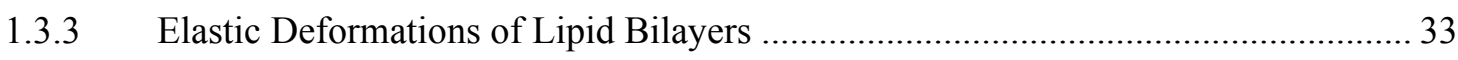

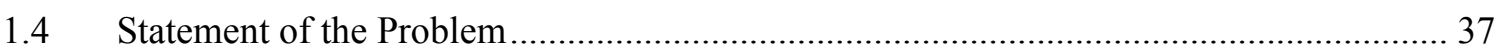

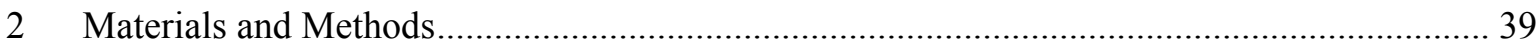

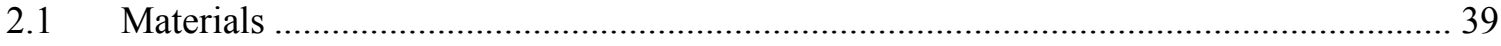

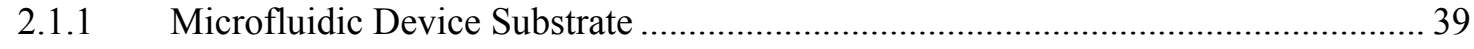

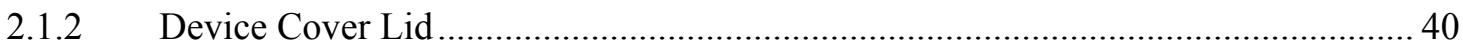

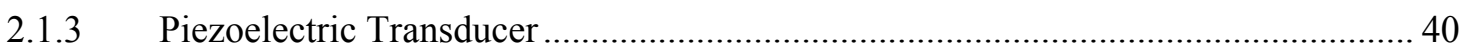

2.1.4 Lipids Used for Vesicles Preparation................................................................ 41

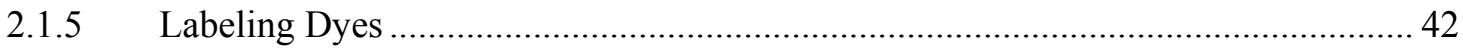

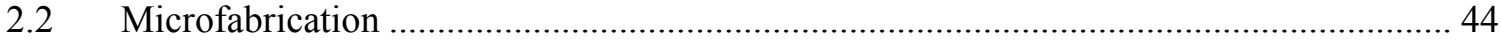

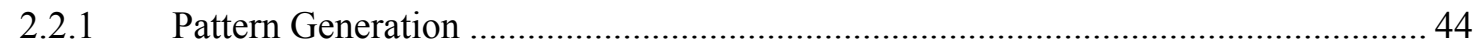

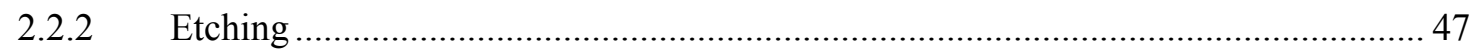

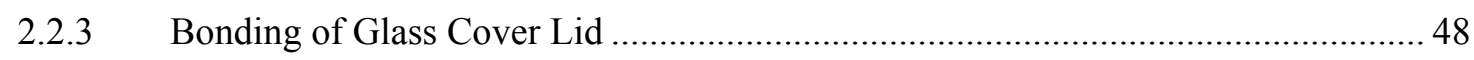

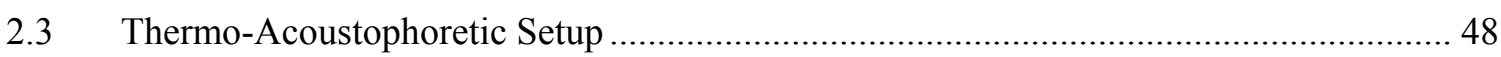

2.3.1 Temperature Management of the Device ............................................................... 50 


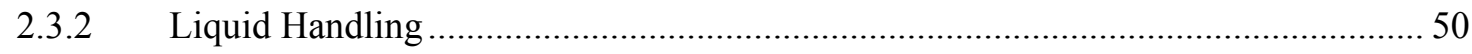

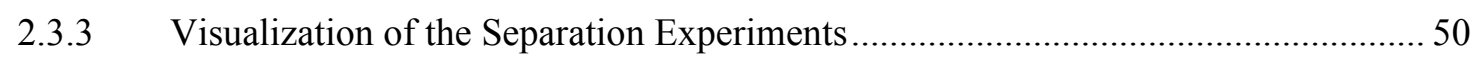

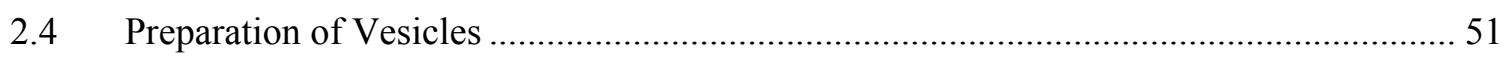

3 Thermally-Assisted Acoustophoresis of Phosphatidylcholine Vesicles ................................. 54

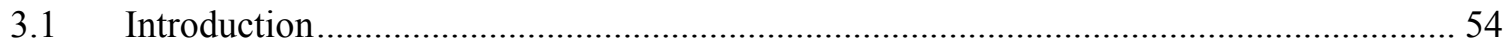

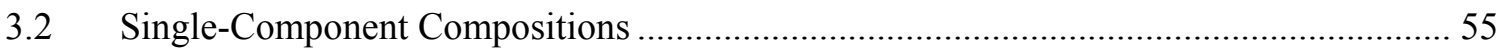

3.2.1 Thermo-Acoustophoretic Behavior of Lecithin Vesicles ........................................ 55

3.2.2 Thermo-Acoustophoretic Behavior of DMPC System ......................................... 57

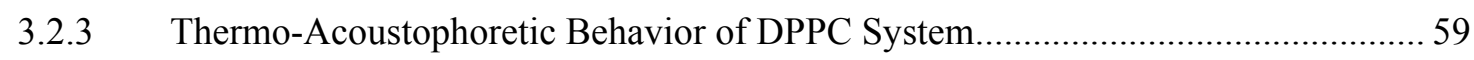

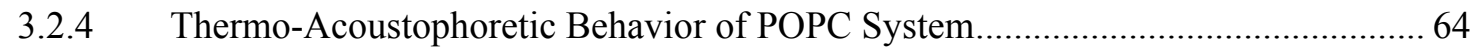

3.3 Thermo-Acoustophoretic Behavior of Vesicle with Binary Compositions ...................... 66

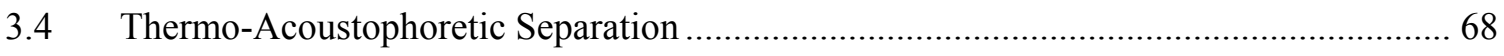

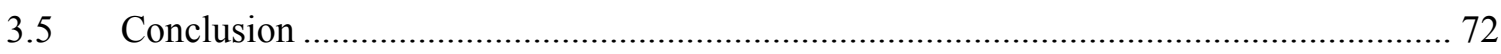

4 Thermo-Acoustofluidic Separation of Cholesterol Containing Vesicles ............................... 73

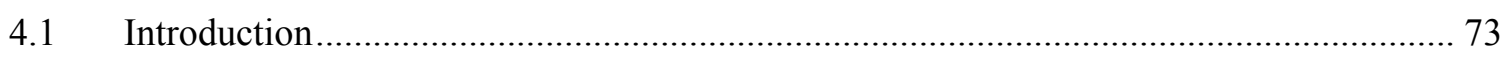

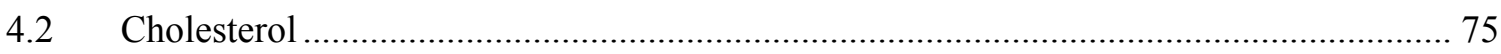

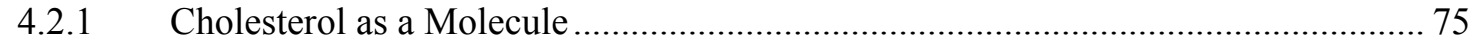

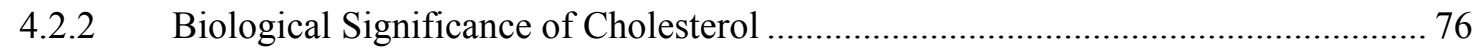

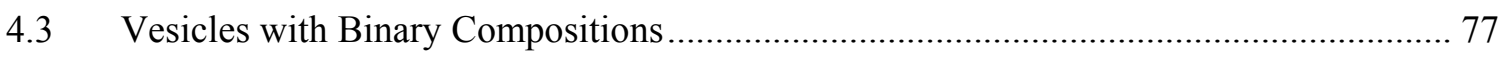

4.3.1 Thermo-Acoustophoretic Behavior of DMPC-Cholesterol System......................... 79

4.3.2 Thermo-Acoustophoretic Behavior of DPPC-Cholesterol System ........................... 80

4.3.3 Thermo-Acoustophoretic Behavior of POPC-Cholesterol System .......................... 82

4.4 Thermo-Acoustophoretic Behavior of Vesicles with Ternary Compositions ................... 83

4.5 Demonstration of Thermo-Acoustophoresis .............................................................. 84

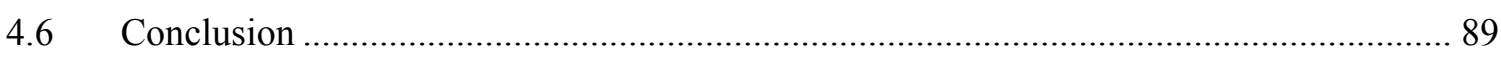

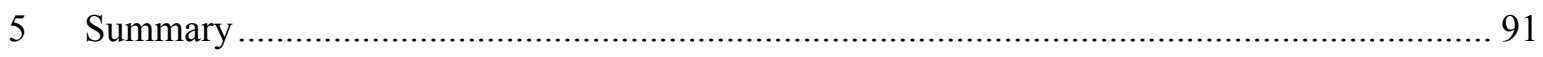

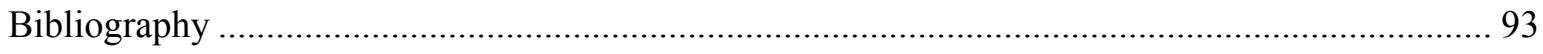

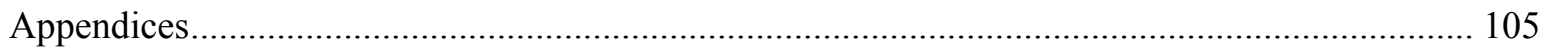

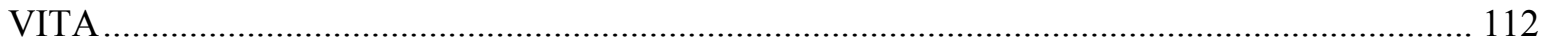




\section{LIST OF FIGURES}

FIGURE

PAGE

Figure 1.1 Schematic illustration of flow cytometry: (a) In hydrodynamic focusing, the fluid containing the cells is focused into a narrow stream in between the side sheath flows. (b) Dielectrophoretic focusing of particles in a two-dimensional configuration, using two electrodes on top and bottom of the channel (shown in yellow). In DEP only particles are affected by the electric force (Reprinted with permission from [14]).

Figure 1.2 Optical micrographs of focusing of latex particles in a flow cytometry device (a) Particles of $6 \mu \mathrm{m}$ diameter are focused in between the electrode array (dark region). (b) Timelapse images of the trajectories of the particles at different voltages. Focusing is more efficient at higher voltages (Reprinted with permission from [14])......

Figure 1.3 Microfiltration cell sorting using filtration: Schematic illustration of a microfluidic filtration device with a diffusive filter system. (Reprinted with permission from [16]) ................ 7

Figure 1.4 Schematic of insulator-based dielectrophoresis (IDEP) setup: (a) top view of the manifold, glass chip, and the microchannels. (b) Line of the applied electric field become squeezed between the insulating posts. (c) Concentration and separation of live (green) and dead (red) E. coli using IDEP. The electric field applied in the top picture is $16 \mathrm{~V} / \mathrm{mm}$, which only lead to the trapping of live cells; but on increasing the intensity to $60 \mathrm{~V} / \mathrm{mm}$ (bottom picture), differential trapping of live and dead cells is realized as two separate bands of different color. Live cells (nDEP, green) are trapped at the wider regions between the posts and dead cells (less nDEP, red) are trapped at the narrower regions. (Reprinted with permission from [20])

Figure 1.5 Simulated representation of parasitic traps in DEP devices: (a) Numerical simulation of cells inside a pDEP vial without parasitic trap cancellation. The contour profile shows the velocity of the cells due to the DEP and flow. (b) Close-up of the dashed box in (a) showing the high-electric-field regions to which cells are attracted. (Reprinted with permission from [19])

Figure 1.6 Principle of dielectrophoretic field-flow fractionation (DEPFFF): Particle velocity in the laminar parabolic flow profile can be determined according to the equilibrium height of the particle, heq, at which the DEP levitation force, FDEPz and sedimentation force, Fgrav are balanced such that $-43 \pi r 3 \rho \mathrm{p}-\rho \mathrm{mg}+F \mathrm{DEPz}=0$. (Reprinted with permission from [22])

Figure 1.7 Schematic representation of free-flow magnetophoresis. (a) Magnetic particles injected through a chamber, which is magnetically actuated perpendicular to the flow, will experience a force depending on their size and magnetic susceptibility and are therefore separated from non-magnetic particles and from each other. (Adapted from [26]) (b) Bioparticles of type A experience a positive force in the chamber focusing them into the highflux density region due to the positive magnetic susceptibility difference between them and the medium $(\chi \mathrm{pA}-\chi \mathrm{m}>0)$. Bioparticles of type B experience a negative force and are 
repelled from the high-flux density region $(\chi \mathrm{pB}-\chi \mathrm{m}<0)$. (Reprinted with permission from [23])

Figure 1.8 Optoelectronic tweezers and separation based on refractive index: (a) The fluid containing the particles is placed in between a layered structure which consists of an indium tin oxide (ITO) glass on top and a photosensitive surface made from ITO-coated glass at the bottom topped with multiple layers, namely doped hydrogenated amorphous silicon $(50 \mathrm{~nm})$, undoped hydrogenated amorphous silicon $(1 \mathrm{~mm})$, and silicon nitride $(20 \mathrm{~nm})$. The top and bottom layers are biased with an AC signal. The images formed by the illumination system and shown on the digital micromirror display (DMD) are focused onto the photosensitive layer, generating an electric field for DEP. (b) Selective separation of live cells from dead cells using the optoelectronic tweezer (OET). Randomly dispersed cells are sorted as the live cells experience a positive OET, leading them to the bright areas, and dead cells are left behind. (Adapted from [31]) (c) A guided light beam can be used to yield separation based on refractive index. Particles with greater refractive index are focused into the center due to the positive gradient force acting at the region with the highest beam intensity, while particles with smaller refractive index will experience a negative gradient force, pushing them toward the opposite direction. (Reprinted with permission from [32]).

Figure 1.9 Particle separation by deterministic lateral displacement (DLD) method: (a) Fluorescent images of the two transport modes, zigzag and displacement, for polystyrene microspheres. The gray dots are the obstacle matrix, superimposed on the fluorescence image. (b) Fluorescent microspheres with different diameters (green $(0.80 \mu \mathrm{m})$, red $(0.90 \mu \mathrm{m})$, and yellow $(1.03 \mu \mathrm{m})$ ) are separated in a matrix with varying gap size. Changing the obstacle diameters renders a matrix with varying gap sizes, as shown on the left side of the fluorescent picture. The profile on the right side shows the fluorescent intensities of the microspheres bands after separation. (Reprinted with permission from [33])

Figure 1.10 Principles of AsPFF and PFF: (a) In PFF, branch channels are identical, and so the flow distributed into them. Thus, Channels 4 and 5 are not used and the difference in the position where particles enter the channels is small. (b) In AsPFF, one channel is designed to be shorter and/or broader compared to the other ones and the flow is distributed asymmetrically, hence the name of the method. Therefore, all the channels are used and the difference in the position where particles enter the channels is large. In both figures, lighter color marks the liquid containing the particles, and the darker one marks the liquid without the particles. The size of the arrows represents the relative magnitude of the corresponding flow rates. (Reprinted with permission from [36]).

Figure 1.11 Schematic of a) a typical acoustic perturbation device, in which $\boldsymbol{y}$ marks the transversal direction of the channel and $\boldsymbol{x}$ represents the flow direction. b) An overlaid ultrasonic standing half-wave, its radiation force, and the positions of the node and antinode in the standing wave. Black arrows denote the direction of the momentum transfer and grey arrows denote the direction of the net force and thus the resulting motion.

Figure 1.12 Particle manipulation using ultrasonic standing waves: (a) Particles are randomly dispersed before actuation of the channel. (b) Actuation of the channel at a half-wave length resonance mode, causing the formation of a pressure node at the center line of the fluid layer shown in purple and therefore a net force in the direction of the arrows is formed in line with 
the blue line. (c) The established radiation force causes the movement of the particles towards the center node. (Reproduced with permission from [2])

Figure 1.13 Schematic of an acoustic sedimentation system: (a) Particles are randomly suspended in the chamber before actuation of the acoustic transducer. (b) Same particles in a) start to migrate towards the nodal plane of the chamber upon actuation of the acoustic transducer. (c) As the agglomeration grows, the particles clump tends to sediment under gravity. (Reproduced with permission from [2])

Figure 1.14 A continuous microfluidic system for the acoustic separation of particles from their suspending medium. Particles after separation are stayed focused in the middle of the channel until they are collected from the middle outlet. Clear fluid is separated via two side outlets. (Reproduced with permission from [46])

Figure 1.15 Acoustic washing of particles. Under laminar flow conditions, particles from one medium can be washed into the second medium through establishing an ultrasonic standing wave in the chamber forcing particles to migrate towards the second medium. (Reproduced with permission from [47]).

Figure 1.16 Enhanced capture of bacteria on biosensor surface via acoustic field. An overlaid quarter-wavelength standing wave with a half-wavelength thick reflector would enhance the capture of Bacillus subtilis var. niger spores on the surface of the immunosensor surface. (Reproduced with permission from [49]).

Figure 1.17 Size-selective acoustofluidic separation. Particles are fractionated into two or more groups in a quarter-wavelength channel as they experience different magnitudes of acoustic radiation force. The particles are often prefocused before entering the radiation field.

Figure 1.18 Acoustophoretic removal of proteins from RBC concentrates. The micrograph on the right shows the RBCs cleared from proteins drawn off from the center outlet and the original medium from the side outlets. (Reprinted with permission from [57])

Figure 1.19 a) Schematic illustration of the taSSAW device. b) In taSSAW-based cell separation, nodes and antinodes are designed to locate at an angle of inclination $(\theta)$ with respect to the flow. CTCs which are larger in size compared to WBCs experience a greater acoustic force, causing them to move in a greater distance perpendicular to the flow direction. The other force experienced by the cells is the drag force, which is again greater for the CTCs. c) Actual image of the taSSAW device. d) Mixture of HeLa cells and WBCs in the channel with the acoustic off. All the cells are driven along the lower side channel, exiting from the same outlet. e) Upon actuation of the device, HeLa cells which are larger are pushed toward the upper side channel, whereas the WBCs remain in the lower stream line, leading to the separation of the two cell types. The two zoomed-in images are time-lapsed micrographs of the outlets. (Reprinted with permission from [60]).

Figure 1.20 Principle of particle-binding cell acoustophoresis using elastomeric particles: a) Bright-field and $b$ ) fluorescent images of SA-adsorbed elastomeric particles bound to greenstained KG-1a cells. c) Complexes of the cells and elastomeric particles migrating to the antinodes when the acoustic transducer is on. d) Fluorescence intensity distributions of SA- 
adsorbed elastomeric particles (left), biotinylated polystyrene microbeads (middle), and complexes of particles (right) across the microchannel width (W). e) Fluorescence intensity profiles showing the separation of the unbound cells (left) from the bound cells (right). Fluorescent images of f) green KG-1a cells and the elastomeric particles embedded with Nile red focusing at the node and antinodes, respectively, and g) SA-adsorbed elastomeric particles bound to green, anti-CD34-biotin-labeled cells are separated from red-stained cells without anti-CD34-biotin by migrating to the antinodes and nodes, respectively. (Reprinted with permission from [62])

Figure 1.21 Schematic illustration of a biological membrane, showing a), the saturated and unsaturated lipid molecules, cholesterol, the actin network, glycolipids, glycosylphosphatidylinositol (GPI)-anchored proteins, lipidated proteins, and transmembrane proteins. The subdomains enriched in saturated lipids, cholesterol, (GPI)-anchored and lipidated proteins are known as lipid rafts, characterized by their higher packing order and decreased fluidity compared to the non-raft domains. b) Uneven component distribution in each leaflet and among the inner and outer leaflets of a biological membrane, leading to a membrane organization consisting of various small raft-like and non-raft domains. (Reprinted with permission from [76])

Figure 1.22 Schematic representation of four different modes of bilayer deformations: the stretching or expansion of the membrane, bending, thickness change, and shearing of the membrane. (Reprinted with permission from [63]) ....

Figure 1.23 Relationship between the $\mathrm{k}_{\mathrm{c}}$ for DLPC, DMPC, and DPPC, and the thickness of the hydrophobic region of the lipids, $d h$. (Reprinted with permission from [87]).....

Figure 1.24 Separation in this study is expected to be realized as each of the two types of vesicles experiences a different $\phi$ at $T$ transition, ves $1<T$ separation $<T$ transition, ves $2 \ldots . . .38$

Figure 2.1 Schematic of a transversal resonator, showing the optimum dimensions of the resonating substrate.

Figure 2.2 a) Molecular structure of Nile red. b) Fluorescence emission spectra of Nile Red in THF under visible light excitation $\left(450 \mathrm{~nm}, 7 \mathrm{~mW} \mathrm{~cm}^{-2}\right)$. (Reprinted with permission from [99])

Figure 2.3 Molecular structure of a) Laurdan, which is known to incorporate into the hydrophilic-hydrophobic interface of the membrane having its 12-carbon long tail, known as lauric acid, anchored in the acyl chains region. b) DiOC6(3) iodide molecule.

Figure 2.4 The layout of the 1-stage three-pronged microfluidic device, showing the inlet on the right side and the two outlets on the left. The dimensions are the mask-design dimensions in $\mathrm{mm}$. The drawing is not to scale to better show the details.

Figure 2.5 Orientation of the microfluidic channels' layouts on the (100) silicon wafer, yielding a trapezoid cross section for AA' and DD' and a rectangular cross section for BB' and $\mathrm{CC}^{\prime}$ after $\mathrm{KOH}$ etching. 
Figure 2.6 Different steps of the channel microfabrication: a) The photomask used for the pattern generation after the photoresist removal. b) A patterned wafer before etching. Green color is for the nitride layer. c) The same wafer, after silicon etch and nitride removal.

Figure 2.7 Schematic of the process flow of the fabrication of the microfluidic channels used as the separation device. The front side of the silicon wafer pre-coated with silicon nitride layer was first cleaned with acetone, IPA and HMDS and then was spin coated by a photoresist, followed by a soft-bake at the recommended temperature. The photoresist was subsequently exposed and developed in the developer solution. The developed pattern was etched with carbon tetrafluoride plasma to remove the revealed nitride layer. The underlying silicon material was subsequently removed via wet-etch processing in $\mathrm{KOH}$. To remove the remaining nitride layer on the surface, the wafer was processed with an aluminum etchant, followed by a cleaning in piranha solution. After rinsing and drying, the wafer was examined via optical microscopy to approve the feature quality. Finally, the wafer was cleaned carefully and anodically bonded with a borosilicate glass wafer on top.

Figure 2.8 a) Schematic exploded view of the thermo-acoustofluidic device used for the 1stage separation studies, featuring a thermoelectric transducer, piezoelectric transducer, and the heat sink. b) Actual image of the device, showing the chip along with the luer fittings and the fluidic tubing.

Figure 2.9 Optical micrographs of the DMPC vesicles: (a) Transmission-mode image showing a uniform population of vesicles (The inset shows a giant multilamellar vesicle in higher magnification) and (b) fluorescent image of the same sample.

Figure 2.10 2.5D view micrographs of the relative fluorescence intensity of the DPPC GVs revealing the lamellarity of the vesicles. The prepared vesicle batch contained both unilamellar and multilamellar GVs (Scale bar is $10 \mu \mathrm{m}$ ).

Figure 3.1 Thermo-acoustofluidic behavior of lecithin vesicles: a) at $5{ }^{\circ} \mathrm{C}$ when the acoustic transducer is off. b) at the same temperature with the transducer on. c) at $30.0{ }^{\circ} \mathrm{C}$, vesicles have migrated towards the antinodes. (scale bar is $100 \mu \mathrm{m})$....................................................56

Figure 3.2 Space-filling models of DMPC molecule with explicit hydrogen atoms: a) The ideal conformation. b) A representative spatial arrangement of DMPC molecule in a bilayer. In both figures, gray atoms are carbon, white are hydrogen, red are oxygen and yellow is the phosphorus. The nitrogen atom is not visible.

Figure 3.3 Thermo-acoustofluidic behavior of DMPC vesicles: a) at $1{ }^{\circ} \mathrm{C}$ when the acoustic transducer is off. b) at the same temperature with the transducer on. c) at $23.2{ }^{\circ} \mathrm{C}$, vesicles migrate towards the antinodes. (scale bar is $100 \mu \mathrm{m}$ ).

Figure 3.4 The skeletal and space-filling molecular models of DPPC showing the phosphocholine headgroup on the right side and the fatty acyl chains extended towards the left side. Both fatty acyl chains are saturated and nonpolar, comprising the hydrophobic moiety of the molecule. The phosphocholine molecule is on the other hand polar and therefore hydrophile. 
Figure 3.5 Time-lapse fluorescence micrographs of the transition from nodal focusing to antinodal focusing of DPPC vesicles as the temperature exceeded $37.6^{\circ} \mathrm{C}$ at time $\mathrm{t}=0 \mathrm{~s}$. The focusing was complete at time $\mathrm{t}=30 \mathrm{~s}$.

Figure 3.6 The relative values of $\phi$ for DMPC and DPPC vesicles vs. temperature; calculated (Appendix IV) using vesicle compressibility data from the literature. ${ }^{129,130} \phi>0$ indicates nodal focusing while $\phi<0$ indicates anti-nodal focusing. On the temperature axis where $\phi=0$, is the point dubbed by us the acoustic contrast temperature or $T \phi$. With $T \phi$, DMPC < $T$ separation $<T \phi$, DPPC, opposite focusing is achieved.

Figure 3.7 Change of $\phi$ with temperature for (a) DPPC vesicles, (b) considering a scenario where the adiabatic compressibility of the bulk medium retains room temperature value at various temperatures, and (c) considering a scenario where the adiabatic compressibility of the vesicles retains room temperature values at various temperatures.

Figure 3.8 Space-filling models of POPC molecule with explicit hydrogen atoms: a) The ideal conformation. The kink on the right chain is due to the double bond of the oleic acid. b) A representative spatial arrangement of POPC molecule in a bilayer.

Figure 3.9 Schematic illustration of a POPC bilayer membrane in gel (ordered) and fluid (disordered) states. During the main transition from the ordered state to the disordered state the lateral crystalline arrangement and chain order of the lipid molecules are lost. (The bilayer is surrounded on both sides by water molecules shown in pink and white. The gray atoms are the hydrogen atoms explicitly shown on the acyl chains).

Figure 3.10 The $\mathrm{T}_{\phi}$ of DMPC:DPPC binary mixtures superimposed on the thermotropic isomorphous phase diagram of the same system drawn using the data obtained from the mean value of the theoretical and experimental data obtained by electron spin resonance (ESR) and differential scanning calorimetry (DSC) studies (with solidus (dotted) and liquidus (dashed) curves). ${ }^{134,135}$ The violet circle markers denote the measured $T \phi$ during the heating experiment while the green diamond markers denote the measured $T \phi$ during the cooling curve. The heating $T \phi$ nearly overlapped with the solidus line over the molar ratio range. The error bars represent the maximum and minimum $T \phi$ observed.

Figure 3.11 Monitoring the performance of the separation experiment of 100:0 DMPC:DPPC and 70:30 DMPC:DPPC samples. (a) Optical fluorescence micrographs of 100:0 (red) and 70:30 (blue) DMPC:DPPC vesicles dispersed in the microchannel at $24.5^{\circ} \mathrm{C}$ with the acoustic transducer switched off. (b) The same vesicles after 3 seconds of the transducer switched on showing 100:0 DMPC:DPPC completed migration towards the pressure antinodes (red) and 70:30 DMPC:DPPC completed migration towards the pressure node (blue) (scale bars in both A and B images are $150 \mu \mathrm{m})$.

Figure 3.12 Performance of the separation of DMPC from 70:30 and 100:0 DMPC:DPPC: a) Relative fluorescence intensity scanned in the direction normal to the flow for both red and blue emissions in the separation experiment of 100:0 DMPC:DPPC and 70:30 DMPC:DPPC samples. The separation temperature was $25^{\circ} \mathrm{C}$, the approximate midpoint of the allowable window. b) Target outlet purities at 6 different temperatures over the allowable separation window $(T \phi$, DMPC $<T$ separation $<T \phi$, DPPC) for pure DMPC and DPPC vesicle systems. The error bars represent the standard deviation for $\mathrm{n}=3$...... 
Figure 3.13 Separation of 50:50 DMPC:DPPC (green) from 30:70 DMPC:DPPC (red) sample: a) Optical micrographs of dispersed pure 50:50 DMPC:DPPC and 30:70 DMPC:DPPC GVs at $30.7^{\circ} \mathrm{C}$ with the piezoelectric transducer off. b) The same vesicles at the same temperature after the transducer switched on showing 50:50 DMPC:DPPC GVs pushed towards the antinodes and 30:70 DMPC:DPPC GVs gathered at the center node (scale bars are $80 \mu \mathrm{m}$ ). c) Performance of the separation at the midpoint temperature shown as the fluorescence intensity scanned in the transversal direction of the channel. d) Target outlet of GVs, based on which the purity of collected vesicles was calculated.

Figure 4.1 a) The skeletal and b) the space-filling molecular models of cholesterol showing the molecule's large planar ring structure attached to one polar hydroxyl group on one side and to a short nonpolar hydrocarbon chain on the other side.

Figure 4.2 Determination of the acoustic contrast temperature, $T \phi$, (circles) for the DMPC:Chol binary mixtures . The background dashed lines represent the binary equilibrium phase diagram of the system, plotted using the data available in the literature. ${ }^{192} \mathrm{~S}$ denotes solid, Lo liquid ordered, and Ld liquid disordered phases. Diamond markers represent the $k c$ at specific $X$ chol at room temperature obtained from X-ray scattering methods. ${ }^{86}$

Figure 4.3 Determination of the acoustic contrast temperature, $T \phi$, (circles) for the DPPC:Chol system. The background dashed lines represent the equilibrium phase diagram of the system, plotted using the data from the literature. ${ }^{193}$ Diamond markers represent the $k c$, at room temperature obtained from molecular dynamic simulations. ${ }^{195}$

Figure 4.4 Determination of the acoustic contrast temperature, $T \phi$, (circles) for the binary POPC:Chol system. The background dashed lines represent the binary equilibrium phase diagram of the system, plotted using the data available in the literature. ${ }^{194}$ Diamond markers represent the bending stiffness modulus, $k c$, at specific $X \mathrm{chol}$ at room temperature obtained from vesicle fluctuation analysis studies. ${ }^{196}$

Figure 4.5 Triangular contour map of the measured $T \phi$ for the DMPC:DPPC:Chol system up to the $X \mathrm{chol}=0.3$. The coloured surface indicating the value of $T \phi$ was obtained using the data of 45 different points across the region of interest by thermo-acoustophoretic heating experiments. Of these 45 points, 38 of them were the nodes of the black triangles. The remining 7 were additional mid-node points that were also obtained to confirm the behavior in the 50:30:20 DMPC:DPPC:Chol region and to get higher resolution on the $T \phi$ behavior. Black lines that appear at every fifth contour line mark a temperature difference of $1.5{ }^{\circ} \mathrm{C}$. The overall trend in the diagram exhibits an increase in the $T \phi$ from the minimum in the region shaded purple to the maximum in either the binary 70:30 DMPC:Chol or 70:30 DPPC:Chol systems. The $T \phi$ values shown here are averages of at least three separate experiments.

Figure 4.6 Determination of the separation window for DMPC:Chol and DPPC:Chol systems by calculating the relative values of $\phi$ for a) DMPC and b) DPPC vesicles in pure state and in $X$ chol $=0.3$. The temperature windows are marked in green and are considered the temperature range within which separation could be achieved due to opposite migration direction of the vesicles (T $\phi, 100: 0$ PC: Chol < Tseparation $<T \phi, 70: 30$ PC: Chol). The relative values of $\phi$ were calculated using vesicle sound velocity measurements and compressibility data from the literature. ${ }^{199,200}$ 
Figure 4.7 Optical fluorescence micrographs of various stages of the multi-stage thermoacoustophoretic separation with (a) channel view with Peltier elements and actuators switched off showing randomly dispersed vesicles of $X \mathrm{chol}=0.1$ (green), $X \mathrm{chol}=0.2$ (red), and $X \mathrm{chol}$ $=0.3$ (blue) at $T=23{ }^{\circ} \mathrm{C}$, (b) actuator switched on to first harmonic frequency with Peltier elements remaining off showing all vesicles focusing to the nodal region, (c) schematic of the multi-stage separation channel showing the two stages of separation and the inlet/outlets, (d) first stage channel temperature set to $27^{\circ} \mathrm{C}$ leading to the migration of the $X \mathrm{chol}=0.1$ vesicles to the anti-nodal zones, (e) second stage temperature set to $31^{\circ} \mathrm{C}$ leading to the migration of the $X \mathrm{chol}=0.2$ vesicles to the anti-nodal zones. (Scale bars in all images are $80 \mu \mathrm{m}$ ).

Figure 4.8 Purity of collected vesicles samples from outlets 1, 2, and 3 at various temperature conditions of the two stages of the separation. Purities were quantified based on the intended vesicle for each outlet with $X \mathrm{chol}=0.1$ in outlet $1, X \mathrm{chol}=0.2$ in outlet 2, and $X \mathrm{chol}=0.3$ in outlet 3 . The cumulative purities were calculated for each temperature condition scenario using the purity of outlets 1,2 , and 3 . 


\section{ABBREVIATIONS AND ACRONYMS}

$\mathrm{AC}$

$\mathrm{ACF}$

AsPFF

BAW

CFD

Chol

CTC

DAPI

DC

DEP

DLD

DLPC

DMD

DMPC

DOPC

DPPC

DRIE

DSC
Alternating Current

Acoustic Contrast Factor

Asymmetric Pinched Flow Fractionation

Bulk Acoustic Wave

Computational Fluid Dynamics

Cholesterol

Circulating Tumor Cell

4',6-Diamidino-2-phenylindole

Direct Current

Dielectrophoresis

Deterministic Lateral Displacement

1,2-Dilauroyl-sn-glycero-3-phosphocholine

Digital Micromirror Display

1,2-Dimyristoyl-sn-glycero-3-phosphocholine

1,2-Dioleoyl-sn-glycero-3-phosphocholine

1,2-Dipalmitoyl-sn-glycero-3-phosphocholine

Deep Reactive Ion Etching

Differential Scanning Calorimetry 


\begin{tabular}{|c|c|}
\hline EMT & Epithelial-Mesenchymal Transition \\
\hline EPC & Egg L- $\alpha$-phosphatidylcholine \\
\hline ESR & Electron Spin Resonance \\
\hline FACS & Fluorescence Activated Cell Sorting \\
\hline FFA & Free Flow Acoustophoresis \\
\hline FITC & Fluorescein isothiocyanate \\
\hline FP & Fluorescence Polarization \\
\hline GMV & Giant Multilamellar Vesicle \\
\hline GUV & Giant Unilamellar Vesicle \\
\hline GV & Giant Vesicle \\
\hline HMDS & Hexamethyldisilazane \\
\hline IC & Integrated Circuit \\
\hline $\operatorname{Ig} \mathrm{A}$ & Immunoglobin A \\
\hline IPA & Isopropyl Alcohol \\
\hline ITO & Indium Tin Oxide \\
\hline $\mathrm{KOH}$ & Potassium hydroxide \\
\hline LOC & Lab on a Chip \\
\hline LUV & Large Unilamellar Vesicle \\
\hline MEMS & Micro Electro Mechanical System \\
\hline
\end{tabular}




\begin{tabular}{|c|c|}
\hline MLV & Multilamellar Large Vesicle \\
\hline NS & Navier-Stokes equations \\
\hline OET & Optoelectronic Tweezer \\
\hline $\mathrm{PC}$ & Phosphatidylcholine \\
\hline PDMS & Poly(dimethylsiloxane) \\
\hline $\mathrm{PE}$ & Phosphatidylethanolamine \\
\hline PFF & Pinched Flow Fractionation \\
\hline PG & Phosphatidylglycerol \\
\hline PI & Phosphatidylinositol \\
\hline PIV & Particle Image Velocimetry \\
\hline POC & Point of Care \\
\hline POPC & 1-Palmitoyl-2-oleoyl-sn-glycero-3-phosphocholine \\
\hline PRF & Primary Acoustic Radiation Force \\
\hline PS & Phosphatidylserine \\
\hline PtdCho & Phosphatidylcholine \\
\hline PtdEtn & Phosphatidylethanolamine \\
\hline PtdGro & Phosphatidylglycerol \\
\hline PtdIns & Phosphatidylinositol \\
\hline PtdSer & Phosphatidylserine \\
\hline
\end{tabular}




$\begin{array}{ll}\text { PZT } & \text { Lead Zirconate Titanate } \\ \text { RBC } & \text { Red Blood Cell } \\ \text { SA } & \text { Streptavidin } \\ \text { SAW } & \text { Surface Acoustic Wave } \\ \text { SSAW } & \text { Standing Surface Acoustic Wave } \\ \text { SRF } & \text { Secondary Acoustic Radiation Force } \\ \text { SUV } & \text { Small Unilamellar Vesicle } \\ \mu-\mathrm{TAS} & \text { Micro Total Analysis System } \\ \text { taSSAW } & \text { Tilted-Angle Standing Surface Acoustic Wave } \\ & \\ \text { THF } & \text { Tetrahydrofuran } \\ & \text { White Blood Cell }\end{array}$




\section{Introduction}

\subsection{Microfluidic Separation}

\subsubsection{Overview}

Microfluidics is a rapidly-growing interdisciplinary field that deals with the study of the characteristics, manipulation, and mixing of liquids, or more properly fluids, in extremely small volumes, ranging from microliter to picoliter or even smaller. ${ }^{1}$ At these scales (10 to $100 \mu \mathrm{m}$ of channel size), fluids begin to exhibit some unique and unprecedented properties mainly because the Reynolds number, which characterizes the nature of the flow regime as a ratio of the inertial forces to viscous forces, becomes extremely small. ${ }^{2}$ Small Reynolds number bring about organized laminar flow streams which are associated with high momentum diffusion and low momentum convection, making diffusion the primary mode of mixing in the channel. Furthermore, in contrast to fluid flow in the macroscale where factors like turbulence, inertia and friction are significant, in microfluidics due to the large surface to volume ratios factors like surface tension and fluidic resistance become dominant. ${ }^{3}$ The study of the behavior of fluids under these conditions and devising new methods for making use of these properties for new applications is the main subject of microfluidics. Microfluidics not only provides an opportunity to study microscale phenomena, but also enables the development of many new technologies ranging from hand-held fielddeployable biomedical devices to assays capable of performing analytical studies at a very small scale. ${ }^{4}$ As measured by the number of published papers, overall productivity of scientific research and development in microfluidics have increased dramatically in the past 20 years. This chapter covers the fundamentals and applied aspects of microfluidics and highlights some of the latest developments in microfluidic technologies, especially for biological sample processing, by covering two decades of research in this field. 


\subsubsection{Navier-Stokes Equations}

The flow of incompressible liquids in continuum state, which is often the case in many microfluidic setups,${ }^{2}$ can be described by the Navier-Stokes equations (NS). The NS equations, named after Claude-Louis Navier and George Gabriel Stokes, describe the flow of viscous fluids. These equations are derived by applying the Newton's second law to the motion of a fluid, assuming that the stress in the fluid is the sum of a pressure term and a diffusing viscous term, with the latter being proportional to the gradient of velocity. The flow equations of an incompressible fluid are defined as: ${ }^{5}$

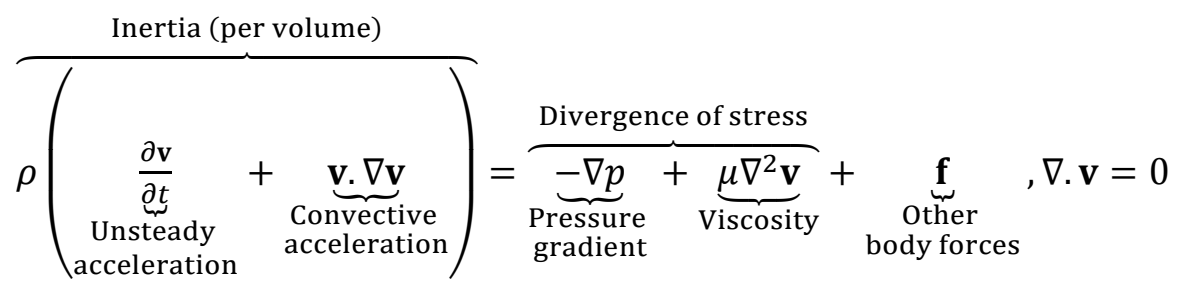

where $\rho$ is the density of the fluid, $\mathbf{v}$ is the flow velocity vector, $\nabla$ is the del operator, $p$ is the pressure in the fluid $\mu$ is the dynamic viscosity of the fluid, $\mathbf{f}$ represents forces acting on the body, for example gravity, inertial, electrostatic, acoustic, etc. The terms on the left-hand side of the equation correspond to the inertial forces, while those on the right-hand side describe the pressure forces, viscous forces, and the external forces applied to the fluid, respectively. If solved for a given set of boundary conditions (e.g. inlets, outlets, and the walls), the velocity and pressure of the fluid can be calculated in the given geometry. Since the convective term is nonlinear, Eq. 1 is often solved numerically through different methods, ${ }^{6}$ such as finite element method (FEM), finite volume method (FVM), or by using computational fluid dynamics (CFD) software packages.

\subsubsection{Laminar Flow}

When a fluid, in a given setup, flows in layers parallel to one another with minimal lateral mixing or cross-current between the layers, the flow is called laminar or streamline flow. ${ }^{7}$ In fluid mechanics, the nature of a fluid flow is often characterized by a characteristic dimensionless 
number known as Reynolds number $(R e)$, which expresses the ratio of inertial forces to viscous forces and it is defined as ${ }^{8}$

$R e=\frac{L \rho V_{\text {average }}}{\mu}$

where $L$ is the most relevant length scale, $\rho$ is the density of the fluid, $V_{\text {average }}$ is the average velocity of the flow, and $\mu$, as was mentioned before, is the viscosity of the fluid. Reynolds numbers greater than 2000 are typically associated with the transition of the flow from laminar to a turbulent regime. As the dimensions of channels are very small in microfluidics, the $R e$ of the flow is very small (Re $\ll 100)$, rendering a completely laminar flow. ${ }^{9}$ Since the Reynolds numbers are extremely small in microfluidic channels, the ratio of the inertial forces compared to the viscous forces is very small and therefore they can be considered as negligible when solving the NS equations. ${ }^{2}$ In other words, the full NS equations can be linearized as Stokes equations, which is much more computation-effective. Therefore, if the flow is assumed to be steady, we would have: ${ }^{10}$

$\nabla p=\mu \nabla^{2} \mathbf{v}+\mathbf{f}, \nabla \cdot \mathbf{v}=0$

where $p$, as mentioned earlier, is the fluid pressure, $\mathbf{v}$ is the fluid velocity, and $\mathbf{f}$ is the additional forces acting on the flow.

\subsubsection{Stokes Drag}

The frictional force or the drag force exerted on spherical particles with very small Reynolds numbers can be calculated by the Stokes' law, which describes a flow in which the advective inertial forces are small compared to viscous forces. The Stokes drag force a particle in such a flow is described by ${ }^{10}$

$F_{y}^{\mathrm{drag}}=-6 \pi r \mu v_{y}$

where $\mu$ is the viscosity of the medium and $v_{y}$ is the velocity of the particle along the direction $y$. In SI units, drag force is usually given is Newtons, viscosity in Pascal seconds, $r$ in 
meters and velocity in meters per second. It will be shown later that the migration velocity of a particle in a microfluidic channel along the direction $y$ can be found by the balancing out the external force along the direction $y$ with the Stokes' drag force. ${ }^{11}$

\subsubsection{Particle and Cell Manipulation}

In clinical diagnostics, life sciences, biomedical research, and many other analytical studies, detection and isolation of a particular cell population from a heterogenous mixture of cells is of great interest. ${ }^{12}$ This task has however proven to be challenging as the method of choice must be gentle, rapid and capable of differentiating cells with various physiochemical properties. ${ }^{2,12}$ In this context cell soring refers to the fractionation of a heterogeneous population of cells into purified subpopulations of homogeneous cells, while cell separation is only the isolation of particular cell(s) from a heterogenous mixture.

Although conventional sorting methods such as flow cytometry, differential centrifugation, and filtration are rapid and efficient in sorting cells on the macroscale, they suffer from several drawbacks, including the low resolution, high cost, and sample dilution and dispersion. Various attempts have been made to resolve these issues which have resulted in a number of miniaturized systems working based on microfluidic platforms. Microfluidic systems have been found to outperform their conventional macroscale counterparts given their capability of processing samples of much less volume size and the inherent precise control over the cellular milieus offered by them. Furthermore, these systems have very high throughputs as they can be easily scaled-up in multiple parallel modules and integrated with post analytical processing.

This chapter confines itself to underlining some of the most common microfluidic methods which are either a miniaturized adaptation of a conventional macroscale method or a novel setting harnessing the microscale properties to yield separations, with emphasis on the more mainstream commercial developments in the latter types. 


\subsubsection{Flow Cytometry}

Flow cytometry is one of the most commonly used cell sorting methods, which is capable of counting, analyzing, and sorting microparticles including cells suspended in a fluid and flowing through an optical or electrical detection sensor. ${ }^{2}$ Although rapid and unique in offering the possibility of a reliable single-cell analysis, flow cytometry systems have a number of drawbacks: system complexity, large size, high cost, multiple pretreatment steps, relatively high volume of starting samples, and, perhaps most significantly, the need of highly professional staff. ${ }^{13}$ These features have encouraged investigations into novel microfluidic systems, aiming at lowering the cost of the analysis, simplifying the operation and decreasing the size of the device. Flow Cytometry systems work based on the detection of single cells prelabeled with antibodies, magnetic labels, or fluorescent dyes focused through a detection region. Focusing of the cells is realized through either passive methods utilizing channels confining the cells into a single line or active methods using hydrodynamic, electrical, magnetic, or ultrasonic forces. Of the active focusing methods, hydrodynamic forcing is the most common one (Figure 1.1a). In a typical configuration, the fluid containing the cells is pumped through a central channel of a cross-like junction of channels. As the flow in the main channel and the sheath flow channels meet at the junction, the central channel's flow is focused into a narrow layer. ${ }^{14}$ The benefit to be gained from such a setting is that the velocities of the particles are independent of the flow and particle properties as well as the channel material. ${ }^{2}$ The main drawback of hydrodynamic focusing is the need for a precise control over the flows for an efficient focusing and ensuring that the particles are not driven into the sheath fluid. Electrokinetic focusing is an alternative method which utilizes a DC electric field of high voltages $(\sim 1 \mathrm{kV})$ to render the particles focused into a single line, exploiting a cross-shaped architecture, analogous to hydrodynamic focusing. As the main channel meet with the side channels, the flow in it is focused into a narrow stream in between the sheath flows. The other method used for focusing of the particles in flow cytometry is dielectrophoresis (DEP), which is 
based on the movement of polarized particles in a nonuniform electric field ${ }^{14}$ (Figure 1.2). In contrast to hydrodynamic focusing, in DEP the acting force is exerted on the particles rather than of the fluid, obviating the need for side flows to focus the particles into a single line.

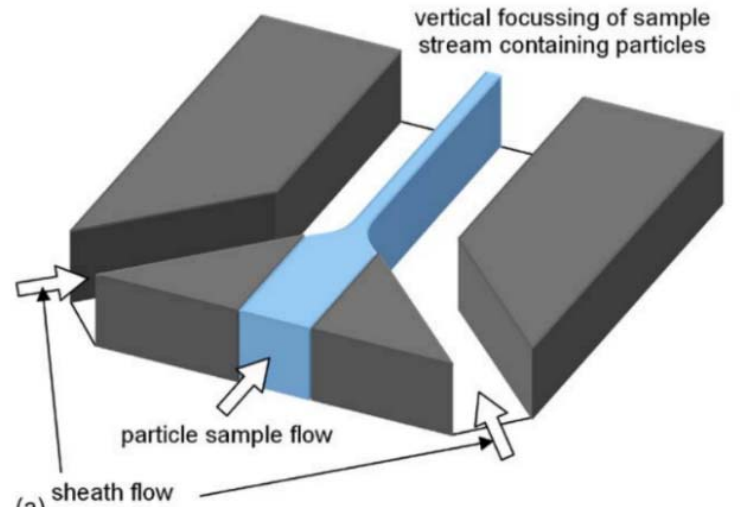

(a) sheath flow

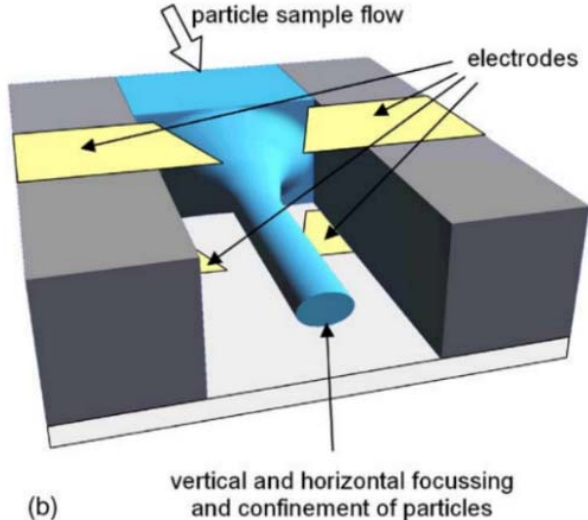

(b) and confinement of particles

Figure 1.1 Schematic illustration of flow cytometry: (a) In hydrodynamic focusing, the fluid containing the cells is focused into a narrow stream in between the side sheath flows. (b) Dielectrophoretic focusing of particles in a two-dimensional configuration, using two electrodes on top and bottom of the channel (shown in yellow). In DEP only particles are affected by the electric force (Reprinted with permission from [14]).

Cells lined up though either of the mentioned methods are then deflected from the main stream for subsequent analysis. The deflection and sorting is realized though control of either the fluid by electrokinetic and/or hydrodynamic forces or the particles. Optical forces can also be used for cell sorting in flow cytometry.,
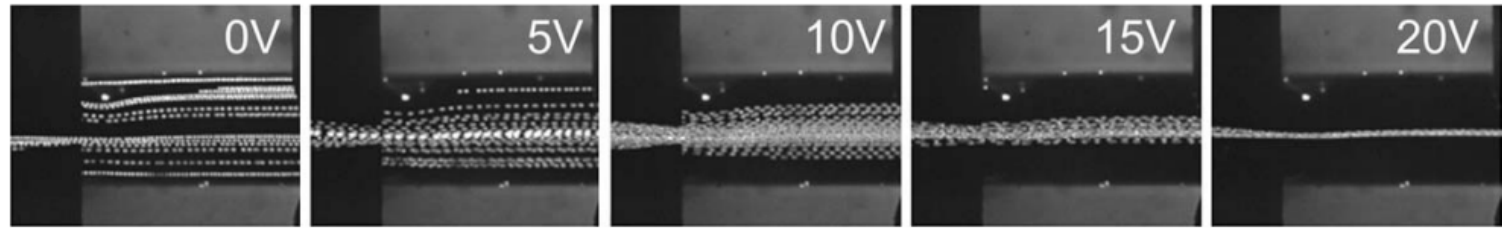

Figure 1.2 Optical micrographs of focusing of latex particles in a flow cytometry device (a) Particles of $6 \mu \mathrm{m}$ diameter are focused in between the electrode array (dark region). (b) Time-lapse images of the trajectories of the particles at different voltages. Focusing is more efficient at higher voltages (Reprinted with permission from [14]). 


\subsubsection{Microfluidic Filtration}

Filtration is a sorting technique which works based on the size-selective separation of particles. Miniaturized filtration systems with arrays of features of different geometries, made using microfabrication techniques, have been successfully implemented in separating heterogenous populations of cells. ${ }^{2}$ The main advantage offered by microfluidic filtration systems is that they do not require specific buffers. These systems however suffer from several drawbacks, including the pressure build-up and clogging of the filters with larger volume samples, irreversibility of the operation, and complexity of the fabrication process. ${ }^{15}$ A number of initiatives have been undertaken to resolve these issues in microfiltration systems, including using a filter parallel to the fluid flow direction (Figure 1.3). ${ }^{16}$ In such a system a central flow brings the cells into the device with larger cells stay suspended in the main flow and smaller ones driven into the lateral stream by high-tangential filters. Although such configuration is much less prone to clogging, it suffers from low efficiency rates especially with samples of heterogenous size and mechanical properties.

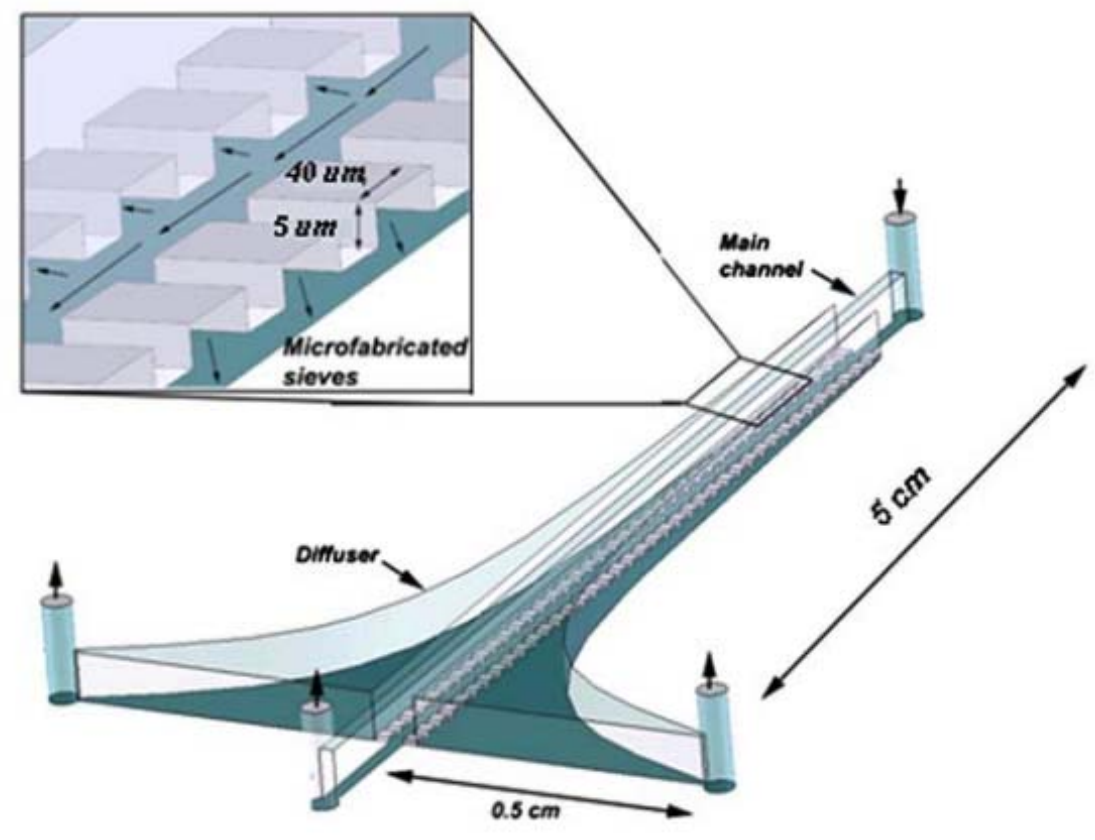

Figure 1.3 Microfiltration cell sorting using filtration: Schematic illustration of a microfluidic filtration device with a diffusive filter system. (Reprinted with permission from [16]) 


\subsubsection{Electrical Forces}

Electrophoresis and dielectrophoresis are the electrical forces used in microfabricated cell sorting systems. ${ }^{2}$ Electrophoretic sorting is based on the interaction of an electric field with a charged cell, while DEP, as was mentioned earlier, is based on the polarization of cells in a fluid sample. ${ }^{17}$ Both effects can be employed on miniaturized systems to separate mixtures of cells into their constituent cell types. Two main advantages that using electric forces offer is first the simple fabrication of the electrode using standard microfabrication techniques and the straightforward control of electric forces using inexpensive power supplies. Electrophoresis has been successfully demonstrated as a separation method for bacteria and mammalian cells in some applications. It however suffers from the limitation of the systems that can be separated by this method as separation is merely based on the charge to size ratio. A case in point is the separation of different cell types or viable and nonviable cells.

DEP, first described by Pohl, ${ }^{18}$ runs based on the electrokinetic motion of polarized particles in a non-uniform field. The force exerted on the particles is dependent on the size of the particle and the difference in the conductivity of the particle and its medium. Therefore, in contrast to electrophoresis which merely works based on the charge to size ratio of the particles, DEP is dependent on both the properties of the particles and their medium, rendering a more selective system. The force $(\mathbf{F})$ acting on a particle in DEP is given by: ${ }^{19}$

$\mathbf{F}=4 \pi \varepsilon_{\mathrm{m}} \operatorname{Re}(C M) r^{3}(\mathbf{E} \cdot \nabla) \mathbf{E}$

where $\varepsilon_{\mathrm{m}}$ is the permittivity of the medium, $r$ is the particle radius, and $\mathbf{E}$ is the electric field. $\operatorname{Re}(C M)$ in Eq. 5 is the real part of the Clausius-Mossotti factor, which expresses the relative polarizability of the particle and its medium: ${ }^{19}$

$C M=\operatorname{Re}\left(\frac{\varepsilon_{\mathrm{p}}^{*}-\varepsilon_{\mathrm{m}}^{*}}{\varepsilon_{\mathrm{p}}^{*}+2 \varepsilon_{\mathrm{m}}^{*}}\right)$ 
where subscript (p) refers to the particle and (m) refers to the medium. $\varepsilon^{*}$ is the complex permittivity, which is a function of the frequency of the field and also of the permittivity and conductivity of the material and is given by: ${ }^{19}$

$\varepsilon^{*}=\varepsilon+i \sigma / \omega$

where $\sigma$ is the conductivity of the material and $\omega$ is the frequency of the electric field. The migration direction of the particles in DEP, towards the regions with high electric field strength (positive DEP or $\mathrm{pDEP}$ ) or low electric field strength (negative $\mathrm{DEP}$ or $\mathrm{nDEP}$ ), depends on the sign of the $C M$, where a positive $C M$ affords $\mathrm{pDEP}$ and a negative one affords $\mathrm{nDEP}$.

Cells, in particular, can be manipulated using DEP given they often exhibit dielectric properties when placed in an external field. In the most common DEP system, the forces arise by the nonuniformities of an electric field generated by an array of electrodes in a microfluidic system. Insulator-based dielectrophoresis (IDEP), or electrodeless DEP or multigradient DEP in some contexts, is an alternative approach which has recently come to attention of the microfluidic community.

In IDEP, electrodes are placed at the inlet and outlet of the channel creating an electric field, which is partially disrupted in by the insulating materials in the channel. If the created field has a direct-current component, an electrokinetic flow is generated, obviating the need for external forces. DEP in its most common configuration, batch processing, applies forces which trap the cells of interest for further processing, while the other cells can pass through the channel. Batch processing is however problematic in the sense that the flow control and cell handling after separation is difficult. Therefore, efforts have been put into developing a continuous cell sorting system. DEP in its original setting uses the mobilities of cells to yield separation. However, alternative methods such as labeling can also be used for the separation of biological systems. For instance, E. coli bacteria with a specific marker has been shown to be separable from a mixture of bacteria of the same type using beads with an antibody developed for the marker. ${ }^{20}$ 


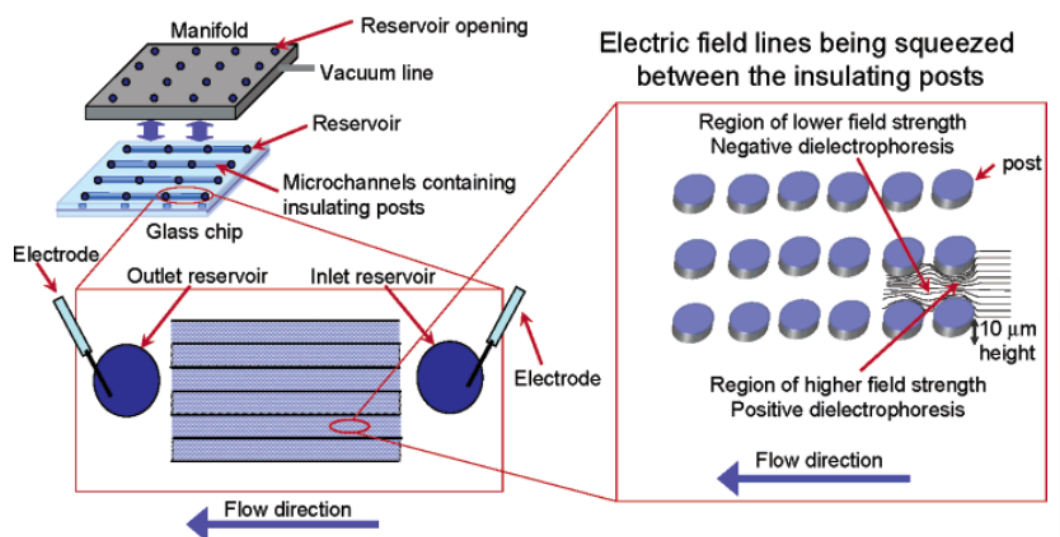

(a)

(b)

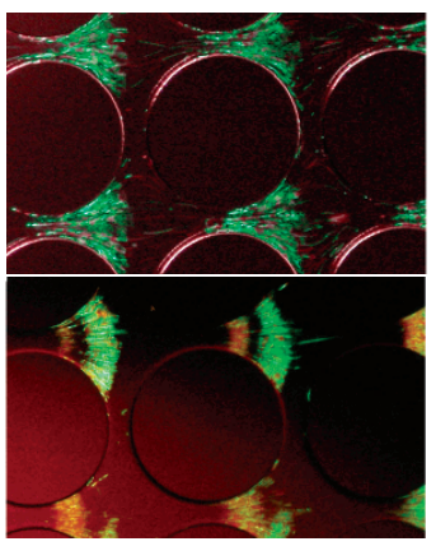

(c)

Figure 1.4 Schematic of insulator-based dielectrophoresis (IDEP) setup: (a) top view of the manifold, glass chip, and the microchannels. (b) Line of the applied electric field become squeezed between the insulating posts. (c) Concentration and separation of live (green) and dead (red) E. coli using IDEP. The electric field applied in the top picture is $16 \mathrm{~V} / \mathrm{mm}$, which only lead to the trapping of live cells; but on increasing the intensity to $60 \mathrm{~V} / \mathrm{mm}$ (bottom picture), differential trapping of live and dead cells is realized as two separate bands of different color. Live cells (nDEP, green) are trapped at the wider regions between the posts and dead cells (less nDEP, red) are trapped at the narrower regions. (Reprinted with permission from [20])

In most DEP devices, a single-frequency $\mathrm{AC}$ voltage is used between the electrodes. In an alternative configuration, however, it is possible to apply voltages of different frequencies, given that the DEP forces are superimposable. A DEP with multiple frequencies in the same device is known as multiple frequency dielectrophoresis (MFDEP), which is mainly used to minimize the parasitic traps formed due to the distortions present in the electric field. ${ }^{19}$ Figure 1.5 shows how parasitic traps can adversely affect the function of the DEP device. ${ }^{19}$
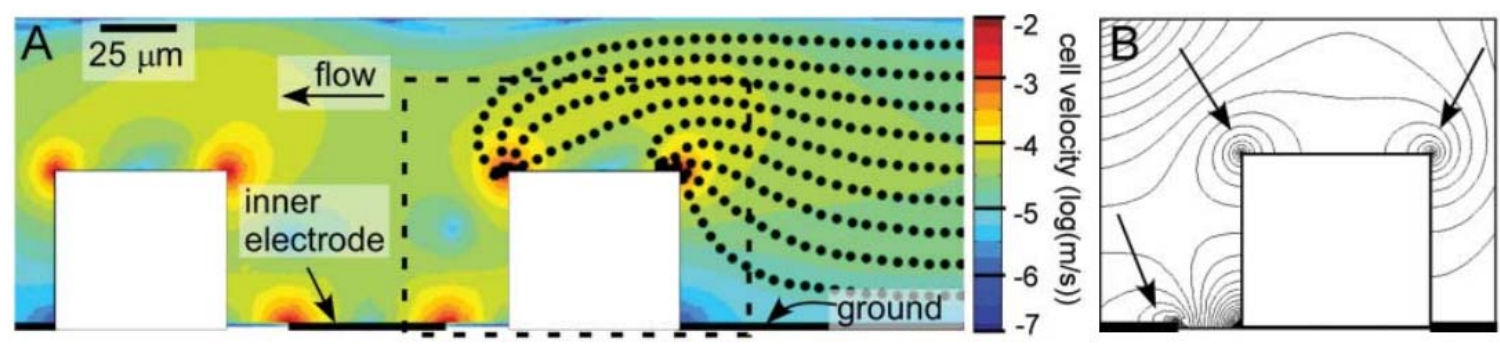

Figure 1.5 Simulated representation of parasitic traps in DEP devices: (a) Numerical simulation of cells inside a pDEP vial without parasitic trap cancellation. The contour profile shows the velocity of the cells due to the DEP and flow. (b) Close-up of the dashed box in (a) showing the high-electric-field regions to which cells are attracted. (Reprinted with permission from [19]) 
Dielectrophoretic field flow fractionation (DEPFFF) is another continuous separation in which the cells brought into the chamber are separated by the DEP force applied perpendicular to the flow direction. ${ }^{21}$ Harnessing the dielectric properties of the cells, negative DEP positions the cells into different regions of a parabolic flow (Figure 1.6). It can also switch the cells from one laminar flow to another or to side flows eventually driven into the outlets. Some of the advantages offered by DEP in cell separation applications are the large numbers of cells that can be processed via this method and the relatively large forces created by using stationary electrodes working at low voltages. The main benefit gained from separations using DEP is however the label-free nature of the separation. Main disadvantage of DEP is that the sample must be diluted to provide a lowconductivity buffer. ${ }^{21}$

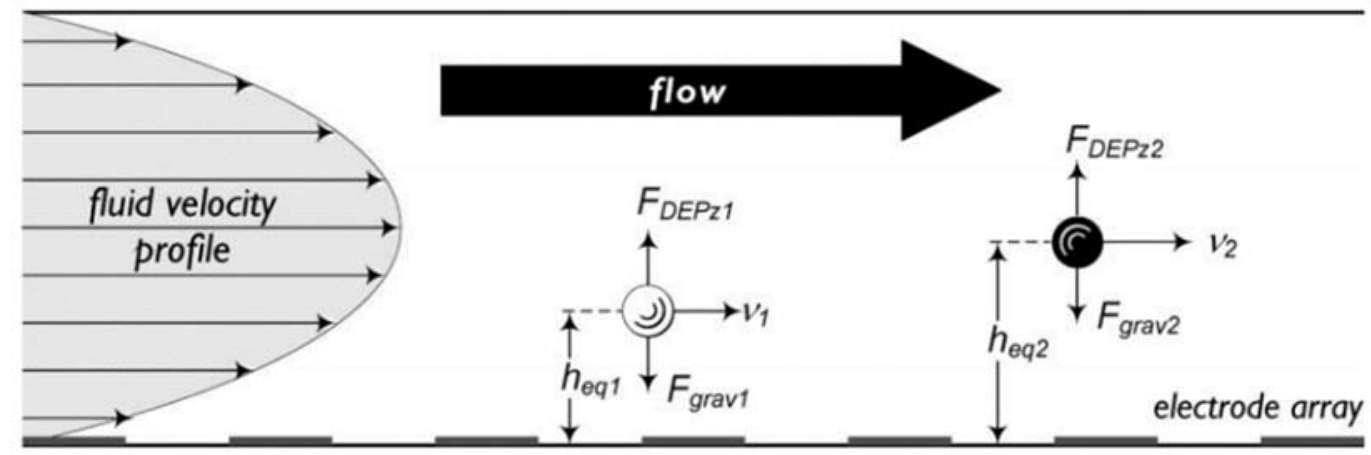

Figure 1.6 Principle of dielectrophoretic field-flow fractionation (DEPFFF): Particle velocity in the laminar parabolic flow profile can be determined according to the equilibrium height of the particle, $\boldsymbol{h}_{\text {eq }}$, at which the DEP levitation force, $F_{\mathrm{DEPz}}$ and sedimentation force, $F_{\text {grav }}$ are balanced such that $-\frac{4}{3} \pi r^{3}\left(\rho_{\mathrm{p}}-\rho_{\mathrm{m}}\right) g+F_{\mathrm{DEPz}}=0$. (Reprinted with permission from [22])

\subsubsection{Magnetic Forces}

Magnetic fields can be used to manipulate cells based on either their intrinsic magnetic properties (e.g. iron in erythrocytes) or by binding them to a magnetic material, which is known as magnetically activated cell sorting. ${ }^{2,23}$ The latter has especially attracted great interest as polymeric magnetic beads with an iron material core are now readily available. Magnetophoresis or 
magnetically induced movement of particles and their separation, realized based on different magnetic susceptibility of particles, has been also used as a method for the separation of cells by retaining the magnetized cells inside the separation chamber, while allowing the other cells pass through the chamber. ${ }^{24}$

Analogous to DEP, magnetophoresis can also be operated in batch and continuous modes, although it is much more selective due to the fact that many materials are not affected by magnetic fields. An alternative approach for trapping cells using magnetic fields is creating immunocapture beds which can be formed and released preventing the channel from clogging. ${ }^{25}$ In continuous mode, a nonuniform magnetic field perpendicular to the flow direction is applied such that a gradient of the field is created in the chamber. As the containing the particles is pumped through the inlet of the chamber particles which are not affected by the field will continue their trajectory toward the outlet, while the magnetized ones will bind to the magnetic field and remine in the channel. Any difference in magnetic susceptibility or size of the particles make them deflect at a different angle from the flow, yielding to their separation based on size or composition. ${ }^{2,23}$
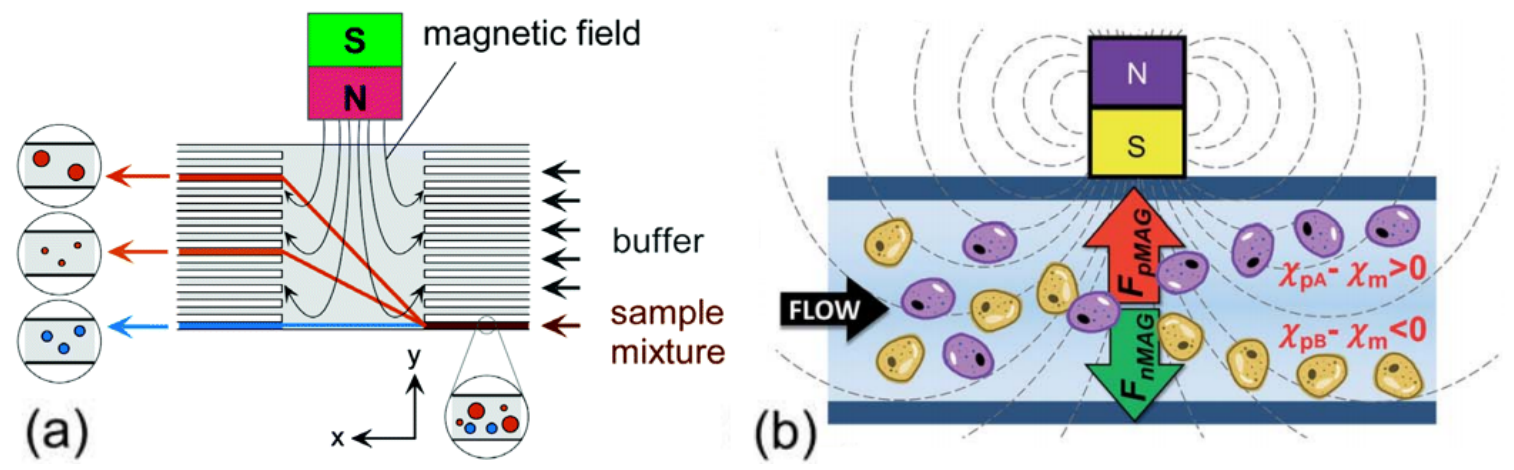

Figure 1.7 Schematic representation of free-flow magnetophoresis. (a) Magnetic particles injected through a chamber, which is magnetically actuated perpendicular to the flow, will experience a force depending on their size and magnetic susceptibility and are therefore separated from non-magnetic particles and from each other. (Adapted from [26]) (b) Bioparticles of type A experience a positive force in the chamber focusing them into the high-flux density region due to the positive magnetic susceptibility difference between them and the medium $\left(\chi_{\mathrm{pA}}-\chi_{\mathrm{m}}>0\right)$. Bioparticles of type $\mathbf{B}$ experience a negative force and are repelled from the high-flux density region $\left(\chi_{\mathrm{pB}}-\chi_{\mathrm{m}}<0\right)$. (Reprinted with permission from [23]) 


\subsubsection{Acoustic Forces}

Acoustic waves or more commonly ultrasonic standing waves can be used to separate particles and cells based on their inherent physical properties such as density and compressibility. Manipulation using ultrasonic waves is simple and do not require any labeling or pretreatment. ${ }^{27}$ In an ultrasonic standing wave, a pressure node is created which attracts particles and cells. Analogous to DEP, particle can experience a positive or negative force depending on their properties, which can be used to trap particles, concentrate them, or separate them from particles of different properties. ${ }^{11,28}$ Concepts of acoustically actuated separation and acoustophoresis are discussed more fully in the next chapter which traces the emerging role of the acoustophoresis in cell separation applications, and as a potential method for stiffness based separation of bioparticles.

\subsubsection{Optical Forces}

Optical tweezers, developed in the $1970 \mathrm{~s},{ }^{29}$ are particle manipulation instruments which work based on the pressure from a focused laser beam to trap and manipulate particles including cells. ${ }^{30,31}$ The main advantage offered by optical tweezers is their extremely high precision in manipulating particles. Other than that, the capability of holding several particles by a single beam, noninvasiveness, and the label-free nature are the other advantages of this method.

The criteria based on which a particle or cell responds to the radiation field are the size, shape, refractive index, and composition of the particle. The throughput of this method is however low as the number of the cells that can be processed at a given time is limited and the scale-up is difficult. Furthermore, the position of the particles should be specified beforehand and since the tweezers are essentially custom-made optical microscopes with a more sophisticated optics, optical tweezers are expensive instruments. Optical tweezers suffer from several other drawbacks, including the highly trained operators and more significantly the bioeffects of the laser on the cellular viability in long-term exposure times. These drawbacks form the basis of attempts to provide augmented or alternative manipulation using optical radiation, one of which is using a 
single laser source to create multiple traps by using a beam splitter. This technique is known as holographic optical tweezers (HOT), as the beam splitter is a computer-generated hologram. HOT can control hundreds of traps in a microscale 3D space. HOT enables the creation and elimination of individual traps independently, although a static array may be more useful in some applications. An alternative approach is using a scanning laser beam with different levels of intensity which has been used for the separation of polystyrene particles. For cells, another technique known as optoelectronic tweezers have been successfully demonstrated to sort cells using optical images to generate DEP electrodes.
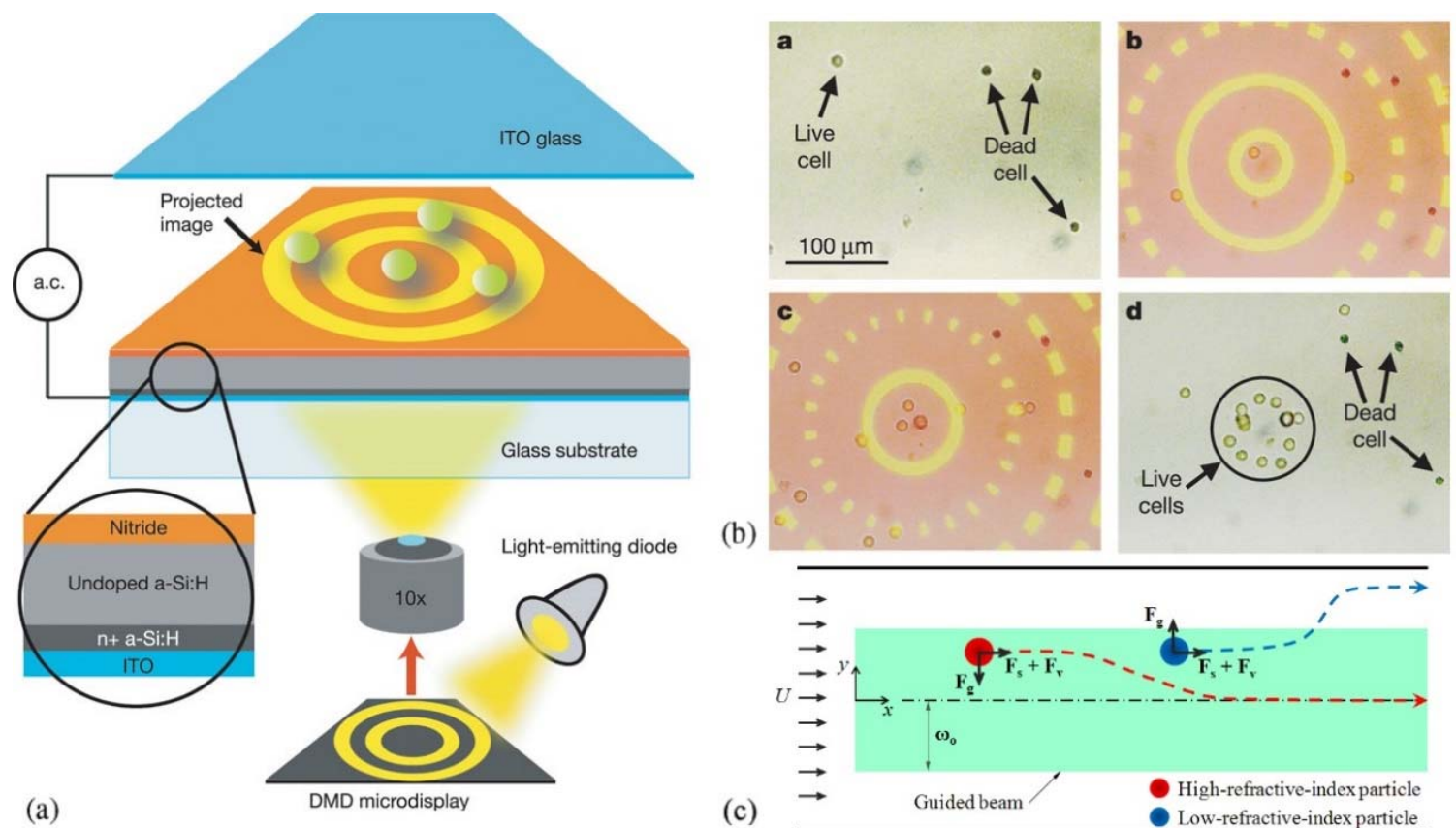

(b)

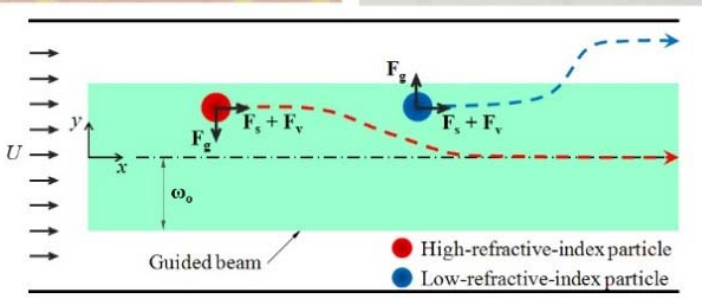

Figure 1.8 Optoelectronic tweezers and separation based on refractive index: (a) The fluid containing the particles is placed in between a layered structure which consists of an indium tin oxide (ITO) glass on top and a photosensitive surface made from ITO-coated glass at the bottom topped with multiple layers, namely doped hydrogenated amorphous silicon $(50 \mathrm{~nm})$, undoped hydrogenated amorphous silicon $(1 \mathrm{~mm})$, and silicon nitride $(20 \mathrm{~nm})$. The top and bottom layers are biased with an AC signal. The images formed by the illumination system and shown on the digital micromirror display (DMD) are focused onto the photosensitive layer, generating an electric field for DEP. (b) Selective separation of live cells from dead cells using the optoelectronic tweezer (OET). Randomly dispersed cells are sorted as the live cells experience a positive OET, leading them to the bright areas, and dead cells are left behind. (Adapted from [31]) (c) A guided light beam can be used to yield separation based on refractive index. Particles with greater refractive index are focused into the center due to the positive gradient force acting at the region with the highest beam intensity, while particles with smaller refractive index will experience a negative gradient force, pushing them toward the opposite direction. (Reprinted with permission from [32]) 
Optoelectronic tweezers are made by assembling multiple layers starting with a ground layer of indium tin oxide-coated glass, a liquid chamber, and finally a photoconductive layer on top (made from a glass coated with aluminum, amorphous silicon and silicon nitride) (Figure 1.8). ${ }^{31} \mathrm{~A}$ bias between the top and bottom electrodes is manifested as a weak electric field in the liquid chamber as most of the voltage is dropped by the high impedance of the photoconductive layer. As the light source is focused on the photoconductive layer, the local photoconductivity in that region increases, causing the formation of a nonuniform field in the liquid chamber. Given the nonuniformity of the created field, a DEP force is generated that can be used to manipulate particle inside the liquid. Since light is only used to switch the voltage drop between the liquid layer and the photoconductive layer and not for trapping the cells, optoelectronic tweezers consume much lower powers compared to conventional optical tweezers. This difference in the system configuration has made the system more adaptable and much less costly than optical tweezers. ${ }^{31}$

\subsubsection{Hydrodynamic Forces}

A microfluidic cell sorting system without any external radiation field or components would seem to be an attractive option for cell manipulation. Using hydrodynamic forces offers this possibility by harnessing the size-dependent differences in diffusion of particles. ${ }^{2,33}$ Microfabrication techniques have been successfully employed to create the microscale features like posts or lattices to harness those differences in particle movements in the channel (Figure 1.9). ${ }^{33}$ Reports on separating blood into its components and using these microstructures to yield particles separation are promising. ${ }^{34}$ These methods, which only utilizes the channel geometry and laminar flow of particles to yield separation, are known as hydrodynamic filtration, pinched flow fractionation (PFF), and asymmetric pinched flow fractionation (AsPFF) (Figure 1.10). Hydrodynamic focusing is realized based on the fact that smaller particles' accessibility to the lowvelocity profile near the inner surface of the channels, while larger particles will stay at regions far from the surface. In a simple form of this technique, particles are separated according to their size 
using sections with different width. ${ }^{35,36}$ Two fluids one containing the particles and the other one without any particles are injected into the channel through separate inlets. The flow containing the particles is pushed towards one side of the constriction by controlling the flow from the other inlet. After the constriction, particle will feel a force which is dependent on their size..$^{35,36}$ Therefore, the difference between the particles positions in the constriction becomes larger after widening of the channel, leading to their separation based on size. Small particles will be deflected towards the wall, while the larger ones will stay in the middle. This technique has been shown to be successfully extended to accommodate more side channels for particle collection. ${ }^{37}$

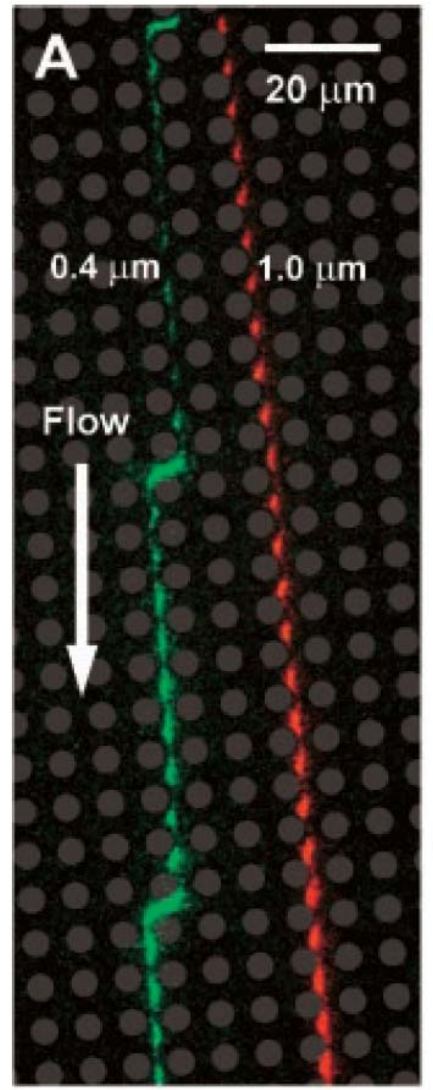

Zigzag Displacement
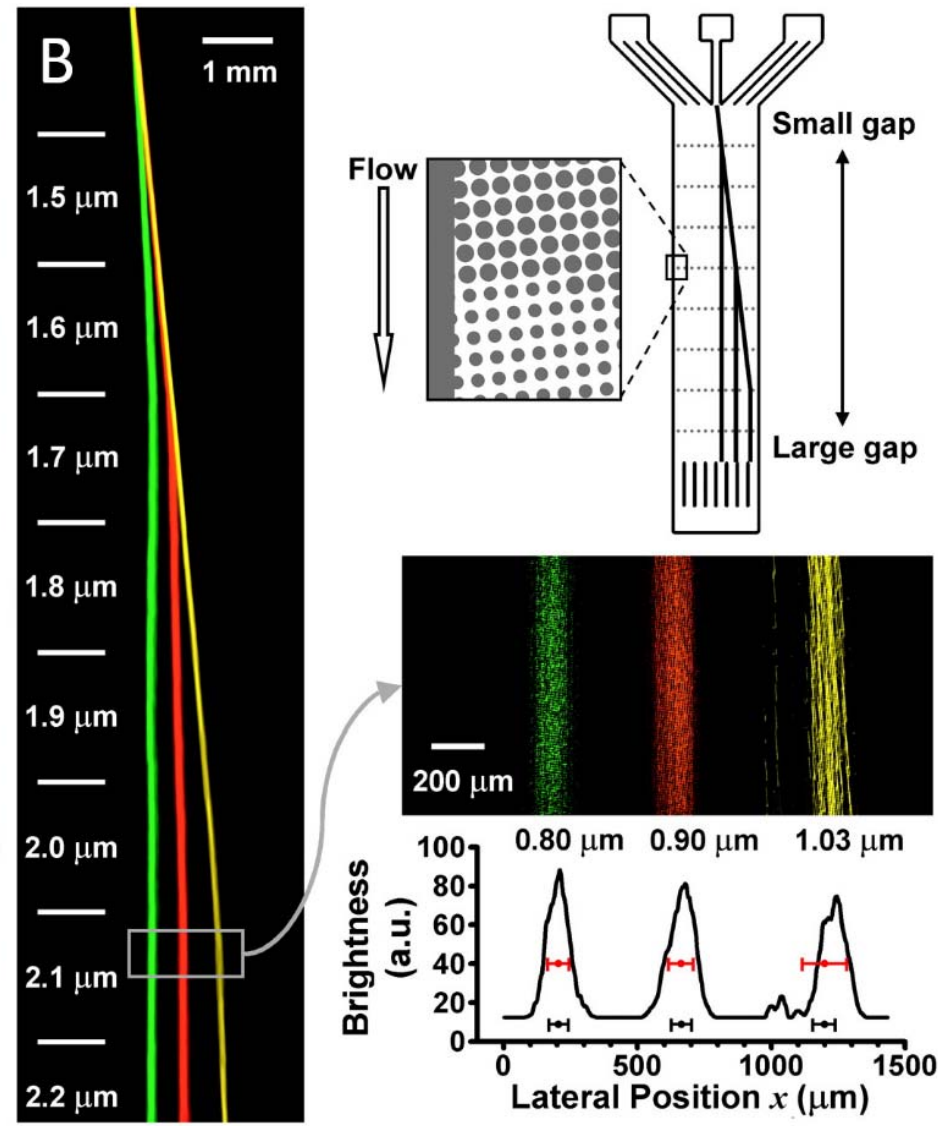

Figure 1.9 Particle separation by deterministic lateral displacement (DLD) method: (a) Fluorescent images of the two transport modes, zigzag and displacement, for polystyrene microspheres. The gray dots are the obstacle matrix, superimposed on the fluorescence image. (b) Fluorescent microspheres with different diameters (green $(0.80 \mu \mathrm{m})$, red $(0.90 \mu \mathrm{m})$, and yellow $(1.03 \mu \mathrm{m})$ ) are separated in a matrix with varying gap size. Changing the obstacle diameters renders a matrix with varying gap sizes, as shown on the left side of the fluorescent picture. The profile on the right side shows the fluorescent intensities of the microspheres bands after separation. (Reprinted with permission from [33]) 

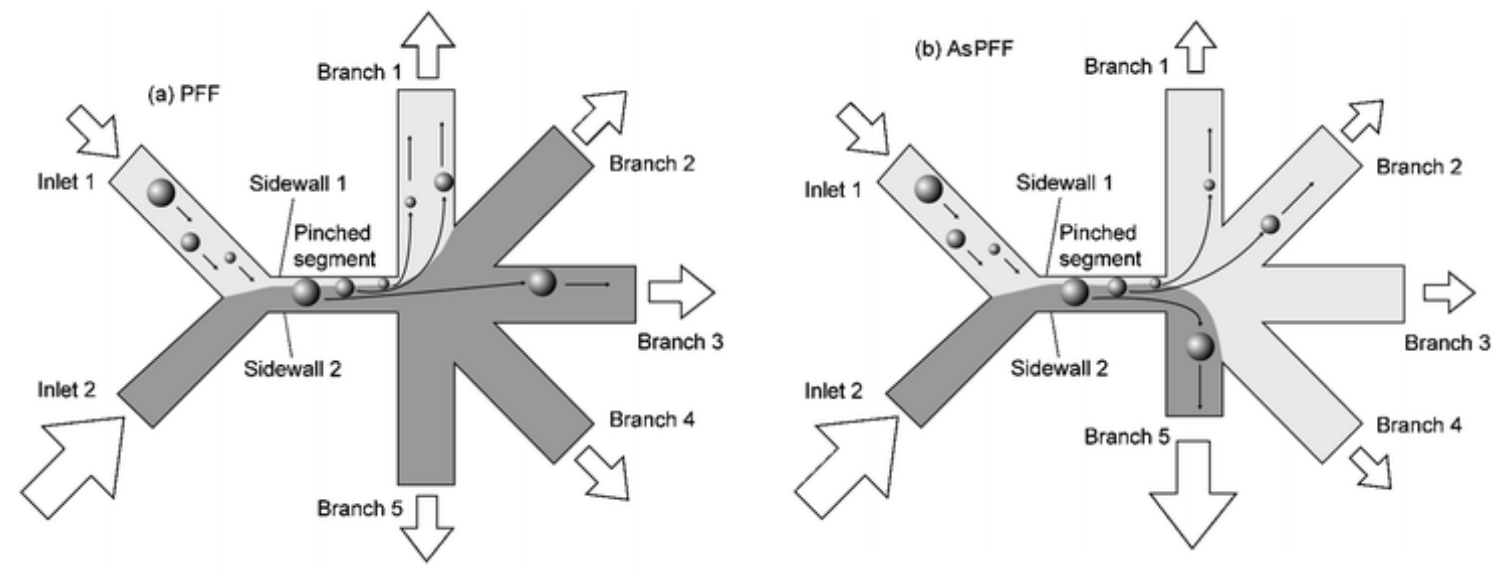

Figure 1.10 Principles of AsPFF and PFF: (a) In PFF, branch channels are identical, and so the flow distributed into them. Thus, Channels 4 and 5 are not used and the difference in the position where particles enter the channels is small. (b) In AsPFF, one channel is designed to be shorter and/or broader compared to the other ones and the flow is distributed asymmetrically, hence the name of the method. Therefore, all the channels are used and the difference in the position where particles enter the channels is large. In both figures, lighter color marks the liquid containing the particles, and the darker one marks the liquid without the particles. The size of the arrows represents the relative magnitude of the corresponding flow rates. (Reprinted with permission from [36])

\subsection{Acoustophoresis}

\subsubsection{Overview}

It has been known for a long time that particles suspended in a fluid phase like a liquid or gas would exhibit different properties if placed within acoustic radiation fields. This is mainly caused by the radiation forces exerted by the field on the particles and by any discontinuity in the acoustic field. As these forces are typically larger in standing waves of ultrasonic frequency compared to progressive waves, ultrasonic standing waves are usually used for particle and cell manipulation applications. ${ }^{38-40}$ The determining factor in how particles are positioned in the field is the geometry of the standing wave, which could be designed to yield either plane, line, or point pressure nodes. ${ }^{2,11}$ In other words, adjusting the field patterns could allow us to agglomerate particles, separate them from the dispensing fluid, wash them from their host fluid to another or fractionate them into different subpopulations. ${ }^{41-43}$ The latter application, as was briefly introduced 
in Acoustic Forces section, is known as acoustophoresis, or the separation through acoustic waves from acousto- "relating to sound" + Greek phorēsis "being carried".

This chapter confines itself to underlining the principles and applications of acousticallyactuated microfluidic systems, also known as acoustofluidic systems, in which acoustic radiation fields have been employed to manipulate and sort particles, with emphasis on the application of the ultrasonic field in cell manipulation and sorting.

\subsubsection{Standing Waves}

A standing wave, first noticed by Michael Faraday in 1831, is a wave each point of which is associated with a constant amplitude. In nature, standing waves form due to either an opposite movement direction of the medium with respect to the wave or because of the interference of the two waves travelling in opposite directions. The most common form of standing waves are created by resonance inside a resonator, where the interference formed between reflected waves from one end of the medium with incident waves from the other end or the source. As this interference creates a pattern in which some points seem to be standing still, the wave pattern formed is called a standing or stationary wave. Standing waves formed by interferences are only generated when the medium is perturbed at specific frequencies known as harmonic frequencies or simply harmonics. Interferences happened at a non-harmonic frequency would lead to irregular or non-repeating disturbances of the medium. The interference of the two waves traveling in opposite directions can be shown mathematically by deriving the equation for the sum of the two waves:

$y(x, t)=A \cos \left(\frac{2 \pi x}{\lambda}+\frac{\phi}{2}\right) \sin \left(\omega t+\frac{\phi}{2}\right)$

where $y(x, t)$ defines the displacement of a point in standing wave with respect to the longitudinal position $x$ at the time $t, A$ is the amplitude of the wave, $\lambda$ is the wavelength, $\omega$ is the angular frequency, and $\phi$ is the phase difference between the two traveling waves. At points in the standing wave which are even multiples of a quarter wavelength, the amplitude is zero and the points are 
called nodes. Locations which are odd multiples of a quarter wavelength are called antinodes and have the maximum amplitude in the standing wave.

\subsubsection{Acoustic Radiation Forces}

The acoustic radiation force exerted on the particles is a gradient of the acoustic potential, described by:

$\boldsymbol{F}^{r a d}=-\nabla U^{r a d}$

where $U^{\text {rad }}$ is the acoustic potential and the particle is assumed to be spherical with a diameter much smaller than the wavelength of the standing wave. For a one-dimensional standing wave of planar type, the resulting acoustic radiation force along the direction $x$ (the transversal direction of the channel in Figure 1.11a) can be then found by differentiation: ${ }^{11}$

$F_{x}^{\mathrm{rad}}=4 \pi \phi(\rho, \beta) k_{x} r^{3} E_{\mathrm{ac}} \sin \left(2 k_{x} x\right)$

in which $\phi$ is the acoustic or acoustophoretic contrast factor, $k_{x}=2 \pi / \lambda$ is the wave vector where $\lambda$ is the wavelength and is equal to $2 w, w$ being the channel width, $r$ is the particle radius, $E_{\text {ac }}$ is the energy density, and $x$ is the distance. ${ }^{11}$

\subsubsection{Acoustic Contrast Factor}

The acoustic contrast factor, as is shown in Eq. 10, is a function of the density and compressibility of both the particles and medium and is given by: ${ }^{11}$

$\phi(\rho, \beta)=\frac{1}{3}\left[\frac{5 \rho_{\mathrm{p}}-2 \rho_{0}}{2 \rho_{\mathrm{p}}+\rho_{0}}-\frac{\beta_{\mathrm{p}}}{\beta_{0}}\right]$

in which $\rho_{\mathrm{p}}$ and $\rho_{0}$ are the density of particle and the medium, respectively, and $\beta_{\mathrm{p}}$ and $\beta_{0}$ are the compressibility of the particle and medium, respectively. Therefore, as can be seen from Eq. 11, the magnitude of the acoustic radiation force experienced by a particle in the chamber is a function of the size of the particle, the energy density, the distance, and the acoustic contrast factor of the particle, which determines how the particle is differentiated from its medium, where positive 
values of $\phi$ afford nodal focusing and negative values afford antinodal focusing of the particle (Figure 1.11b). Therefore, in an acoustic standing wave, particles can be either separated by focusing in one place, i.e. node or antinode, or fractionated based on size taking advantage of the velocity difference, which is a function of the size. ${ }^{11}$

The migration velocity of the particle along the direction $x$ can be also found by the balancing out the acoustic radiation force along the direction $x$ with the Stokes' drag force.

$\sum F_{x}=F_{x}^{\mathrm{rad}}+F_{x}^{\mathrm{drag}}=0 \Rightarrow v_{x}=\frac{2 k_{x} r^{2} E_{\mathrm{ac}}}{3 \mu} \phi(\rho, \beta) \sin \left(2 k_{x} x\right)$

Eq. 12 shows that the velocity of the particle scales with the second power of the radius. Therefore, size-based fractionation in an acoustic standing wave is mainly realized through the difference in the velocity of the particles of different size.

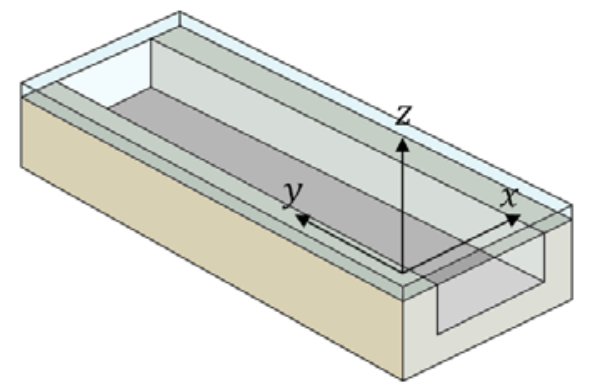

a)

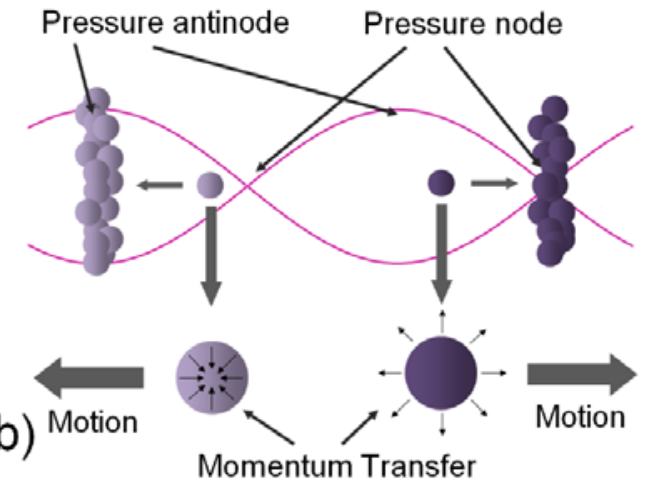

Figure 1.11 Schematic of a) a typical acoustic perturbation device, in which $y$ marks the transversal direction of the channel and $x$ represents the flow direction. b) An overlaid ultrasonic standing halfwave, its radiation force, and the positions of the node and antinode in the standing wave. Black arrows denote the direction of the momentum transfer and grey arrows denote the direction of the net force and thus the resulting motion.

\subsubsection{Acoustic Particle Manipulation}

As mentioned earlier, acoustic radiation fields, particularly those generated through ultrasonic standing waves, offer the possibility of manipulating and separating particles suspended in fluid media. ${ }^{2}$ The magnitude of the radiation force depends on the distance of the particle from the pressure node, in the case of a nodal focusing. ${ }^{11,44}$ Given the fact that microfluidic systems have 
active sorting regions in the micron size regime, the wavelength of the standing wave is to be chosen in the same size scale to allow the manipulation of the particles. In other words, for a microfluidic system with an active sorting region of a few hundred microns wide, ultrasonic frequencies of a few megahertz should be used to manipulate the particles. For instance, Figure 1.12a shows a schematic view of particles randomly dispersed in a fluid system sandwiched between a transducer layer and a reflective layer. ${ }^{38}$ Upon actuation of the transducer at the half-wavelength resonance mode, a standing wave will be established inside the container applying a pressure field with maximum and minimum points of pressure. Figure $1.12 \mathrm{~b}$ shows the direction and relative magnitude of the acoustic radiation force in the fluid container with blue arrows showing the direction of the net radiation force and blue curve showing the relative magnitude of the applied force. ${ }^{2}$ The purple curves show the pressure profile of the standing wave yielding one node in the middle of the channel and two antinodes at the walls. Particles which are denser and less compressible than their suspending medium, would aggregate at the nodal regions as they exhibit a positive acoustic contrast factor. (Figure 1.12c).
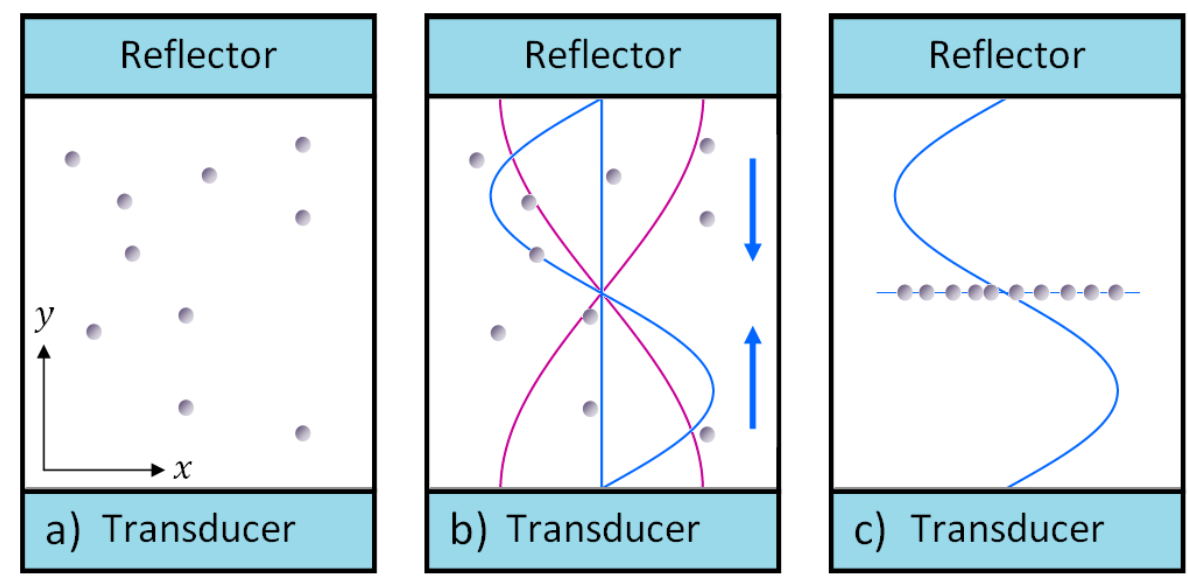

Figure 1.12 Particle manipulation using ultrasonic standing waves: (a) Particles are randomly dispersed before actuation of the channel. (b) Actuation of the channel at a half-wave length resonance mode, causing the formation of a pressure node at the center line of the fluid layer shown in purple and therefore a net force in the direction of the arrows is formed in line with the blue line. (c) The established radiation force causes the movement of the particles towards the center node. (Reproduced with permission from [2]) 
The ability to differentiate or isolate particles based on their physical properties make acoustic standing waves suitable for filtration or concentration applications. ${ }^{2,45}$ Figure 1.13 shows a schematic illustration of a particle concentration system which is known as a particle agglomeration device. In this device, first acoustic radiation forces are employed to focus the particles initially suspended in a medium (Figure 1.13a) to the nodal or antinodal regions of the wave (Figure 1.13b) and then as particles become more aggregated they are forced to sediment as a result of either the gravitational forces exerted on the agglomerate or removing the radiation field (Figure $1.13 \mathrm{c})^{2}$
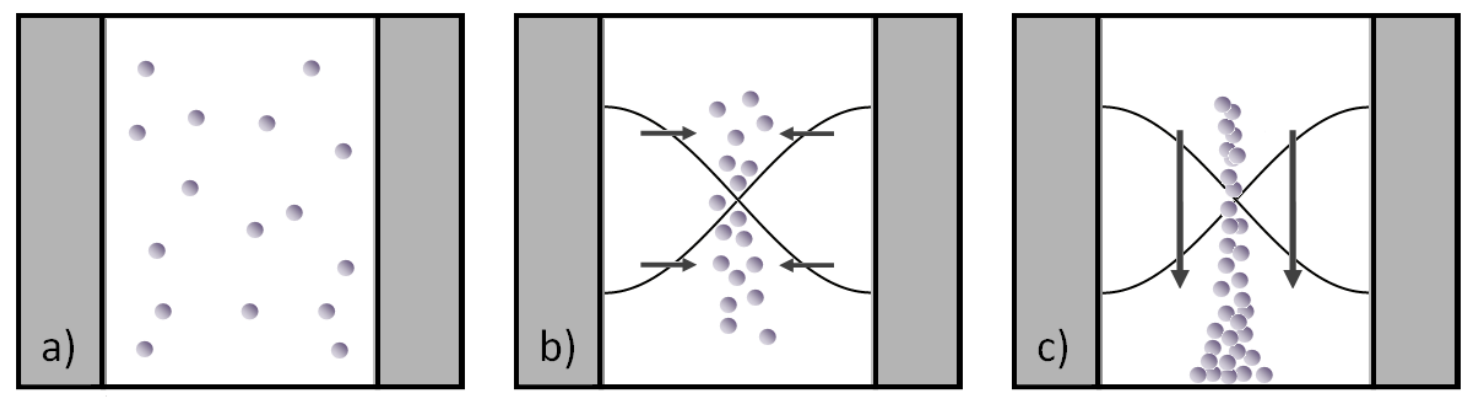

Figure 1.13 Schematic of an acoustic sedimentation system: (a) Particles are randomly suspended in the chamber before actuation of the acoustic transducer. (b) Same particles in a) start to migrate towards the nodal plane of the chamber upon actuation of the acoustic transducer. (c) As the agglomeration grows, the particles clump tends to sediment under gravity. (Reproduced with permission from [2])

Although the device in Figure 1.13 can only be used for batch processing, with some modification in the architecture of the device it is possible to use the same device as a continuous system. Figure 1.14 shows a schematic illustration of such a device, which is a wet-etched silicon microchannel containing one inlet and three outlets. ${ }^{46}$ In this device, the fluid containing the particles is continuously injected into the chamber of the device and as the acoustic standing wave is set up in the chamber the particles are focused into the nodal region and collected from the middle outlet, while the fluid is directed towards the upper and lower outlets. ${ }^{46}$ What makes the particles focused in the middle after the separation in the radiation field is the laminar nature of the flow inside a microfluidic device like what is shown in Figure 1.14. 


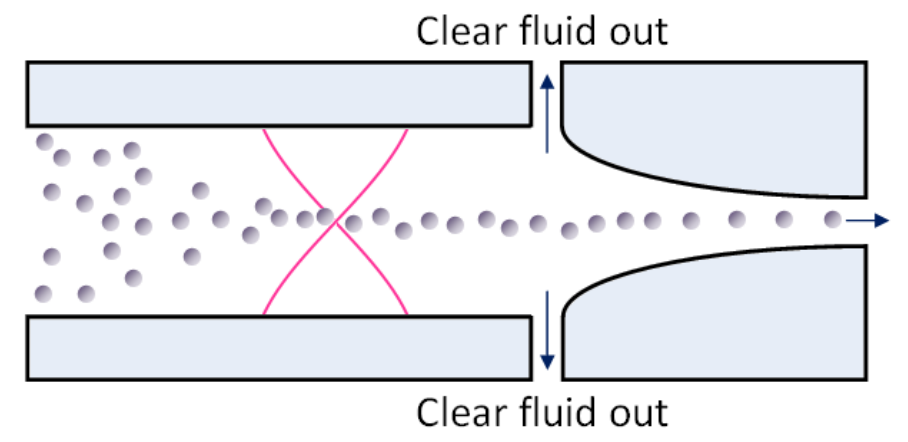

Figure 1.14 A continuous microfluidic system for the acoustic separation of particles from their suspending medium. Particles after separation are stayed focused in the middle of the channel until they are collected from the middle outlet. Clear fluid is separated via two side outlets. (Reproduced with permission from [46])

Another application of acoustic radiation fields is in washing particles or cells form one fluid to another, which is a very important characteristic of many analytical studies. Devices used for such application rely on the fact that in a microfluidic channel two fluids could flow in contact with each other with minimal mixing due to the laminar nature of the flow in such devices. ${ }^{47}$ Figure 1.15 shows an illustration of a device used for washing particles from one fluid to another. As can be seen, fluids entering the channel form two separate inlets remain unmixed throughout the channel and finally directed towards two outlets. What makes particles migrate from one medium to the other is the effect of acoustic radiation force on the particles which concentrates the particles towards the node in the second medium. ${ }^{47}$

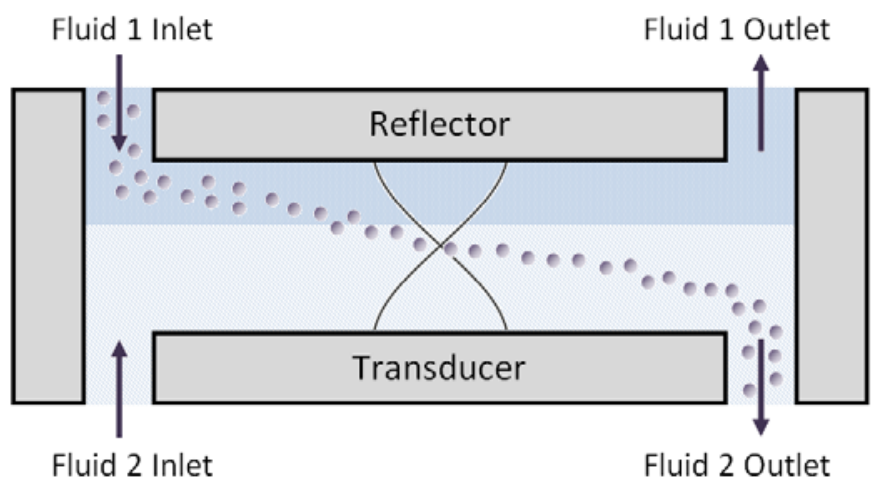

Figure 1.15 Acoustic washing of particles. Under laminar flow conditions, particles from one medium can be washed into the second medium through establishing an ultrasonic standing wave in the chamber forcing particles to migrate towards the second medium. (Reproduced with permission from [47]) 
Standing acoustic waves have been also used to enhance the performance of biosensor in biomedical assays. ${ }^{2,48}$ Figure 1.16 shows a schematic illustration of a device in which an ultrasonic standing wave is established to force the particles of interest towards a biosensor surface mounted on a reflector layer. ${ }^{49}$ In this device, the ultrasonic standing wave is set up such that the pressure node is established on the surface of the biosensor layer. In other words, in contrast to the halfwavelength resonating devices which have one node in the center of the channel and two antinodes at the walls, the device described here would have a quarter-wavelength configuration with one node on the biosensor layer on the reflector wall and one antinode at the transducer wall. Using a device like what is shown in Figure 1.16, the concentration of Bacillus subtilis var. niger spores on a surface coated with an antibody, shown as the biosensor surface in the figure, has been demonstrated to be enhanced 70-fold compared to the conventional counterpart. A system like this has the potential to substantially enhance the sensitivity of the biosensors, especially in microfluidic systems suffering from the diffusion limit of the particles.

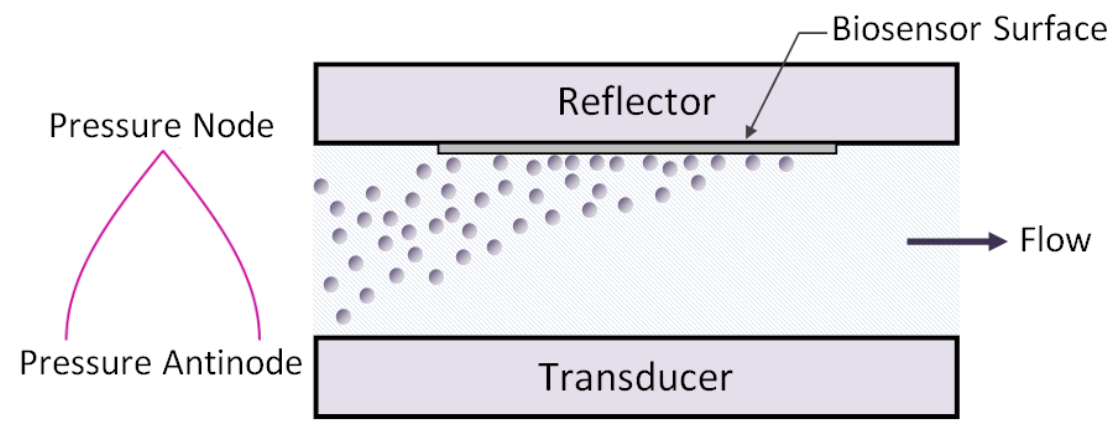

Figure 1.16 Enhanced capture of bacteria on biosensor surface via acoustic field. An overlaid quarterwavelength standing wave with a half-wavelength thick reflector would enhance the capture of Bacillus subtilis var. niger spores on the surface of the immunosensor surface. (Reproduced with permission from [49])

As was demonstrated mathematically in Section 1.2.3, the acoustic radiation force exerted on a spherical particle scales with the cubic power of its radius. Therefore, particles of different size but of same type would experience different magnitudes of radiation force which render them different lateral velocities. This feature of acoustic fields can be harnessed to yield size fractionation 
in microfluidic systems if the laminar flow is stabilized in the channel and the residence time is chosen properly to avoid focusing all the particles in the nodal region. In a device build based on the this attribute, particles of different size are separated in a quarter-wavelength channel with a laminar flow as they experience different magnitudes of acoustic radiation force.

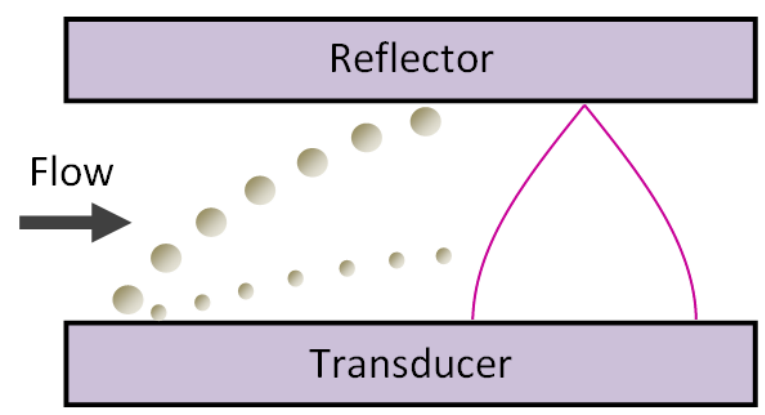

Figure 1.17 Size-selective acoustofluidic separation. Particles are fractionated into two or more groups in a quarter-wavelength channel as they experience different magnitudes of acoustic radiation force. The particles are often prefocused before entering the radiation field. (Reproduced with permission from [2])

\subsubsection{Acoustophoresis of Biological Particles}

\subsubsection{Cell Acoustophoresis}

The attributes that make acoustophoresis a desirable method for particle separation applications have been widely reported..$^{50}$ Other than being purely dependent on the physical properties of the particles and the medium, its high-throughput screening capability and gentle action on the particles provide most of its development impetus. ${ }^{51}$ It is particularly the latter that attracts attention in the field of cell separation. ${ }^{52} \mathrm{~A}$ handful of studies, ${ }^{53,54}$ indeed, have succeeded in making it to the point of being considered as practical separation methods; but, if anything, they have at the same time highlighted the challenges facing the further development of this method. One constraint in particular has directed further functionality in the separation applications: different cell types have been reported to behave similarly, in that in all of the studies the cells would tend to migrate toward the high-pressure zone(s) or node(s). ${ }^{55}$ In other words, for cells of 
the same size any difference in the rigidity of cells would appear to be ineffective to make them differentiable in the acoustic radiation field. Nevertheless, acoustically-driven devices are increasingly suggested as gentle, high-throughput technologies capable of handling a wide variety of applications involving cells or other types of bioparticles like viruses, bacteria, extracellular vesicles, etc.

This chapter confines itself to underlining some of the recent applications of acousticallyactuated microfluidic systems in manipulating and sorting cells and other bioparticles, with emphasis on the acoustophoresis of cells.

As mentioned earlier, one of the application of acoustic standing waves is in transferring particles and cells from one liquid medium to another, which is an important step in many bioanalytical investigations. Analogous to the device described in Section 1.2.5, Hawkes et al. ${ }^{56}$ developed a method for washing yeast cells from one medium to another, making use of laminar flow and a continuous FFF. In their device, a half-wavelength standing wave is designed in the center line of the channel fitted with two inlets and two outlets. The suspension containing the yeasts remains in contact with the yeas-free liquid without mixing, provided that the laminar nature of the flow is maintained in the device. ${ }^{56}$ Upon actuation of the device, cells promptly begin positioning to the nodal plane of the wave located in the other fluid.

Tenje et al. ${ }^{57}$ developed a device, based on the same concept, to laterally wash plasma proteins and other low-molecular-weight compounds from red blood cell (RBC) concentrates into a buffer solution (Figure 1.18). Preparation of protein-free RBC concentrate is particularly important for blood transfusion for patients with Immunoglobin A (IgA) deficiency, a common primary immunodeficiency diseases characterized by undetectable levels of $\operatorname{IgA}$ in the blood. The application of acoustophoresis for the preparation of protein-free RBC concentrate offers higher efficiencies than the conventional dilution and centrifugation methods. 


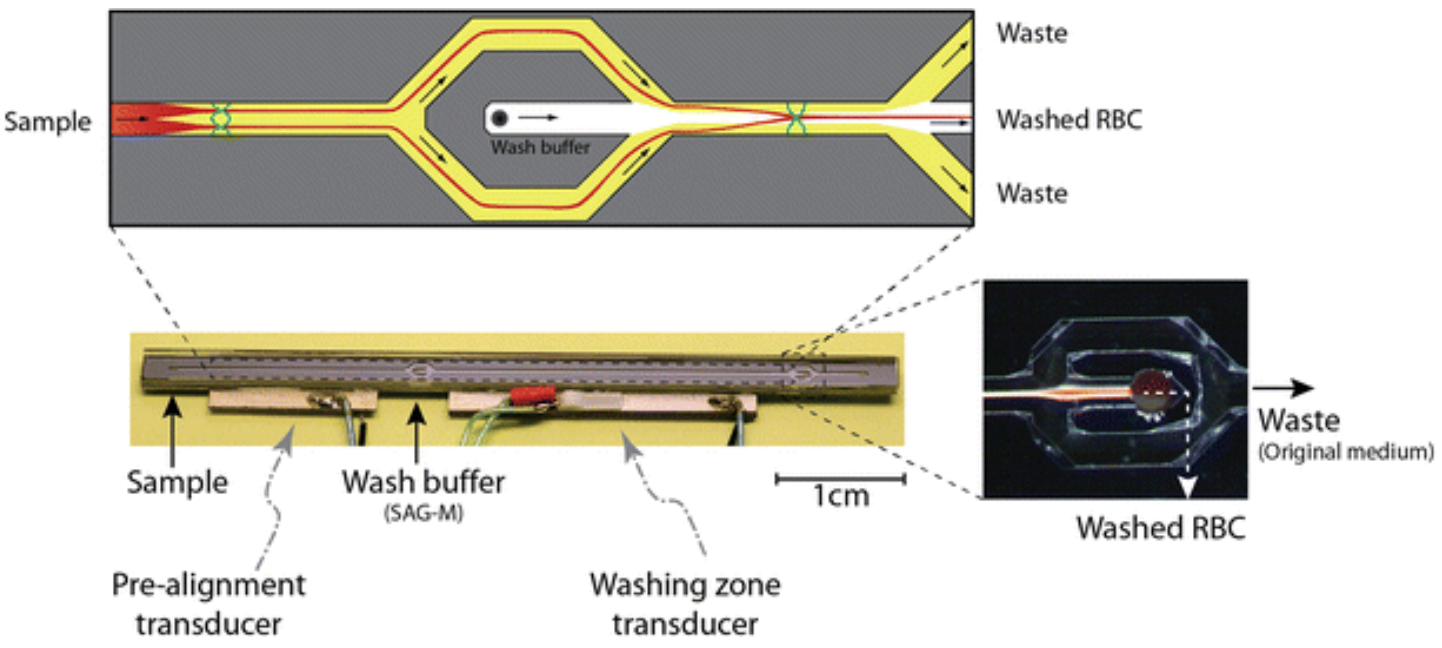

Figure 1.18 Acoustophoretic removal of proteins from RBC concentrates. The micrograph on the right shows the RBCs cleared from proteins drawn off from the center outlet and the original medium from the side outlets. (Reprinted with permission from [57])

More recently Antfolk et al. ${ }^{58}$ developed an acoustophoretic chip for the separation of circulating tumor cells (CTCs) from white blood cells (WBCs). In this work, A blood sample spiked with prostate cancer cells (DU145) was continuously injected into a microfluidic device fitted with two piezoelectric transducers for the pre-alignment and separation of cells. High recovery rates were achieved in this work which were found to rise to even higher values upon increasing the acoustic radiation intensity. Unlike multi-laminar stream devices used for washing particles and protein removal from blood samples, the device used in this study has only one inlet, decreasing the risk of accuracy loss occurred due to the mismatch of acoustic properties in the channel. Furthermore, while cells are often diluted in devices utilizing hydrodynamic focusing, they can be concentrated after recovery in this one-inlet configuration. ${ }^{58}$

The ability to detect and isolate CTCs from the blood making use of only the inherent properties of cells is of high importance, as CTCs may undergo epithelial-mesenchymal transition (EMT), during metastasis, upon which many characteristics of epithelial cells are lost, ${ }^{59}$ posing a challenge for the conventional separation methods relaying on epithelial cell biomarkers. ${ }^{58}$

In a seminal work, Li et al. ${ }^{60}$ successfully separated CTCs from the peripheral blood of patients with breast cancer, using an acoustophoretic device with tilted-angle standing surface 
acoustic waves (taSSAW). In this work, the functionality of the device has been first validated by separating cultured human breast cancer (MCF-7) and cervical cancer (HeLa) cells from WBCs. In the next step, the device was shown to successfully perform a label-free separation of rare breast CTCs from WBCs in their viable state (Figure 1.19).

The principle of taSSAW was introduced by the same group in an earlier work ${ }^{61}$, in which a numerically optimized configuration of tilted standing waves was shown to offer higher efficiency and sensitivity compared to the conventional acoustophoretic platforms. ${ }^{61}$ The efficiencies obtained for the size-based separation of polystyrene microbeads by that device were as high as $99 \%{ }^{61}$ Furthermore, taSSAW was shown to be capable of differentiating based on the compressibility for particles and cells of the same size and density. ${ }^{61}$ That device was also successful in label-free isolation of human breast cancer (MCF-7) cells from WBCs, demonstrating the functionality of the device for biomedically-relevant applications.

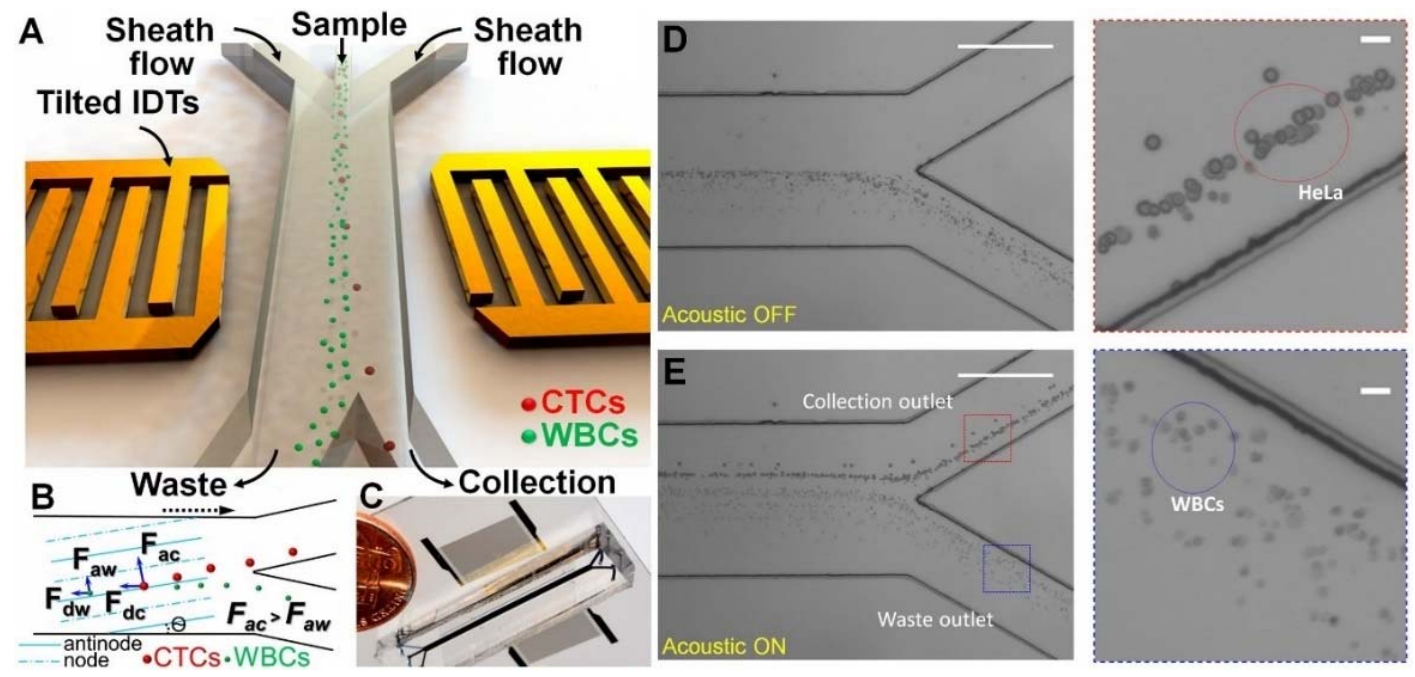

Figure 1.19 a) Schematic illustration of the taSSAW device. b) In taSSAW-based cell separation, nodes and antinodes are designed to locate at an angle of inclination $(\theta)$ with respect to the flow. CTCs which are larger in size compared to WBCs experience a greater acoustic force, causing them to move in a greater distance perpendicular to the flow direction. The other force experienced by the cells is the drag force, which is again greater for the CTCs. c) Actual image of the taSSAW device. d) Mixture of HeLa cells and WBCs in the channel with the acoustic off. All the cells are driven along the lower side channel, exiting from the same outlet. e) Upon actuation of the device, HeLa cells which are larger are pushed toward the upper side channel, whereas the WBCs remain in the lower stream line, leading to the separation of the two cell types. The two zoomed-in images are time-lapsed micrographs of the outlets. (Reprinted with permission from [60]) 
The main observation emerging from the reviewed studies is that cell isolation and separation using acoustophoresis has been restricted mainly to size contrast. This mainly stems from the fact that cells often do not vary much in density to yield a change in the acoustic contrast factor enough to use in a separation. Wyatt Shields et al., ${ }^{62}$ however, devised a method to overcome this issue by binding cells with elastomeric particles exhibiting a negative acoustic contrast factor. ${ }^{62}$ This "particle-based" acoustophoretic method made use of this unique attribute of silicone particles to yield separation between the cells which were specifically immunolabeled with those particles and the unlabeled cells which would migrate to the node. ${ }^{62}$ Figure 1.20 shows how elastomeric particles functionalized with streptavidin (SA) bind to acute myelogenous leukemia (KG-1a) cells and transport them to the antinodes of the acoustic standing wave. ${ }^{62}$
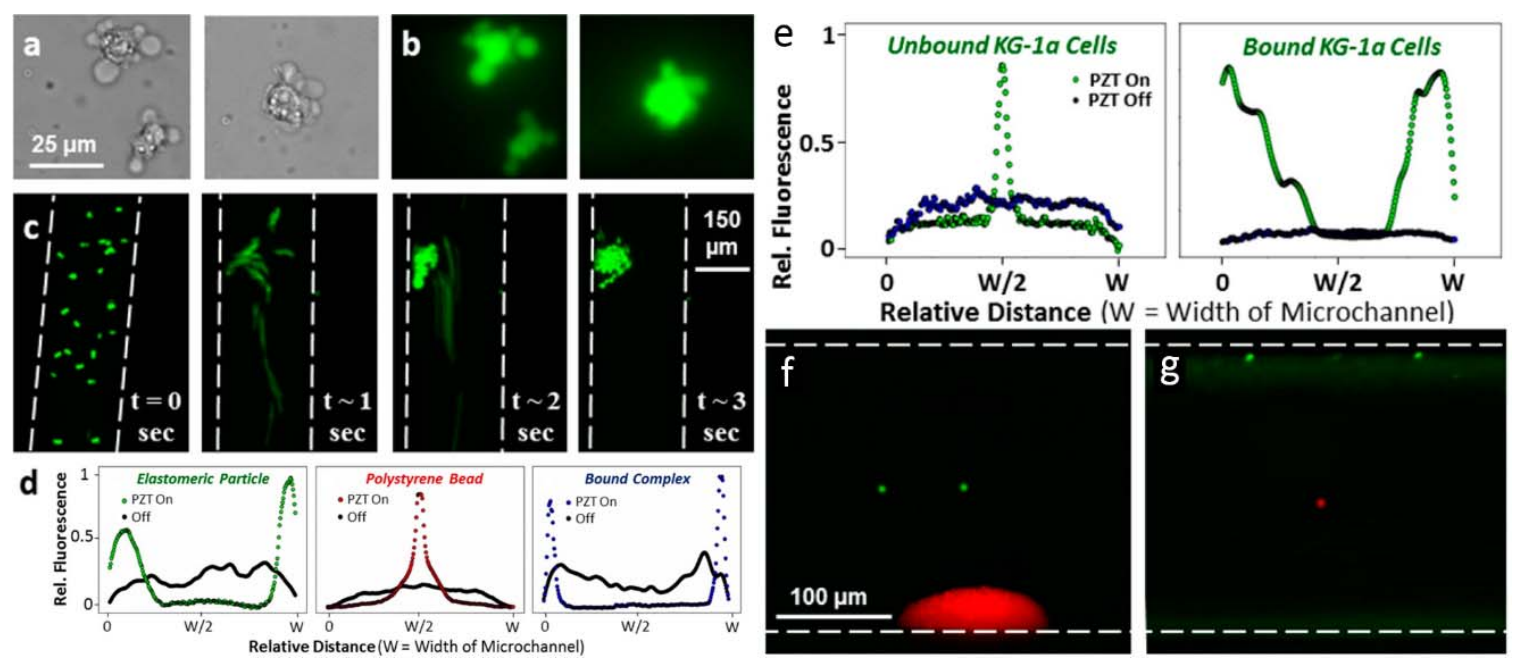

Figure 1.20 Principle of particle-binding cell acoustophoresis using elastomeric particles: a) Brightfield and b) fluorescent images of SA-adsorbed elastomeric particles bound to green-stained KG-1a cells. c) Complexes of the cells and elastomeric particles migrating to the antinodes when the acoustic transducer is on. d) Fluorescence intensity distributions of SA-adsorbed elastomeric particles (left), biotinylated polystyrene microbeads (middle), and complexes of particles (right) across the microchannel width $(\mathrm{W})$. e) Fluorescence intensity profiles showing the separation of the unbound cells (left) from the bound cells (right). Fluorescent images of f) green KG-1a cells and the elastomeric particles embedded with Nile red focusing at the node and antinodes, respectively, and g) SA-adsorbed elastomeric particles bound to green, anti-CD34-biotin-labeled cells are separated from red-stained cells without anti-CD34-biotin by migrating to the antinodes and nodes, respectively. (Reprinted with permission from [62]) 


\subsection{Lipid Bilayers}

\subsubsection{Overview}

The lipid bilayer membrane is the common underlying structure in living systems that confines the cytoplasm and cellular organelles inside the cells, thereby maintaining the integrity of cells and establishing the essential difference between the interior and outside environment. ${ }^{63,64}$ Biological membranes act as selective barriers, i.e. they are impermeable to most water-soluble molecules, and provides a medium for communication with the extracellular environment. ${ }^{65}$ Therefore, the membrane plays a crucial role in many cellular processes that are associated with transport, signaling, recognition, and immunological and enzymatic activities. ${ }^{66}$ In response to these requirements, membranes have become increasingly complex in terms of composition, structure and organization. ${ }^{67}$ Nonetheless, all biological membranes are formed via self-assembly of lipid molecules which are held together by noncovalent interactions. The plasma membrane, also known as the cytoplasmic membrane, which is composed of mainly lipids ( $\sim 50 \mathrm{wt} . \%)$, proteins ( 40 wt.\%), and carbohydrates ( $\sim 10$ wt.\%), is often made up of three distinct layers: 1$)$ a lipid bilayer membrane in the middle, embedded with different biomolecules, 2) a glycoproteinpolysaccharide covering, extended towards the extracellular space, and 3) a network of interlinking filaments and tubules, known as cytoskeleton, extended towards the intracellular environment. ${ }^{67}$

Cells are covered by a protective layer from outside which is formed by the carbohydrates bound to the tails of either the glycolipids or glycoproteins imbedded on top of the lipid bilayer. Known as glycocalyx, this protective layer plays an important role in cell adhesion and recognition processes. ${ }^{67}$ To the inner side of the membrane is attached the cytoskeleton, which is a complex network of proteic filaments. This dynamic network in addition to giving cell its shape is responsible for the cellular motility and motions of the organelles inside the cell. The lipid bilayer is barrier between the interior and extracellular environment is mostly composed of lipids in between which various types of proteins are embedded. ${ }^{68}$ Many specific cellular functions are 
modified by these proteins. Lipids are amphiphilic molecules, composed of a hydrophobic tail region and a hydrophilic headgroup. There exist a great number of lipids in biological membranes, differing to one another in the length and degree of saturation of the tail and in the type of head group. Lipid fatty acid tails containing 14 to 24 carbons, with usually one saturated chain and one mono- or polyunsaturated chain are more common. Glycerophospholipids or phosphoglycerides are one of the main classes of membrane lipids with a glycerol backbone to which two fatty acid chains and a headgroup containing a phosphate molecule bound to either one of the ethanolamine, choline, serine, glycerol, or inositol molecules. ${ }^{69,70}$ The lipids with these headgroups are known as phosphatidylethanolamines (PtdEtns or PEs), phosphatidylcholines (PtdChos or simply PCs), phosphatidylserines (PtdSers or PSs), phosphatidylglycerols (PtdGros or simply PGs), and phosphatidylinositols (PtdInss or PIs), respectively. ${ }^{69}$ Glycerophospholipids along with sphingomyelin which has a different backbone are collectively known as phospholipids which, when placed in water, self-assemble to form bilayers in the size range of around $5 \mathrm{~nm}$. The other major group of lipids are glycolipids which, along with a carbohydrate attached to it, extend from the phospholipid bilayer into the extracellular environment where they act as a recognition site for other molecules or a place for cells to attach to other cells.$^{67}$ Phospholipids, glycolipids, and sterols, despite variations in their contents, are the three major lipids found in almost all biological membranes. ${ }^{63,71}$

Our current perception of membrane structure can be traced back to an important discovery in 1925 by Gorter and Grendel ${ }^{72}$ that cellular membranes are composed of two monolayers. Ten years later, Danielli and Davson ${ }^{73}$ extended this finding by describing the bilayer configuration as a sandwich of lipids incorporated on both sides by proteins. In 1957, Robertson ${ }^{74}$ proposed his nowfamous unit membrane model which characterizes the membrane as a trilaminar pattern consisting of a lipid leaflet sandwiched between two layers of proteins. most well-known description of cellular membranes, namely the mosaic fluid model, was however devised by Singer and 
Nicolson $^{75}$ in 1972 . Shows a 2D and a 3D illustration of our modern-day perception of membrane model, demonstrating the lateral heterogeneity and lipid domains on the membrane. ${ }^{76}$
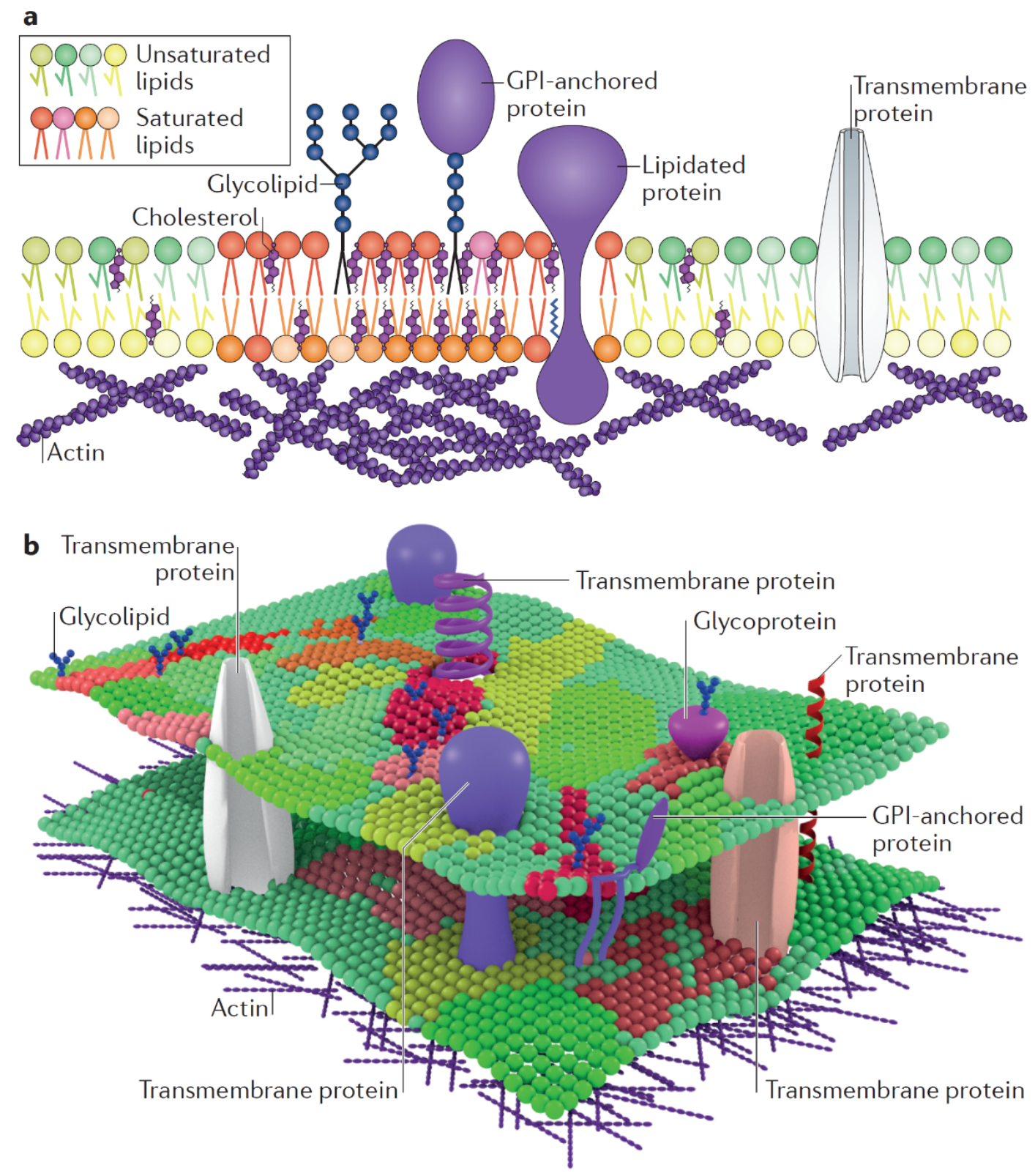

Figure 1.21 Schematic illustration of a biological membrane, showing a), the saturated and unsaturated lipid molecules, cholesterol, the actin network, glycolipids, glycosylphosphatidylinositol (GPI)anchored proteins, lipidated proteins, and transmembrane proteins. The subdomains enriched in saturated lipids, cholesterol, (GPI)-anchored and lipidated proteins are known as lipid rafts, characterized by their higher packing order and decreased fluidity compared to the non-raft domains. b) Uneven component distribution in each leaflet and among the inner and outer leaflets of a biological membrane, leading to a membrane organization consisting of various small raft-like and non-raft domains. (Reprinted with permission from [76]) 


\subsubsection{Membrane Models in Biology}

Two model systems, supported membranes and vesicles, have been historically used to study the biophysical properties of biological membranes. ${ }^{67}$ A supported membrane, commonly known as supported lipid bilayer (SLB), is a model system consisting of one or several lipid bilayers

placed on top of a polished glass or mica surface. ${ }^{77,78}$ Vesicles are the second group of model systems, which if made of lipid bilayers are also called liposomes. Depending on their size, vesicles with one lipid bilayer are categorized into small unilamellar vesicles (SUVs), large (LUVs) and giant unilamellar vesicles (GUVs). Large and giant vesicles with more than one concentric bilayers are called large multilamellar vesicles (MLVs) and giant multilamellar vesicles (GMVs). Among various types of vesicles, giant unilamellar vesicles (GUVs) are particularly of interest for biophysicists as their diameter size $(1-100 \mu \mathrm{m})$, the size range of most biological cells, and unilamellarity make them perfect candidates to serve as model systems in mimicking the behavior of real cells. ${ }^{79,80}$ GUVs were first proposed as model systems by Reeves and Dowben ${ }^{81}$ in 1969. Publications that reports the application of GUVs in biophysical characterizations of membranes have substantially increased over the last 20 years, demonstrating that GUVs have become wellstablished model systems for many biophysical and biochemical investigations. ${ }^{82}$ The main benefits to be gained from using GUVs is the easiness of visualization, size scale similar to that of many cells, and the good control over molecular composition.

\subsubsection{Elastic Deformations of Lipid Bilayers}

Figure 1.22 shows the schematic illustration of four elastic deformations of biological membranes that are typically used to model the membranes and the proteins imbedded in them. These four elastic deformations are: the area expansion or stretching, bending, thickness change, and shearing. Of these deformations, bending is probably the most commonly studied one, the membrane response to which is characterized by either the mean curvature elastic modulus, also referred to as the bending rigidity $\left(k_{c}\right)$, or the Gaussian curvature elastic modulus $\left(\bar{k}_{c}\right){ }^{67}$ 


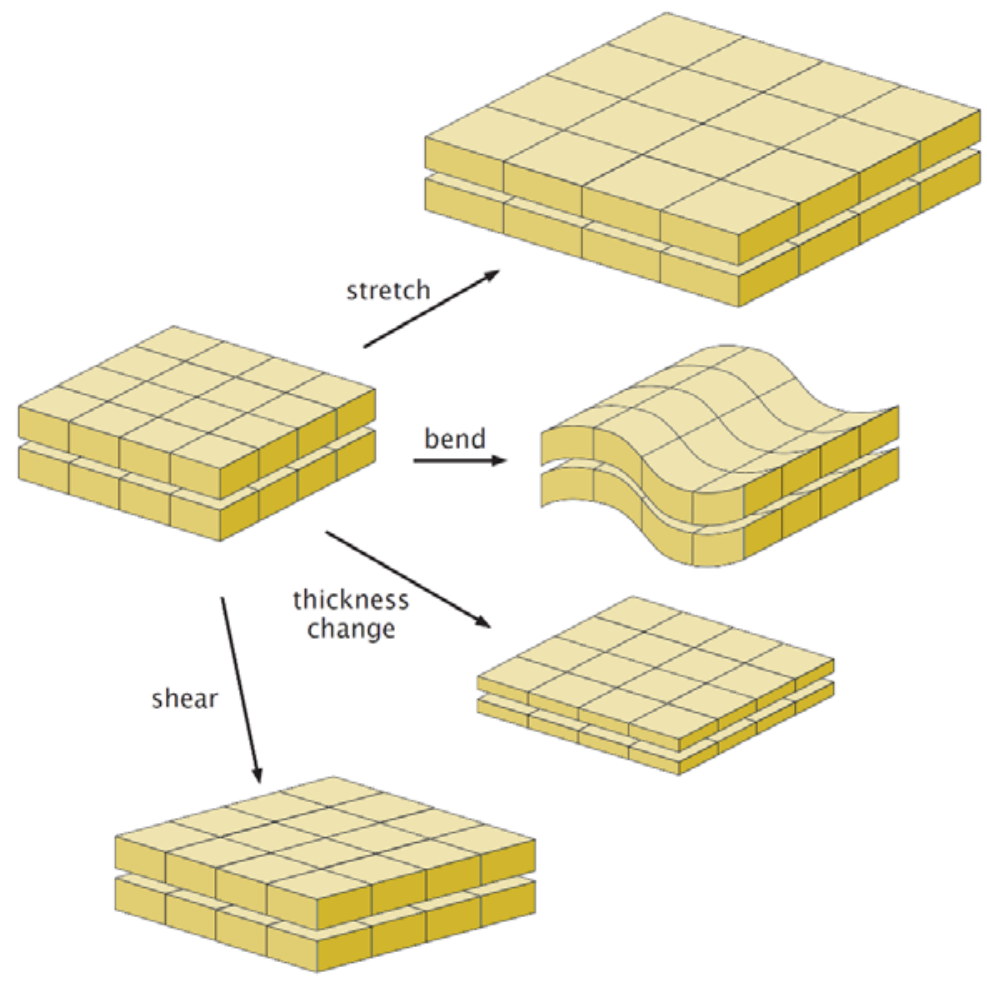

Figure 1.22 Schematic representation of four different modes of bilayer deformations: the stretching or expansion of the membrane, bending, thickness change, and shearing of the membrane. (Reprinted with permission from [63])

The mean curvature elastic modulus, i.e. the $k_{c}$, is defined as the change in the mean bending moment per unit length of the bilayer, $\bar{M}=k_{c} \bar{c}$, with respect to the mean curvature: ${ }^{69}$

$k_{c}=\left(\frac{\partial \bar{M}}{\partial \bar{c}}\right)_{A, \bar{c}_{G}}$

where the mean curvature, $\bar{c}$, is the sum of the principal curvatures $\bar{c}=c_{1}+c_{2}$, with the principal curvature axes being the inverse of the principal radii of curvature, $R_{1}$ and $R_{2}$, describing by $c_{1}=$ $1 / R_{1}$ and $c_{2}=1 / R_{2} \cdot{ }^{69}$

The Gaussian curvature elastic modulus is defined as the change in the Gaussian bending moment per unit length of the bilayer, $\bar{M}_{G}=\bar{k}_{c} \bar{c}_{G}$, with respect to the Gaussian curvature: ${ }^{69}$

$\bar{k}_{c}=\left(\frac{\partial \bar{M}_{G}}{\partial \bar{c}_{G}}\right)_{A, \bar{c}}$ 
where the Gaussian curvature is $\bar{c}_{G}=c_{1} c_{2}$. The total curvature elastic energy would be then: ${ }^{69}$

$\Delta G_{c}\left(\bar{c}, \bar{c}_{G}\right)=\frac{1}{2} k_{c} A\left(\bar{c}-c_{0}\right)^{2}+\bar{k}_{c} A \bar{c}_{G}^{2}$

in which $c_{0}$ is the intrinsic curvature of the membrane. $k_{c}$ or the bending rigidity of the membrane has been measured mostly through the analysis of thermal fluctuations of the membrane ${ }^{83,84}$ or by micropipette aspiration studies conducted on giant vesicles. ${ }^{85}$ More recently, the analyses of the effects of curvature fluctuations on the XRD patterns obtained from multilamellar lipid layers have been used to estimate the $k_{c}$ of membrane. ${ }^{86}$

\subsubsection{Effect of Molecular Structure}

The molecular structure of the lipids constituting a membrane strongly affect the bending stiffness of the membrane. In this regard, the $k_{c}$ of membranes have been shown to scale with the square of the thickness of the hydrophobic region of the chain:

$k_{c} \sim \frac{d_{h}{ }^{2}}{a}$

where $d_{h}$ is the thickness of the hydrophobic region of the chain and $a$ is the area per molecule of the lipids in the membrane, which is assumed to be constant. Figure 1.23 illustrates this strong dependence for three different lipids, namely DLPC, DMPC, and DPPC.

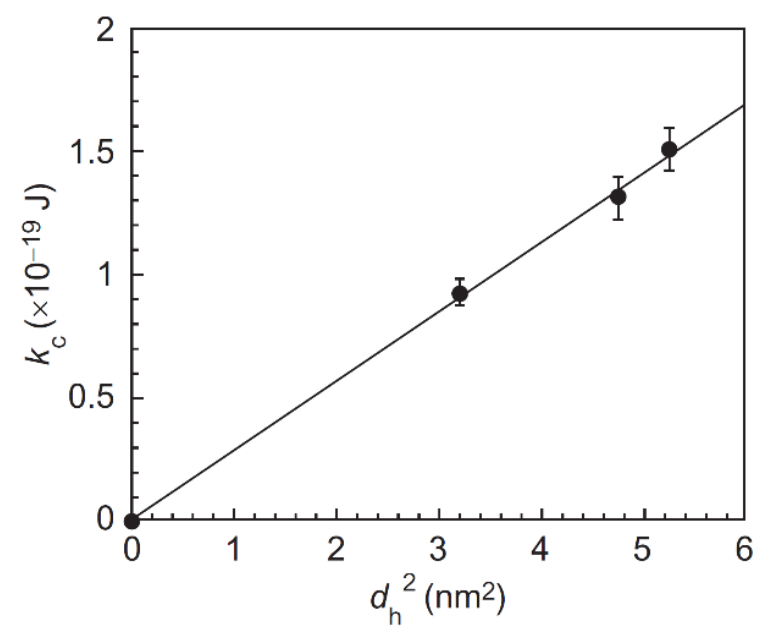

Figure 1.23 Relationship between the $k_{c}$ for DLPC, DMPC, and DPPC, and the thickness of the hydrophobic region of the lipids, $d_{h}$. (Reprinted with permission from [87]) 
It has also been shown that the $k_{c}$ of membranes composed of saturated or monounsaturated PCs scales with the chain length. ${ }^{88}$ Moreover, it has been observed that bilayers composed of lipids with two or more alternating cis double bonds on one or both chains of the lipid are more compressible than the ones composed of saturated or monounsaturated lipids. ${ }^{88}$

\subsubsection{Effect of Temperature}

The temperature-induced changes in the mechanical properties of membranes form the basis upon which this work has been founded. The changes in the $k_{c}$ of the membrane, in particular, also known as the thermoelastic properties of membrane, has been the subject of numerous studies. ${ }^{67}$ The dependency of the $k_{c}$ on the temperature has been summarized as a sharp drop close to the main phase transition temperature and an increase after the main transition, followed by a small decrease, ending with a stabilization around a few degrees after the main transition. The sudden drop close to the main phase transition temperature, is accompanied by anomalies, which render the membranes softer. ${ }^{89-91}$

\subsubsection{Incorporation of Other Molecules}

As mentioned earlier, naturally-occurring membranes host a large number of components that can affect the physical properties of the membrane. Of these components, sterols play a significant role in modifying the mechanical properties of membranes. Sterols, in particular cholesterol, were known for a long time to generally increase the $k_{c}$ of the membrane. They were however later found to have different effects depending on the type of the host lipid. For instance, it has been shown that the rigidifying effect of cholesterol increases with the number of saturated lipid chains in the membrane. This effect is less apparent for lipids with one monounsaturated chain and for lipids with two monounsaturated chains cholesterol has essentially no effect on the $k_{c}$. The effect of cholesterol on the bending rigidity of membranes is treated in more detail in Chapter 4. 


\subsection{Statement of the Problem}

Biomechanical properties of cells such as cellular stiffness have been increasingly considered as biomarkers for diseases. For instance, stiffness of cancer cells has been correlated to the malignant potential in certain cell lines. Likewise, various other conditions have been associated with changes in the cellular stiffness, including coronary heart diseases, parasitic infections, red blood cell anemia, and neurodegenerative diseases. Separation of the cells affected with these conditions or the extracellular vesicles involved in mediating these conditions provides valuable clues into the pathology of the disease and is of great diagnostic and therapeutic values.

Acoustophoresis as a simple, gentle, and high-throughput separation tool is well positioned to help in the separation of cells and other bioparticles. This method however, despite its intrinsic capability in making differentiation based on compressibility, has been shown to be ineffective in separation of bioparticles which differ to one another in mechanical properties. Therefore, unaltered cell separation using acoustophoresis has been restricted mainly to size contrast. The main observation emerging from studies involving cell separation was that different cell types seemed to behave similarly, in that in all the studies the cells would tend to migrate toward the high-pressure zone(s) or node(s) of the ultrasonic standing wave. Cellular rigidity, however, varies drastically among cells, changing as much as 10 folds in stiffness in some instances. To our knowledge, no previous works were able to take advantage of this vast change for same size and shape cells to achieve separation in acoustophoresis.

The initiative undertaken in this study will be a departure from conventional acoustofluidic technologies in that for the first time thermally-induced changes in the physical properties of membrane-bound bioparticles will be harnessed to yield separations that are otherwise impossible using conventional methods. Here, to differentiate between vesicles of different sources and compositions, we will rely on the sensitive response of vesicles' compressibility due to temperature changes. Due to their phase change behaviors, vesicles are expected to undergo transitions from 
positive to negative $\phi$ at a unique temperature based on their composition. This transition will be caused by ordered to disordered phase transition in response to heating, causing the membrane of the vesicles to become more compressible or less stiff. Therefore, when a mixture of two distinct vesicle compositions with distinct $T_{\text {transition }}$ properties are injected into the microfluidic device, if the device is operated at a separation temperature where $T_{\text {separation }}$ is in between the $T_{\text {transition }}$ of the vesicles then separation can be achieved. Figure 1.24 depicts the expected separation of two types of vesicles, each experiencing a different $\phi$ sign at $T_{\text {transition,ves1 }}<T_{\text {separation }}<$ $T_{\text {transition,ves2 }}$. It is anticipated that as long as a safe temperature range is employed $\left(1-43^{\circ} \mathrm{C}\right)$, no permanent change to the genetic or proteomic properties of the biological vesicles will occur.

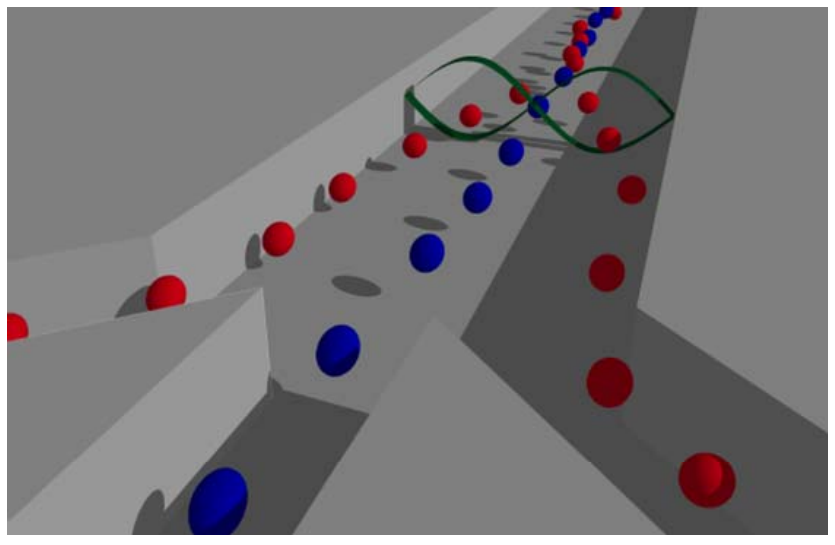

Figure 1.24 Separation in this study is expected to be realized as each of the two types of vesicles experiences a different $\phi$ at $T_{\text {transition,ves1 }}<T_{\text {separation }}<T_{\text {transition,ves2 }}$. 


\section{Materials and Methods}

\subsection{Materials}

\subsubsection{Microfluidic Device Substrate}

The choice of material is of great importance in ensuring a reliable acoustic resonance in the microfluidic channel. The devices used in this study featured a transversal design, in which the standing waves are established mainly by the reflections from the walls of the channel. This mode of resonance, i.e. perpendicular to the actuation direction, relies on materials with high characteristic acoustic impedance such as silicon, metals or glass, but since the resonance is achieved by the substrate as the main resonating body, the relative thickness of the layers is not crucial. What makes a transversal resonator successful, however, is the proper choice of materials that exhibit low acoustic losses and a high difference in acoustic impedance compared to the fluid. ${ }^{92}$ Figure 2.1 shows a schematic illustration of a transversal resonator. For a half-wavelength resonance, the optimum width for the bulk material surrounding the channel is $3 / 4$ of the wavelength.

The substrates used for the microchannel fabrication were 4 -inch $<100>$ silicon wafer purchased from WRS Materials. The wafers were 500-550 $\mu \mathrm{m}$ thick and pre-coated on both sides with a low-stress silicon nitride $\left(\mathrm{Si}_{3} \mathrm{~N}_{4}\right)$ layer. The silicon nitride layer, which was $1.000 \pm 0,050$ $\mu \mathrm{m}$ thick, served only as a mask during the potassium hydroxide $(\mathrm{KOH})$ etching of the underlying silicon and was ultimately stripped off from the silicon through another wet etching process.

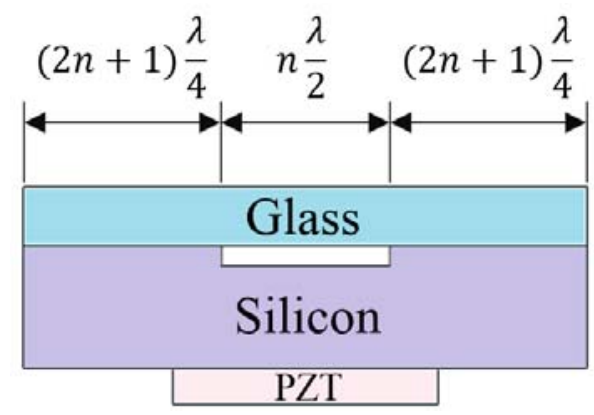

Figure 2.1 Schematic of a transversal resonator, showing the optimum dimensions of the resonating substrate. 


\subsubsection{Device Cover Lid}

To minimize the acoustic losses caused by the reflection from surfaces, the acoustic impedance of two adjacent layers in a resonator should be matched such that the maximum energy density of the acoustic radiation is achieved in the fluidic layer. ${ }^{92}$ One of the most common materials used as the cover lid in acoustic resonators is borosilicate glass, which not only provides good visualization but also due to its strong bonding with the silicon achieved by anodic bonding, make the entire structure resonating as one single body. In this study, 4-inch BOROFLOAT ${ }^{\circledR} 33$ borosilicate glass double-side polished wafers (Präzisions Glas \& Optik GmbH) were used as the cover lid of the silicon wafers. The thickness of the wafers was $0.700 \pm 0.025 \mathrm{~mm}$. The BOROFLOAT $^{\circledR}$ wafer, which is made of borosilicate glass, has an almost identical coefficient of thermal expansion as silicon, has very close acoustic impedance to that of silicon, and is very resistant to high temperature, making it a good candidate for anodic bonding with silicon.

\subsubsection{Piezoelectric Transducer}

Lead zirconate titanate (PZT), which is an intermetallic inorganic compound with marked piezoelectric properties, was used as the acoustic transducer in this study. Table $2-1$ presents the specifications of the transducers which were purchased from APC International. Both APC 850 and 851 material types are PZT-based compositions, $\left(\mathrm{Pb}\left[\mathrm{Zr}_{x} \mathrm{Ti}_{1-x}\right] \mathrm{O}_{3}, 0 \leq x \leq 1\right)$ with perovskite structure, whose properties have been optimized for specific applications through adjusting the zirconia:titania ration, and/or by adding proprietary secondary phases. Disk-shaped transducers featured a wrap-around electrode pattern, allowing the attachment of electrical leads to one side of the transducer, whereas the plate transducer was of solid pattern.

Table 2-1 Specifications of the piezoelectric transducers used in this study.

$\begin{array}{cccccc}\text { Shape } & \text { Diameter/ Length } & \text { Width } & \text { Thickness } & \text { Electrode Pattern } & \text { Material } \\ \text { Disk } & 12.75 \mathrm{~mm} & \text { N/A } & 2.00 \mathrm{~mm} & \text { Wrap Around } & 850 \\ \text { Disk } & 9.50 \mathrm{~mm} & \text { N/A } & 2.20 \mathrm{~mm} & \text { Wrap Around } & 850 \\ \text { Plate } & 70.00 \mathrm{~mm} & 13.00 \mathrm{~mm} & 0.70 \mathrm{~mm} & \text { Solid } & 851\end{array}$




\subsubsection{Lipids Used for Vesicles Preparation}

For the preparation of vesicles, three phosphatidylcholines (PCs) containing linear saturated fatty acyl chains namely, 1,2-dilauroyl-sn-glycero-3-phosphocholine (DLPC), 1,2dimyristoyl-sn-glycero-3-phosphocholine (DMPC) and 1,2-dipalmitoyl-sn-glycero-3phosphocholine (DPPC), one phosphatidylcholine containing one monounsaturated and one linear saturated fatty acyl chains namely 1-palmitoyl-2-oleoyl-sn-glycero-3-phosphocholine (POPC), and one phosphatidylcholine containing two monounsaturated fatty acyl chains, namely 1,2-dioleoylsn-glycero-3-phosphocholine (DOPC) were purchased from Avanti Polar Lipids (Alabaster, AL). All the lipids bought from Avanti had purities over $99 \%$ and were used without further purification. Lecithin was purchased form Sigma-Aldrich (L- $\alpha$-phosphatidylcholine from egg yolk, $\geq 99 \%$ lyophilized powder) and was used for the preliminary testing of the device. Cholesterol (powder, BioReagent, $\geq 99 \%$ ), used as the stiffening agent, was purchased from Sigma-Aldrich. Table AI1 in Appendix I presents the name, synonyms, molecular formula and weight of the lipids used in this study.

Table 2-2 shows the molar percent of three of the PCs used in this study in a number of naturally occurring membranes. The main PCs used in this study, i.e. DMPC, DPPC, and POPC, are among the most studied PCs in the literature with reports on their mechanical and thermotropic behaviors that were essential for comparisons and calculations in this work. While all of these PCs are common in naturally-occurring membranes, DPPC is one of the most abundant as shown in Table 2-2. Other than DPPC, 1,2-distearoyl-sn-glycero-3-phosphocholine (DSPC), 1,2-dioleoylsn-glycero-3-phosphocholine (DOPC), and 1,2-dilinoleoyl-sn-glycero-3-phosphocholine (DLPC) are some of the other abundant PCs usually found in naturally-occurring membranes ${ }^{69}$ which, except for DSPC, were selected in this study for the evaluation of their thermo-acoustofluidic behavior, but they were not among the main PCs studied for the lack of mechanical and thermotropic data. 
Table 2-2 Natural occurrence of DMPC, DPPC, and POPC (molar\% of the total PCs in the corresponding membrane).

$\begin{array}{ccccc}\text { Tissue } & \text { DMPC } & \text { DPPC } & \text { POPC } & \text { Ref } \\ \text { Human, erythrocyte membrane } & 0.4 & 33.3 & \text { N/A } & 93 \\ \text { Human, platelet membrane } & \text { N/A } & 31.4 & \text { N/A } & 94 \\ \text { Rat, liver cells membrane } & 0.5 & 30.2 & \text { N/A } & 95 \\ \text { Saccharomyces cerevisiae, BY4741 } & \text { N/A } & 0.09 & 2.92 & 96 \\ \text { Canine, kidney epithelial cells membrane } & \text { N/A } & \text { N/A } & 0.38 & 97\end{array}$

\subsubsection{Labeling Dyes}

Fluorescent labeling was used in this study to better visualize the vesicles in the microfluidic channel. Since the host medium of the vesicles is an aqueous suspension, all the dyes used were lipophilic such that they selectively tag only the lipid membrane of the vesicles. Nile red (9-diethylamino-5-benzo[ $\alpha]$ phenoxazinone), also known as Nile blue oxazone, was one of the dyes used, which was bought from Acros Organics and was applied to the vesicles during the preparation steps. Nile red is a strongly red-fluorescent dye, which does not interact with the lipids it is supposed to reveal. ${ }^{98}$ This dye, which is mainly used to label the intracellular lipids and hydrophobic domains of proteins, has excitation and emission peaks of 553 and $636 \mathrm{~nm}$, respectively. Figure 2.2 shows the molecular structure and fluorescence emission of Nile red in tetrahydrofuran (THF). ${ }^{99}$
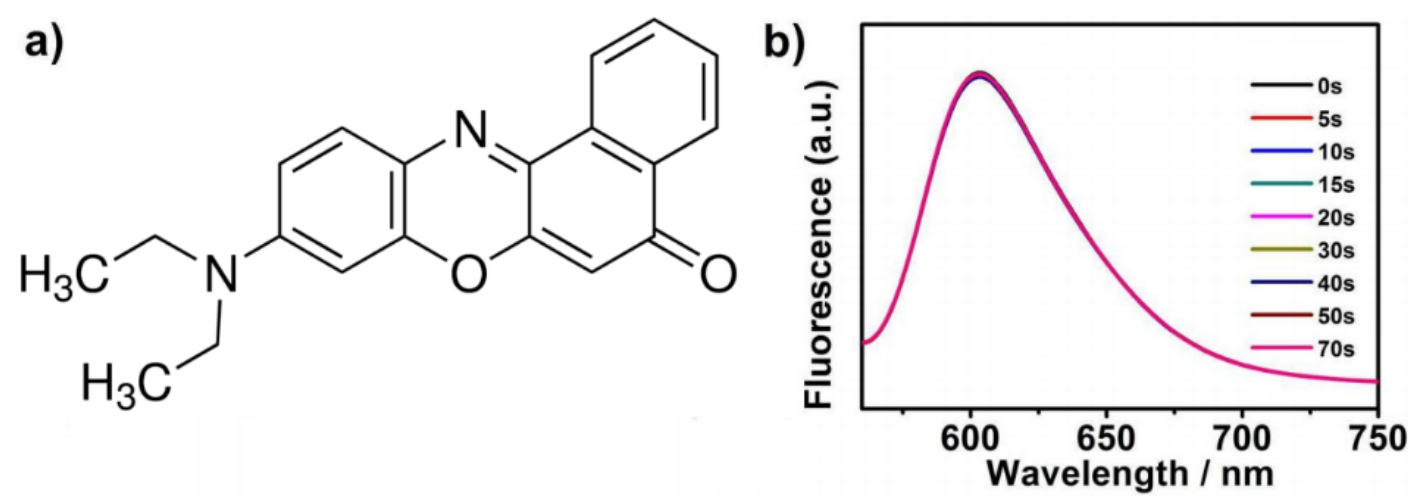

Figure 2.2 a) Molecular structure of Nile red. b) Fluorescence emission spectra of Nile Red in THF under visible light excitation $\left(450 \mathrm{~nm}, 7 \mathrm{~mW} \mathrm{~cm}^{-2}\right)$. (Reprinted with permission from [99]) 
The other dyes used in this study were blue-fluorescent Laurdan (6-dodecanoyl-2dimethylaminonaphthalene) and green-fluorescent DiOC6(3) iodide [3,3-dihexyloxacarbocyanine iodide], which were bought from AnaSpec, Inc. Analogous to Nile red, these two dyes are mainly used for the labeling of cellular membranes and lipids. Figure 2.3 shows the molecular structure of Laurdan and DiOC6(3) iodide. Given the small difference between the electronegativity of $\mathrm{C}$ and $\mathrm{H}$, and the zero difference between $\mathrm{C}$ and $\mathrm{C}$, no charge separation exists on the benzene ring, leading to no polarity on the ring. Therefore, as can be seen in Figure 2.2 and Figure 2.3, the large ring structure on these dyes render them hydrophobic and therefore they partition inside the membrane. Fluorescence intensity of Laurdan is sensitive to the physical state of the phospholipid membrane, making it emit at different wavelengths when incorporated into the membrane in gel and fluid states. ${ }^{100}$ In other words, the steady-state excitation and emission spectra of Laurdan can be split into two separate components corresponding to the gel and the liquid crystalline phases. ${ }^{101}$ The difference in emission spectra is specially of great interest as it can be used to monitor the interconversion between the two phases. ${ }^{101}$ In methanol, Laurdan has excitation and emission peaks of 366 and $497 \mathrm{~nm}$. DiOC6(3) iodide, which is often used to stain mitochondria and the endoplasmic reticulum (ER) in mammalian cells, ${ }^{102}$ has excitation and emission peaks of 482 and $504 \mathrm{~nm}$ in methanol.
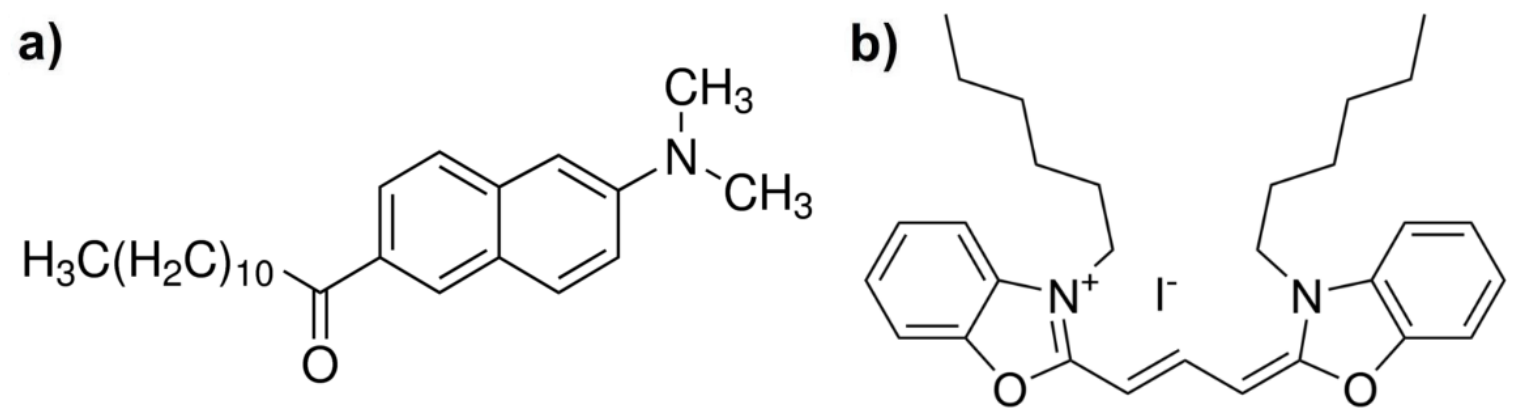

Figure 2.3 Molecular structure of a) Laurdan, which is known to incorporate into the hydrophilichydrophobic interface of the membrane having its 12-carbon long tail, known as lauric acid, anchored in the acyl chains region. b) DiOC6(3) iodide molecule. 


\subsection{Microfabrication}

As mentioned earlier in Section 2.1, the thermo-acoustofluidic devices used in this study consisted of a microfluidic channel etched into a silicon substrate and then covered with a glass cover lid. The microfluidic channels were fabricated using a standard photolithography recipe, which is detailed in this section.

\subsubsection{Pattern Generation}

The main device used in the thermo-acoustofluidic studies, was a single-stage microfluidic device with one main channel splitting into three narrower channels, featuring a three-pronged configuration. The center channel in the three-pronged structure, which is the extension of the main channel runs through the first outlet, and the two side channels diverged from the split at a $45^{\circ}$ with respect to the main channel's axis meet at the second outlet, making the channel capable of performing two-type separations. Figure 2.4 shows the layout of the 1-stage separation device drafted by the Layout Editor software (Juspertor $\mathrm{GmbH}$ ). For the two-stage separations, another microfluidic channel featuring a similar architecture but with an extra three-pronged split was used in the experiments, which enabled two consecutive two-type separations.

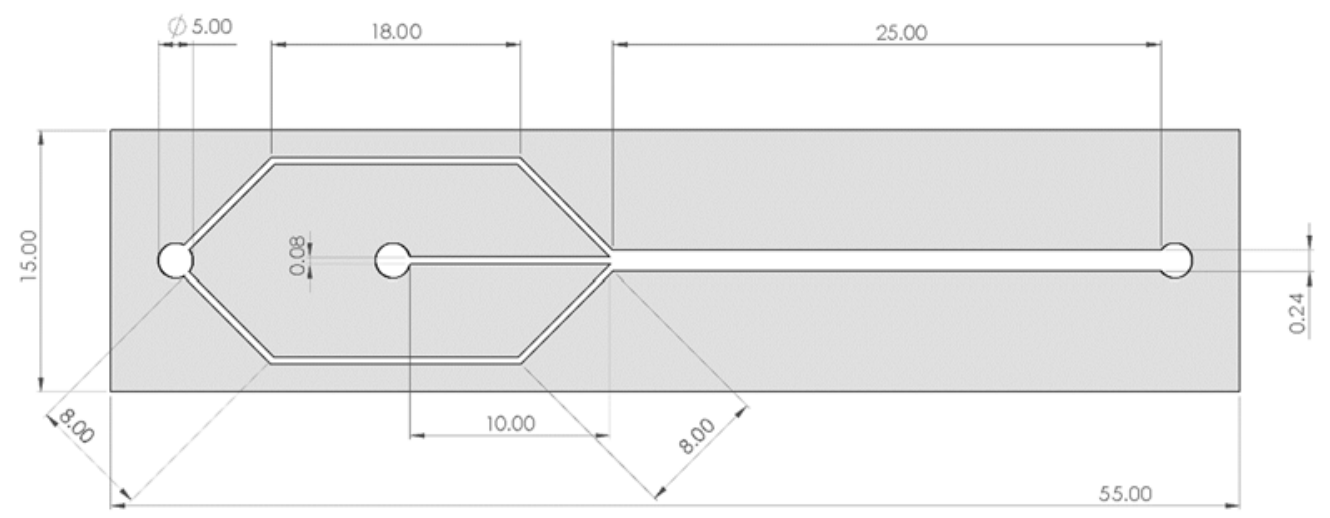

Figure 2.4 The layout of the 1-stage three-pronged microfluidic device, showing the inlet on the right side and the two outlets on the left. The dimensions are the mask-design dimensions in $\mathbf{m m}$. The drawing is not to scale to better show the details. 
The design dimensions of the channels were calculated such that after the $\mathrm{KOH}$ etching the final width of the main channel was between 450 to $500 \mu \mathrm{m}$ and the average width of the side channels was around $180 \mu \mathrm{m}$. The length of the main channel extended from the inlet to the split was around $25 \mathrm{~mm}$.

To provide the main channel of the microfluidic channel with completely vertical side walls, the drafted layout was rotated by $45^{\circ}$ such that the walls of the etched channel were $\{100\}$ planes. The two side channels slanted at $45^{\circ}$ with respect to the main channel's axis were however of trapezoid cross-section with $\{111\}$ side walls. Figure 2.5 shows how the reorientation of the microfluidic channels on the $<100>$ silicon wafer results in vertical planes for the main separation channel, which is a prerequisite for the establishment of a powerful standing wave.

In the next step, a photomask (soda lime glass) pre-coated with a $1000 \mu \mathrm{m}$ thick chromium layer and $5300 \AA$ thick positive-tone photoresist (AZ1518) was patterned using a mask maker ( $\mu$ PG 101 Heidelberg Instruments Mikrotechnik GmbH). Patterning was usually performed using a 100\% exposure rate with $12 \mathrm{~mW}$ of power. After transferring the pattern to the mask, the photoresist on the mask was developed in a developer ( $\mathrm{AZ}^{\circledR} 400 \mathrm{~K}$, $\mathrm{AZ}$ Electronic Materials) diluted with water (4:1) for 70 seconds. An etchant (Chromium Etchant 1020, Transene Company, Inc.) was then used to remove the chromium layer through the area without photoresist. For doing so, fully concentrated etchant was poured inside a vial and wafer was then washed with the etchant for 1 minute. The remaining photoresist was in the next step stripped via oxygen plasma treatment, using 400 mTorr pressure of $\mathrm{O}_{2}$ and 100 watts of power with $60 \mathrm{sccm}$ flow rate applied for 660 seconds. Alternatively, the remaining photoresist could be stripped from the mask using a Remover PG solution (MicroChem) kept at $60{ }^{\circ} \mathrm{C}$ for 30 minutes.

Before processing the wafers, a spin cleaning was performed on the wafers using acetone, isopropyl alcohol (IPA) and bis(trimethylsilyl)amine (also known as hexamethyldisilazane, or HMDS) at $3000 \mathrm{rpm}$ for 30 seconds. HMDS was used as an adhesion promoter for the photoresist. 


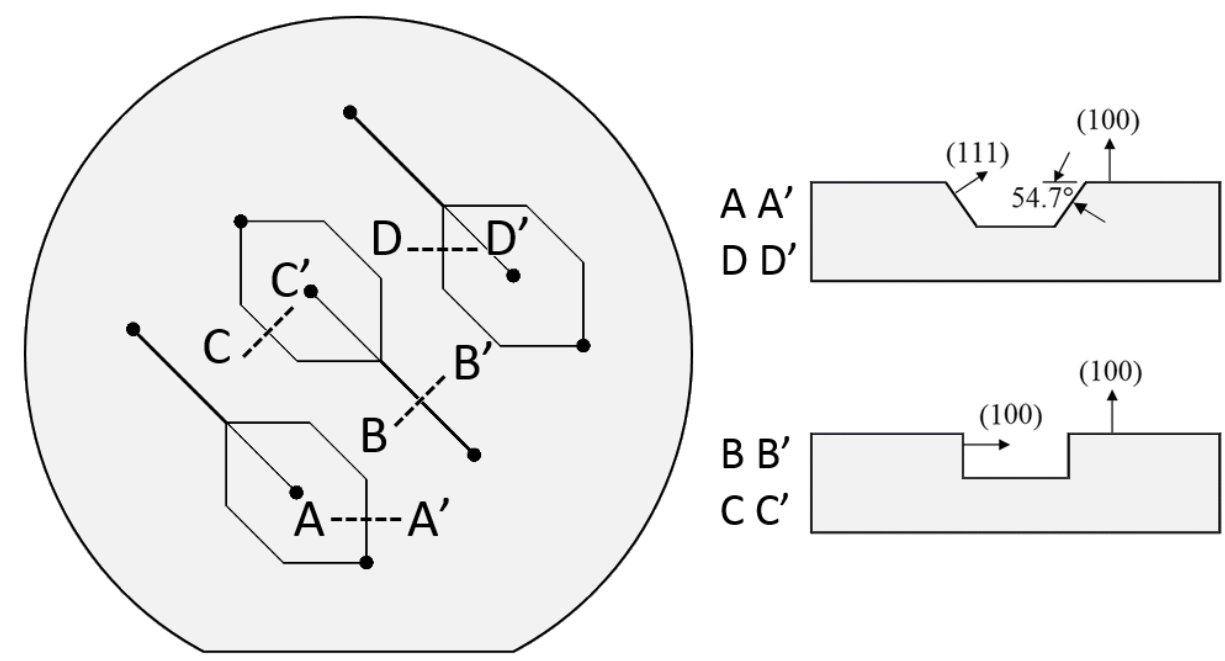

Figure 2.5 Orientation of the microfluidic channels' layouts on the (100) silicon wafer, yielding a trapezoid cross section for AA' and DD' and a rectangular cross section for BB' and CC' after KOH etching.

After cleaning the wafers and approving the feature quality on the mask by optical microscopy, the front side of the silicon wafers was first spin coated by photoresist AZ 4620 (MicroChemicals GmbH). The photoresist was applied to the surface in two consecutive steps: 1) 5 seconds at $100 \mathrm{rpm} / \mathrm{s}$ up to $500 \mathrm{rpm}$ and 2) 30 seconds at $300 \mathrm{rpm} / \mathrm{s}$ up to $2500 \mathrm{rpm}$, followed by a soft bake at $110{ }^{\circ} \mathrm{C}$ for $2 \mathrm{~min}$. A contact-mode mask aligner (Model $800 \mathrm{MBA}, \mathrm{OAI}$ ) was employed to expose the front side with the recommended exposure energy of $400 \mathrm{~mJ} / \mathrm{cm}^{2}$. Wafers were then developed in the proper developing solution ( $A Z^{\circledR} 400 \mathrm{~K}$, AZ Electronic Materials) prediluted with DI water at a volume ratio of 1:4. The developed wafers were subsequently assessed via optical microscopy (Unitron Versamet Optical Microscope), followed by a descum plasma treatment using oxygen as the process gas to ensure the complete removal of the resist from the channels' trench. The process was done using a reactive ion etcher (CS-1701, MARCH) with 400 watts of power and $100 \mathrm{~m}$ Torr pressure of $\mathrm{O}_{2}$ entering the chamber with $60 \mathrm{sccm}$ of flow rate for 1 minute. The cleaned channels were then processed in the same instrument this time with tetrafluoromethane $\left(\mathrm{CF}_{4}\right)$ plasma to remove the nitride layer. After removing the nitride layer, the 
remaining photoresist was stripped from the wafers using a Remover PG solution (MicroChem) kept at $60{ }^{\circ} \mathrm{C}$ for 10 minutes. The wafers were then rinsed thoroughly with IPA followed by water rinse and dry.

\subsubsection{Etching}

After approving the features quality via optical microscopy, the underlying silicon material on the wafers was removed by an anisotropic wet etching using a preferential silicon etchant (PSE200) (Transene Company, Inc.). The wet etching was stopped when the desired depth (90 $\mu \mathrm{m})$ was achieved. The depth of the channel was measured by a profilometer (Alpha-Step 200, KLATencor). The wafers were then rinsed with ethanol and DI water. After drying the wafers, the remaining nitride layer on the wafers was removed by another wet etching process using an aluminum etchant (Transene Company, Inc.) kept at $180{ }^{\circ} \mathrm{C}$ for 210 minutes. After removing the nitride layer, wafers were again rinsed with a piranha solution (3 parts of sulfuric acid $\left(\mathrm{H}_{2} \mathrm{SO}_{4}\right)$ and 1 part of $30 \%$ hydrogen peroxide $\left.\left(\mathrm{H}_{2} \mathrm{O}_{2}\right)\right)$, ethanol and DI water to remove any debris left on the wafers. Following that, optical microscopy was again used to examine the wafers. Figure 2.6 shows the photomask after the chromium layer and photoresist removal and also a patterned wafer before and after etching in $\mathrm{KOH}$. Scanning electron micrographs of the etched silicon wafers are also shown in Appendix II.

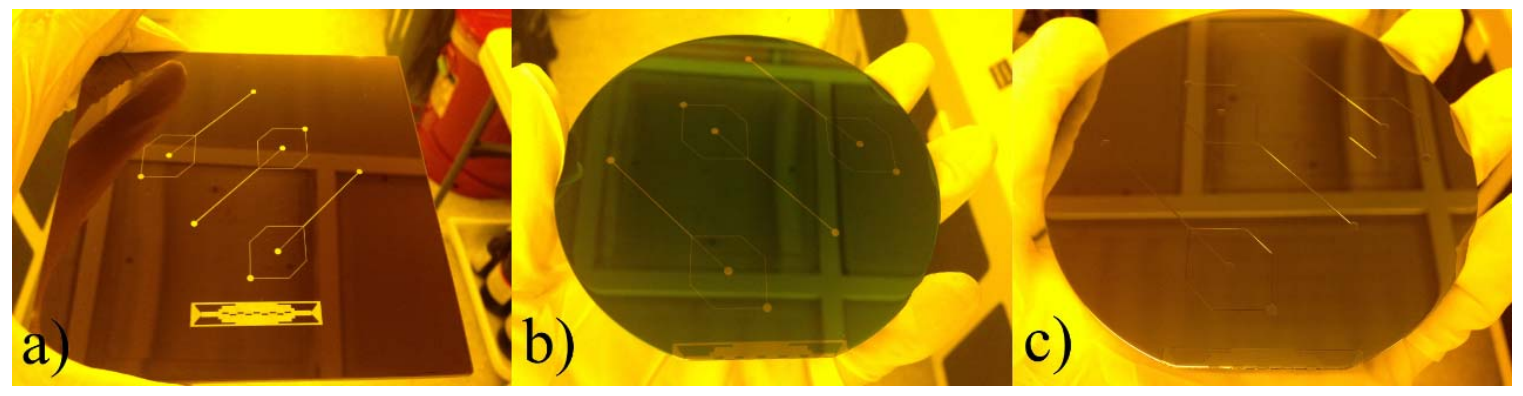

Figure 2.6 Different steps of the channel microfabrication: a) The photomask used for the pattern generation after the photoresist removal. b) A patterned wafer before etching. Green color is for the nitride layer. c) The same wafer, after silicon etch and nitride removal. 


\subsubsection{Bonding of Glass Cover Lid}

In the last step, the inlets and outlets were manually bored inside the BOROFLOAT ${ }^{\circledR}$ glass cover lids using an ordinary drill (220-01 WorkStation ${ }^{\mathrm{TM}}$, Dremel). Both the silicon wafers and cover lids were then carefully cleaned in the piranha solution (3 parts of sulfuric acid $\left(\mathrm{H}_{2} \mathrm{SO}_{4}\right)$ and 1 part of $30 \%$ hydrogen peroxide $\left.\left(\mathrm{H}_{2} \mathrm{O}_{2}\right)\right)$, followed by a rinse with acetone and DI water. After drying the wafers, the glass wafers were then anodically bonded to the heated-up substrates (500 ${ }^{\circ} \mathrm{C}$ ) using $500 \mathrm{~V}$ of potential difference provided by a power supply (Model 247, Keithley Instruments Inc.) for 15 minutes. After bonding, the bonded wafers were slowly cooled down to room temperature in air. The main drawback of anodic bonding is the limited allowable difference in the coefficient of thermal expansion (CTE) of the bonded materials. Therefore, as mentioned earlier, the CTE of the processed glass needs to closely match that of the bonding wafer. The CTE of the glass wafers used in this study (BOROFLOAT ${ }^{\circledR} 33$ borosilicate glass) was $3.25 \times 10^{-6} \mathrm{~K}^{-1}$ in the temperature range $20-300^{\circ} \mathrm{C}$, which is very close to that of silicon $\left(2.56 \times 10^{-6} \mathrm{~K}^{-1}\right)$ in the same temperature range. Figure 2.7 presents the process flow of the microfluidic channel fabrication, summering the important steps of the microfabrication.

\subsection{Thermo-Acoustophoretic Setup}

A solder wire (Sn-3.0Ag-0.5Cu) was used to attach the electrical leads (AWG TFE 3") to the piezoelectric transducer employing a soldering station (968A+, AOYUE) set at $400{ }^{\circ} \mathrm{C}$. After attaching the leads, the piezoelectric transducer was glued from the sides by a cyanoacrylate glue (495 ${ }^{\text {TM }}$ Super Bonder ${ }^{\circledR}$, Loctite) to the back side of the chip underneath the main separation channel. A function generator (DG4062, RIGOL Technologies Inc.) capable of generating sinusoidal AC signals was connected to a power amplifier (Model 2348, TEGAM Inc.) to amplify the generated signal. The piezoelectric transducer was then connected to the power amplifier. The applied voltage amplitudes were simultaneously monitored using an oscilloscope (TDS 2014B, Tektronix Inc.). 
- Wafer preparation

- Pattern transfer

- Photoresist development

- Nitride removal

- Silicon etch

- Photoresist and nitride strip

- Anodic bonding

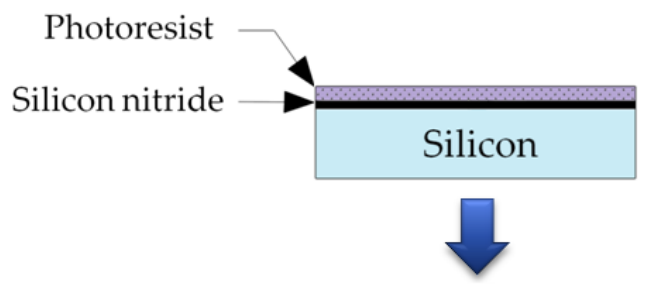

Film mask

UV
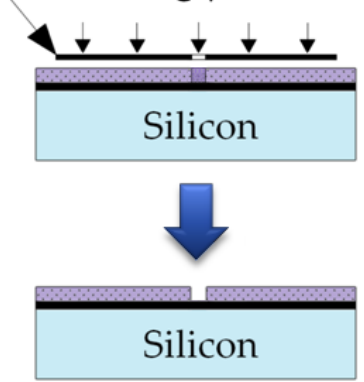

Silicon

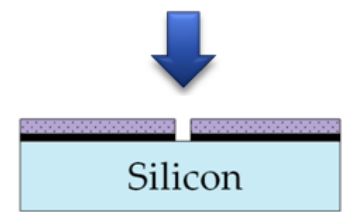

Silicon
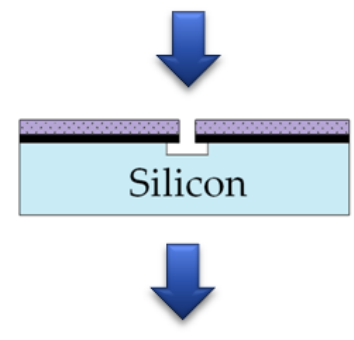

Silicon

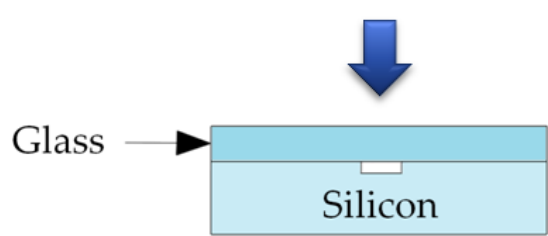

Figure 2.7 Schematic of the process flow of the fabrication of the microfluidic channels used as the separation device. The front side of the silicon wafer pre-coated with silicon nitride layer was first cleaned with acetone, IPA and HMDS and then was spin coated by a photoresist, followed by a softbake at the recommended temperature. The photoresist was subsequently exposed and developed in the developer solution. The developed pattern was etched with carbon tetrafluoride plasma to remove the revealed nitride layer. The underlying silicon material was subsequently removed via wet-etch processing in $\mathrm{KOH}$. To remove the remaining nitride layer on the surface, the wafer was processed with an aluminum etchant, followed by a cleaning in piranha solution. After rinsing and drying, the wafer was examined via optical microscopy to approve the feature quality. Finally, the wafer was cleaned carefully and anodically bonded with a borosilicate glass wafer on top. 


\subsubsection{Temperature Management of the Device}

Chip temperature was controlled throughout all experiments using a Peltier element (Farnell) connected to a DC power supply (Model 72-2010, TENMA). The temperature stability of the microchannel was improved using two heat sinks, above and below the Peltier element. At the frequency range around 1.4 MHz, which was the common working frequency in this work, the heat generated due to the ultrasonic actuation lead to 3 to $4{ }^{\circ} \mathrm{C}$ increase in the temperature and the Peltier element was adjusted accordingly.

The temperature of the system was measured using a flat platinum resistance thermometer (PT100, Thermometrics) with the repeatability of $\pm 0.1^{\circ} \mathrm{C}$, fixed to the back side of the chip near the main channel. The registered temperature was confirmed by an infrared thermometer on top of the channel. Figure 2.8a shows a schematic exploded view of the device showing the transducers and the heat sink.

\subsubsection{Liquid Handling}

For separation runs, the inlet and outlet flows were controlled by syringes (BD LuerLok ${ }^{\mathrm{TM}}$ ) connected to two syringe pumps (New Era Pump Systems Inc.). One pump was connected to the chip inlet, infusing the vesicle specimen from a 3-ml syringe. The other pump was set in withdrawal mode and connected to two plastic syringes to control the flow in the separation chip. Both pumps were connected via PVC tubing with luer ends (TUBING LUER M-F 24", ColeParmer, Vernon Hills, IL) to the chip outlets.

\subsubsection{Visualization of the Separation Experiments}

A fluorescence microscope (Axio Scope.A1, Carl Zeiss Microscopy GmbH) fitted with three filters, namely 4',6-diamidino-2-phenylindole (DAPI), fluorescein isothiocyanate (FITC) and rhodamine, was employed to visualize the samples during the thermo-acoustofluidic experiments. Separations were captured and recorded at 28 frames per second by a digital camera (AxioCam ICc 
1, Carl Zeiss Microscopy GmbH) mounted on the microscope. The same microscope, operating in transmission and reflected modes, was also used to examine the formation and size distribution of the newly-prepared giant vesicles. Figure $2.8 \mathrm{~b}$ shows the 1 -stage thermo-acoustofluidic device loaded onto the stage of the fluorescent microscope, along with the luer fittings and the fluidic tubing.
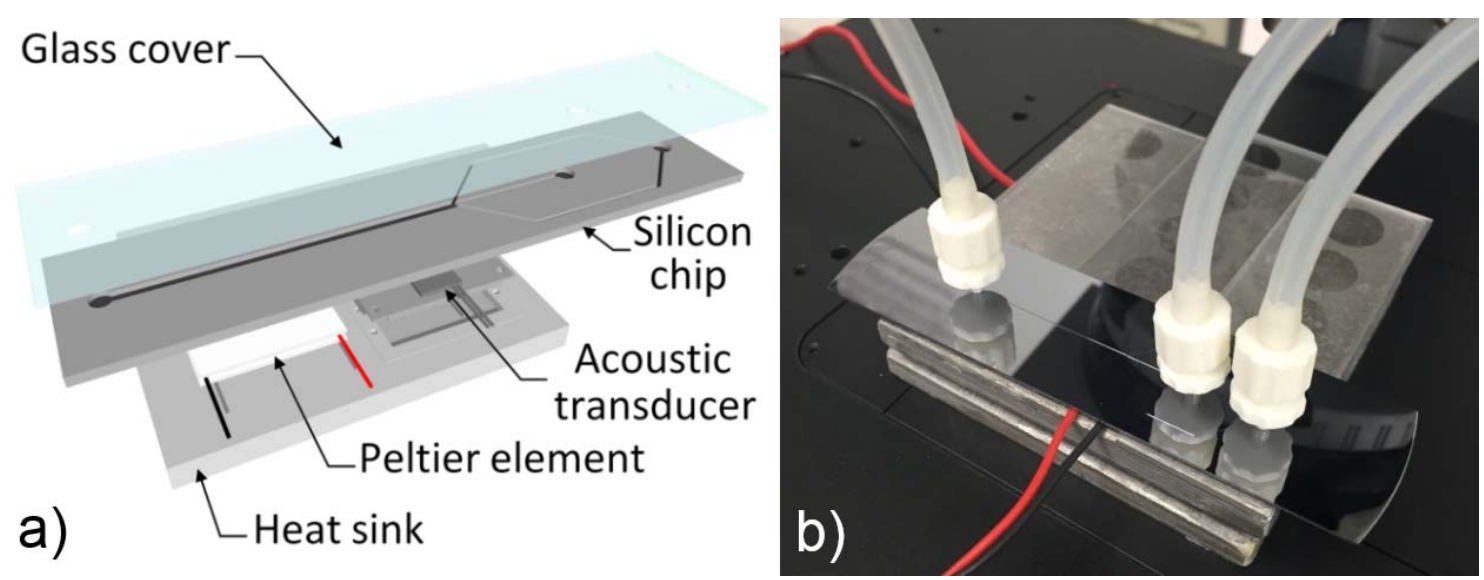

Figure 2.8 a) Schematic exploded view of the thermo-acoustofluidic device used for the 1-stage separation studies, featuring a thermoelectric transducer, piezoelectric transducer, and the heat sink. b) Actual image of the device, showing the chip along with the luer fittings and the fluidic tubing.

\subsection{Preparation of Vesicles}

A modified solvent-injection method, inspired mainly by the so-called ethanol-injection method, ${ }^{103,104}$ was employed in this study to prepare the vesicles. Using this method, lipid vesicles were obtained starting with a solution of the desired lipid(s), in the form of a lyophilized powder and combined in the appropriate ratio, prepared in the water-miscible solvent tetrahydrofuran (THF) ( $\geq 99.9 \%$, inhibitor-free, Sigma-Aldrich). The weight percentage of the total lipids in tetrahydrofuran was between 1 to $3 \%$. In the next step, deionized water was added dropwise to the solution under mixing using a magnetic stirrer. Controlled by a syringe pump (New Era Pump Systems, Inc.), the dripping velocity of water addition was set to $10.0 \mathrm{ml} / \mathrm{h}$. At approximately 25 35 volume $\%$ THF, the addition of water was stopped and the remaining solvent was allowed to 
evaporate in ambient conditions overnight. Fluorescent tagging of vesicles was achieved by the addition of the desired dye to the starting lipid/THF solution. Cholesterol incorporation for the samples containing cholesterol was done the same way. Optical microscopy, operating in transmission and reflected modes, was employed to confirm the formation and size of the prepared vesicles (Figure 2.9).

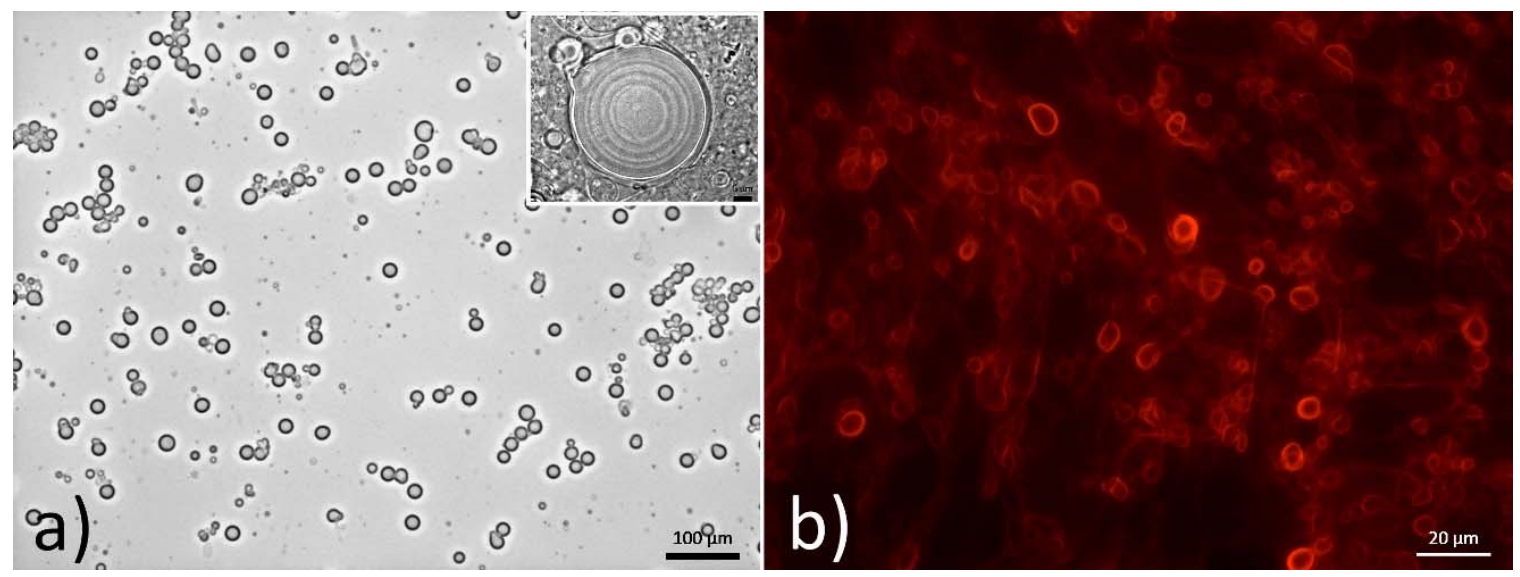

Figure 2.9 Optical micrographs of the DMPC vesicles: (a) Transmission-mode image showing a uniform population of vesicles (The inset shows a giant multilamellar vesicle in higher magnification) and (b) fluorescent image of the same sample.

The method described here yields a homogenous population of unilamellar and multilamellar vesicles and was shown to be capable of yielding a wide range of sizes depending on the lipid concentration, water content, and other conditions. Figure 2.10 shows a fluorescent $2.5 \mathrm{D}$ micrograph of the giant DPPC vesicles prepared with the described methods, revealing the existence of both unilamellar and multilamellar vesicles in the prepared sample. The exact mechanism by which giant vesicles form by solvent-injection method is not known. It has been reported, however, that self-assembly causes the formation of small vesicles at low water content, and then as those vesicle fuse together giant vesicles form. ${ }^{105}$ 


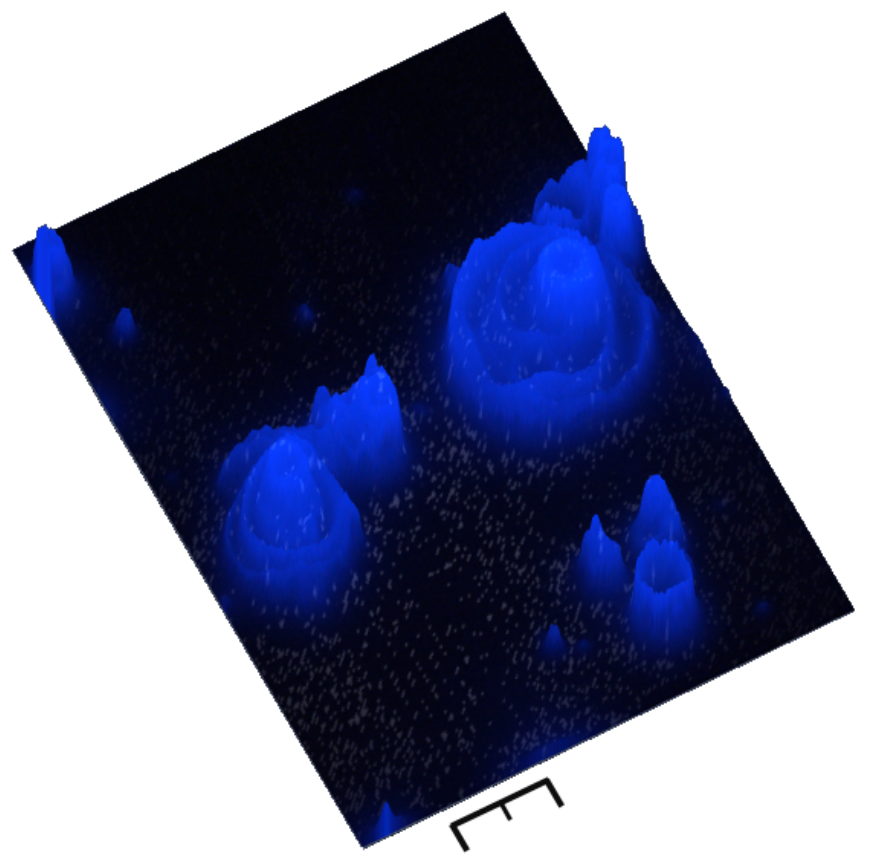

Figure 2.10 2.5D view micrographs of the relative fluorescence intensity of the DPPC GVs revealing the lamellarity of the vesicles. The prepared vesicle batch contained both unilamellar and multilamellar GVs (Scale bar is $10 \mu \mathrm{m})$. 


\section{Thermally-Assisted Acoustophoresis of Phosphatidylcholine Vesicles}

\subsection{Introduction}

The lipid bilayer membrane is the common underlying structure that confines the cytoplasm and cellular organelles and gives cells their unique physical properties such as size, density, and stiffness. ${ }^{64}$ Various biological changes in cells can affect these properties such as aging, ${ }^{106}$ response to an infection, ${ }^{107}$ or drug treatment. ${ }^{108}$ Changes in stiffness are especially of importance due to recent discoveries linking these changes to invasiveness of cancers, ${ }^{109}$ parasitic infections, ${ }^{110}$ and red blood cell anemia. ${ }^{111}$ The ability to separate cells which have undergone these changes in a viable and unaltered state from their healthy counterparts can be of significant importance to many biomedical fields. Since vesicles are essentially lipid bilayer membranes encapsulating aqueous cores, they have been chosen to be the subjects of this study in which their properties have been altered to test the feasibility and limitations of thermally-assisted ultrasonic separation. Standing-wave acoustophoresis has been previously reported for various microparticle separations, primarily based on their size and density. ${ }^{43,112,113}$ Its label-free separation and gentle action on the particles provided most of its development impetus, particularly in the field of cell separations. ${ }^{52} \mathrm{~A}$ handful of studies ${ }^{58,114-116}$ have reported practical separation methods; nonetheless, while highlighting numerous limitations and challenges. Unaltered cell separation using acoustophoresis has been restricted mainly to size contrast. ${ }^{51,60}$ The main observation emerging from these studies was that cells often do not vary much in density to yield a change in $\phi$ enough to use in a separation. Altered cells however, by binding them with elastomeric particles yielded a negative $\phi .{ }^{62}$ Similarly by changing the density of the medium by adding large amounts of CsCl salt $\left(220 \mathrm{~g} \cdot \mathrm{l}^{-1}\right)$, red blood cells were separated from platelets. ${ }^{43}$

Cellular rigidity, as an intrinsic property varies drastically among cells, changing as many as 10 folds in stiffness in some instances. ${ }^{117}$ To our knowledge, no previous works were able to 
take advantage of this vast change for same size and shape cells to yield a separation in acoustophoresis. The reason is that at room temperature, cells predominantly have positive acoustic contrast factors, leading to their swift migration towards the nodal region. ${ }^{55}$ For that reason, in this study we introduced temperature as a new dimension to acoustophoresis to yield a change in vesicle compressibility that allowed opposite $\phi$ values for different vesicle systems. Previous works have shown increase in compressibility at higher temperatures in certain cell types e.g. human mesenchymal stem cells, ${ }^{118}$ red blood cells,${ }^{119}$ and human melanoma cells. ${ }^{120}$ We dubbed this approach thermally-assisted ultrasonic separation or thermo-acoustophoresis.

This chapter first discusses the thermally-assisted acoustophoretic behavior of singlecomponent vesicles. Then, it discusses the observation of the same phenomenon for vesicles with binary lipid composition. Lastly, this chapter explores the idea of separating vesicles based on their different thermo-acoustophoretic properties.

\subsection{Single-Component Compositions}

\subsubsection{Thermo-Acoustophoretic Behavior of Lecithin Vesicles}

Pure L- $\alpha$-lecithin or simply lecithin was the first system investigated. The lecithin used was extracted from egg yolk, which typically contains both saturated fatty acids and monoenoic unsaturated fatty acids. The fatty acid composition of the lecithin was 33\% palmitic acid (16:0), $31 \%$ oleic acid (18:1), 15\% linoleic acid (18:2), and 13\% stearic acid (18:0), with other fatty acids having minor contributions, giving rise to an average molecular weight of approximately $768 \mathrm{~g}$.

In a typical experiment, GVs with average diameter of $5 \mu \mathrm{m}$ tagged with DiOC6(3) iodide were prepared from lecithin. The vesicle suspension was then injected into the 1-stage microfluidic channel fabricated following a standard protocol detailed in Section 2.2. The disk-shaped piezoelectric transducer (12.75 $\mathrm{mm}$ in diameter), mounted on the device, was connected to the frequency generator and the chip temperature was lowered down to around $5{ }^{\circ} \mathrm{C}$ with the signal 
generator set to the first harmonic frequency (Figure 3.1a). On actuation of the channel at that frequency and temperature, the GVs were observed to promptly focus at the nodal region located at the center of the channel (Figure 3.1b). A slow sweep of temperature $\left(1^{\circ} \mathrm{C} \min ^{-1}\right)$ was then initiated while the vesicles were visually monitored under the microscope. Once the channel temperature reached or exceeded $29{ }^{\circ} \mathrm{C}$, the GVs collectively migrated towards the anti-nodal regions, located at the walls of the channel (Figure 3.1c). Increasing temperature to more than 50 ${ }^{\circ} \mathrm{C}$ showed no apparent change in the position of the GVs at the walls. On subsequent cooling (-1 ${ }^{\circ} \mathrm{C} \min ^{-1}$ ), however, the GVs were observed to migrate back to the center node of the channel at a slightly lower temperature around $27.5^{\circ} \mathrm{C}$. Since the observed phenomenon was reversible, we hypothesized that due to thermotropic phase transitions in the membrane of the lecithin vesicles, the vesicles had an apparent effect on their mechanical properties, especially compressibility, which manifests itself as a sign change in the $\phi$. The main thermotropic phase transition for the egg yolk lecithin has been however reported to occur at $-15{ }^{\circ} \mathrm{C}$ on the heating curve and at $-7{ }^{\circ} \mathrm{C}$ on the cooling curve. ${ }^{121}$ Given that the egg yolk lecithin is composed of different lipids, it seemed unreliable to correlate the thermotropic acoustic behavior of lecithin to its composition. Therefore, to perform a systematic study with no interference from other properties, GVs were prepared from various single-component PCs with known thermotropic behaviors to conduct a more comprehensive study on the effect of temperature on the acoustic behavior of vesicles.

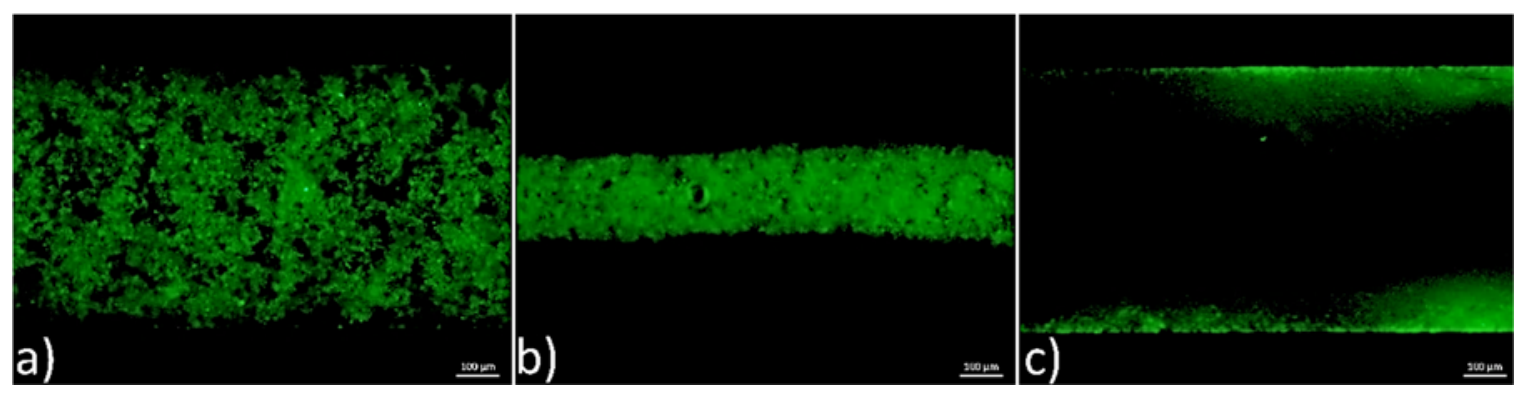

Figure 3.1 Thermo-acoustofluidic behavior of lecithin vesicles: a) at $5^{\circ} \mathrm{C}$ when the acoustic transducer is off. b) at the same temperature with the transducer on. c) at $30.0^{\circ} \mathrm{C}$, vesicles have migrated towards the antinodes. (scale bar is $100 \mu \mathrm{m}$ ) 


\subsubsection{Thermo-Acoustophoretic Behavior of DMPC System}

DMPC or 1,2-dimyristoyl-sn-glycero-3-phosphocholine is a neutral zwitterionic lipid with two saturated fatty acyl chains; illustrated in Figure 3.2 in an ideal and bilayer-inserted conformations constructed using QuteMol ${ }^{122}$ software. DMPC is one of the most well-studied phospholipids having its thermotropic phase transitions at experimentally convenient temperatures (below $25^{\circ} \mathrm{C}$ ). DMPC was chosen as the first lipid among the single-lipid phospholipids to be examined in the thermo-acoustofluidic device. ${ }^{123}$ Giant vesicles with membranes tagged with Nile red were prepared from DMPC. The samples prepared contained unilamellar and multilamellar giant vesicles with the average size of around $10 \mu \mathrm{m}$.
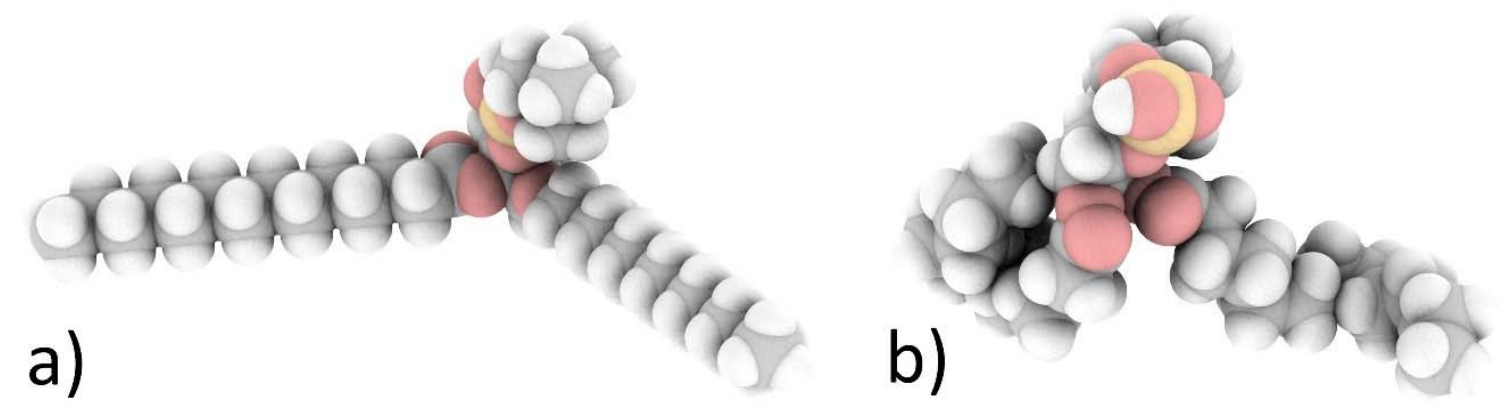

Figure 3.2 Space-filling models of DMPC molecule with explicit hydrogen atoms: a) The ideal conformation. b) A representative spatial arrangement of DMPC molecule in a bilayer. In both figures, gray atoms are carbon, white are hydrogen, red are oxygen and yellow is the phosphorus. The nitrogen atom is not visible.

Vesicles were then injected into the 1-stage microfluidic channel which was kept at around $1{ }^{\circ} \mathrm{C}$ with the signal generator set to the first harmonic frequency (Figure 3.3a). On actuation of the channel, all the vesicles were again observed to focus at the center nodal region of the channel (Figure 3.3b). In the next step, a slow sweep of temperature $\left(1^{\circ} \mathrm{C} \mathrm{min}^{-1}\right)$ was initiated while the applied frequency was simultaneously adjusted to accommodate the changes in the medium's properties with increasing temperature. Once the channel temperature reached $23.2^{\circ} \mathrm{C}$, the vesicles started to migrate to the anti-nodal regions (Figure 3.3c). It was mentioned earlier that hydrated 
PCs of medium-chain size like DMPC experience a series of thermotropic transitions between different lamellar phases. At a lipid-specific temperature called the pre-transition temperature $\left(T_{p}\right)$, the metastable or stable gel phase $\left(\mathrm{L}_{\beta^{\prime}}\right)$ undergo a transition to another gel phase known as the rippled phase $\left(\mathrm{P}_{\beta^{\prime}}\right)$ and on further increasing the temperature to a point known as the main transition temperature $\left(T_{m}\right)$, the $\mathrm{P}_{\beta^{\prime}}$ gel phase converts to a fluid (or liquid-disordered) phase $\left(\mathrm{L}_{\alpha}\right){ }^{69}$ It was also mentioned that lipid bilayers have been shown to display discernible differences in mechanical properties before and after these transitions..$^{91,124-126}$ DMPC vesicles, in particular, experience a dramatic decrease (by over an order of magnitude) in elastic compressibility moduli after the pretransition and eventually on melting from the ripple phase to the liquid-disordered phase $\left(\mathrm{L}_{\alpha}\right) \cdot{ }^{90,127}$ Therefore, as the temperature at which the node-to-antinode transition occurred for the DMPC system is between its $T_{p}$ at $14.3{ }^{\circ} \mathrm{C}$ and $T_{m}$ at $23.9^{\circ} \mathrm{C}$, ${ }^{128}$ it was concluded that the thermotropic changes in the membrane rendered the vesicles more compressible in the microfluidic channel such that they experienced a sign change in their $\phi$ and focused at the walls. Analogous to the lecithin system, DMPC GVs showed no repositioning from the walls on further increasing the temperature to more than $50{ }^{\circ} \mathrm{C}$. The GVs did migrate back to the center, however, on subsequent cooling $\left(-1{ }^{\circ} \mathrm{C} \min ^{-1}\right)$ at $22.5^{\circ} \mathrm{C}$, confirming a reversible thermo-acoustophoretic phenomenon in this system as well.
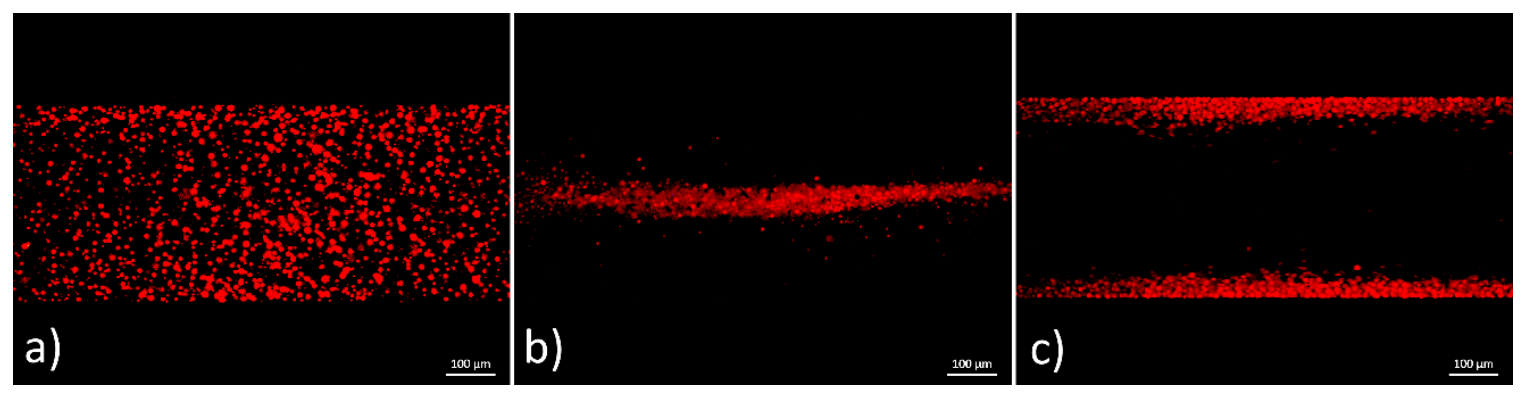

Figure 3.3 Thermo-acoustofluidic behavior of DMPC vesicles: a) at $1^{\circ} \mathrm{C}$ when the acoustic transducer is off. b) at the same temperature with the transducer on. c) at $23.2^{\circ} \mathrm{C}$, vesicles migrate towards the antinodes. (scale bar is $100 \mu \mathrm{m}$ ) 


\subsubsection{Thermo-Acoustophoretic Behavior of DPPC System}

DPPC is one of the most abundant lipids in human body and one of the most commonly studied lipids in biophysics and biochemistry. DPPC molecule, like DMPC, can be divided into two parts, the tail region containing the fatty acyl chains and the phosphocholine head region. The latter moiety carries a negative charge on its phosphate group and a positive charge on its quaternary ammonium group, giving rise to the hydrophilicity of the head region (Error! Reference source not found.). The fatty acyl chains are conversely nonpolar and hence hydrophobic. The calorimetrically determined $T_{p}$ and $T_{m}$ reported for DPPC are $34.2{ }^{\circ} \mathrm{C}$ and $41.4{ }^{\circ} \mathrm{C}$, respectively. ${ }^{128}$ It is therefore expected to observe the node-to-antinode transition occur at some point between these two temperatures. It is worth mentioning, however, that the values reported here as the transition temperatures may not be the true equilibrium transition temperatures, given that transitions involving the lipid chain melting may be delayed in some instances as the transition is a kinetically limited process.
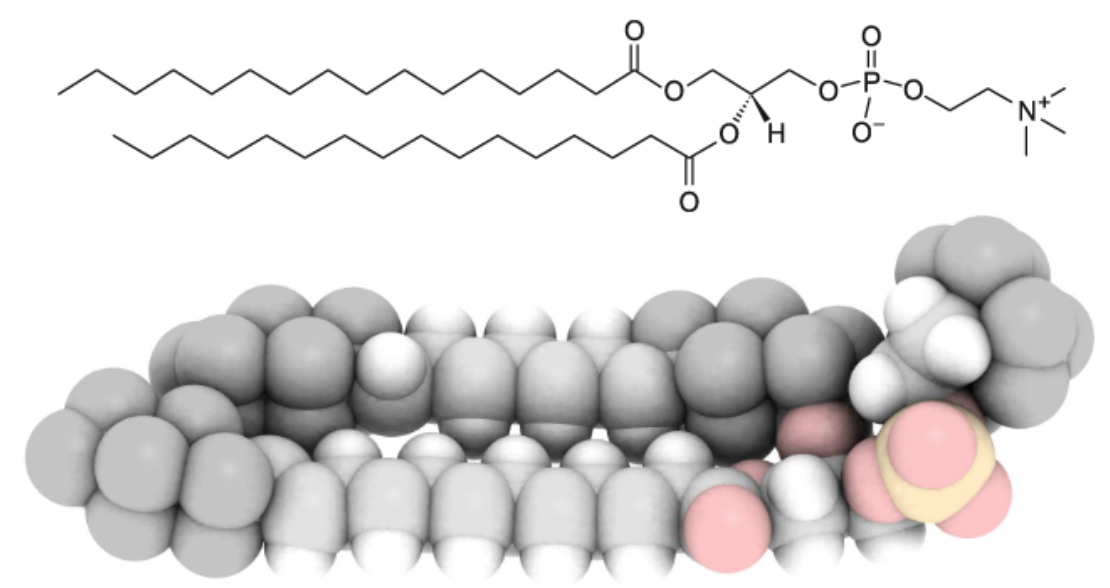

Figure 3.4 The skeletal and space-filling molecular models of DPPC showing the phosphocholine headgroup on the right side and the fatty acyl chains extended towards the left side. Both fatty acyl chains are saturated and nonpolar, comprising the hydrophobic moiety of the molecule. The phosphocholine molecule is on the other hand polar and therefore hydrophile. 
In order to investigate the thermo-acoustofluidic properties of DPPC system, giant vesicles prepared from pure DPPC and tagged with Nile red were injected into the 1-stage microfluidic channel which was kept at $1{ }^{\circ} \mathrm{C}$. Before actuation of the channel, vesicles were randomly dispersed in the channel. On actuation of the channel, however, the same nodal focusing behavior was observed for the DPPC system. The nodal focusing was accomplished in less than 3 seconds for most of the vesicles. To investigate the existence of a node-to-antinode transition point, like what observed for the DMPC system, a sweep of temperature $\left(1^{\circ} \mathrm{C} \min ^{-1}\right)$ was then initiated while the vesicles were monitored under the fluorescent microscope. As the temperature exceeded $37.6^{\circ} \mathrm{C}$, GVs collectively migrated towards the antinodes located at the walls in less than 30 seconds. Furthermore, analogous to the other systems studied, they remained at the walls when the temperature was raised to around $50{ }^{\circ} \mathrm{C}$. Figure 3.5 shows the time-lapse micrographs of this transition as the temperature exceeded $37.6^{\circ} \mathrm{C}$. Of significance is also that the transition observed is happened at a clear-cut point, implying that vesicle with homogeneous composition, irrespective of their size, experiences the transition at the same point.

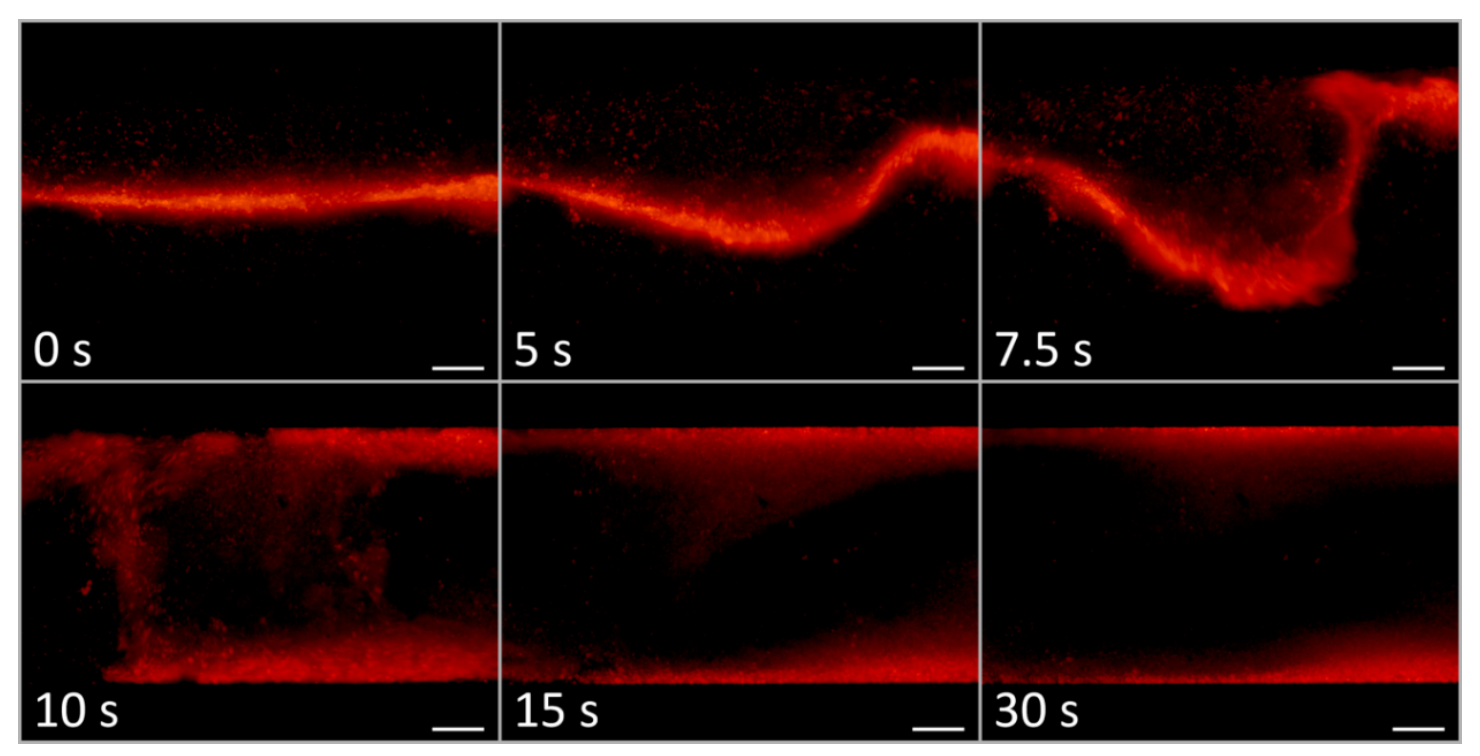

Figure 3.5 Time-lapse fluorescence micrographs of the transition from nodal focusing to anti-nodal focusing of DPPC vesicles as the temperature exceeded $37.6{ }^{\circ} \mathrm{C}$ at time $t=0 \mathrm{~s}$. The focusing was complete at time $t=30 \mathrm{~s}$. 
In order to better understand the physical basis of the observed node-to-antinode transitions, the temperature-dependent compressibility data of the DMPC and DPPC vesicles were extracted from the literature to calculate the changes of $\phi$ with temperature. Figure 3.6 shows the temperature dependence of the relative value of $\phi$ for the DMPC and DPPC system. As can be seen, for both systems, $\phi$ exhibits a decreasing trend with increasing temperature until it reaches a minimum point at a temperature very close to the $T_{m}$ of the respective system. After that point, $\phi$ would again increase until it reaches a peak followed by a small decrease and a stabilization after that. This is in agreement with the observed changes in the bending rigidity of DMPC and DPPC bilayers with temperature, beginning with a sharp decrease in the gel phase at temperatures close to the $T_{m}$ of the lipid, followed by an increase in the fluid phase after the $T_{m}$ and then a small decrease between $T_{m}+3$ and $T_{m}+6$, ending with a stabilization at temperatures above $T_{m}+6 .{ }^{67,89,90,129}$ As can be seen in Figure 3.6, increasing temperature well above the $T_{m}$ (around $50{ }^{\circ} \mathrm{C}$ in this case) shows that the total regain in the bending rigidity (or decrease in compressibility) after the main drop is nevertheless not enough to switch the $\phi$ sign to a positive value and therefore vesicles remain at the walls.

Of significance is also the point at which $\phi$ becomes zero. This point, which does not necessarily correspond to the $T_{m}$ of the lipid, is found to occur somewhere between the $T_{p}$ and $T_{m}$ of the respective system; in the case of DMPC closer to the $T_{m}$ but for DPPC almost in the midpoint. This lipid-specific point at which vesicles switch their focusing on assuming an opposite-sign $\phi$ was dubbed by us the "acoustic contrast transition temperature" or " $T_{\phi}$ ". The acoustic contrast transition temperature, or simply the acoustic contrast temperature, to be precise, is a point at which the net acoustic radiation force on the vesicles becomes zero, hence there exist no preferred location for the vesicle to focus. From Figure 3.6, the $T_{\phi}$ for the DMPC system can be found on the temperature axis at the left edge of the green bar and for the DPPC system at the right edge. 


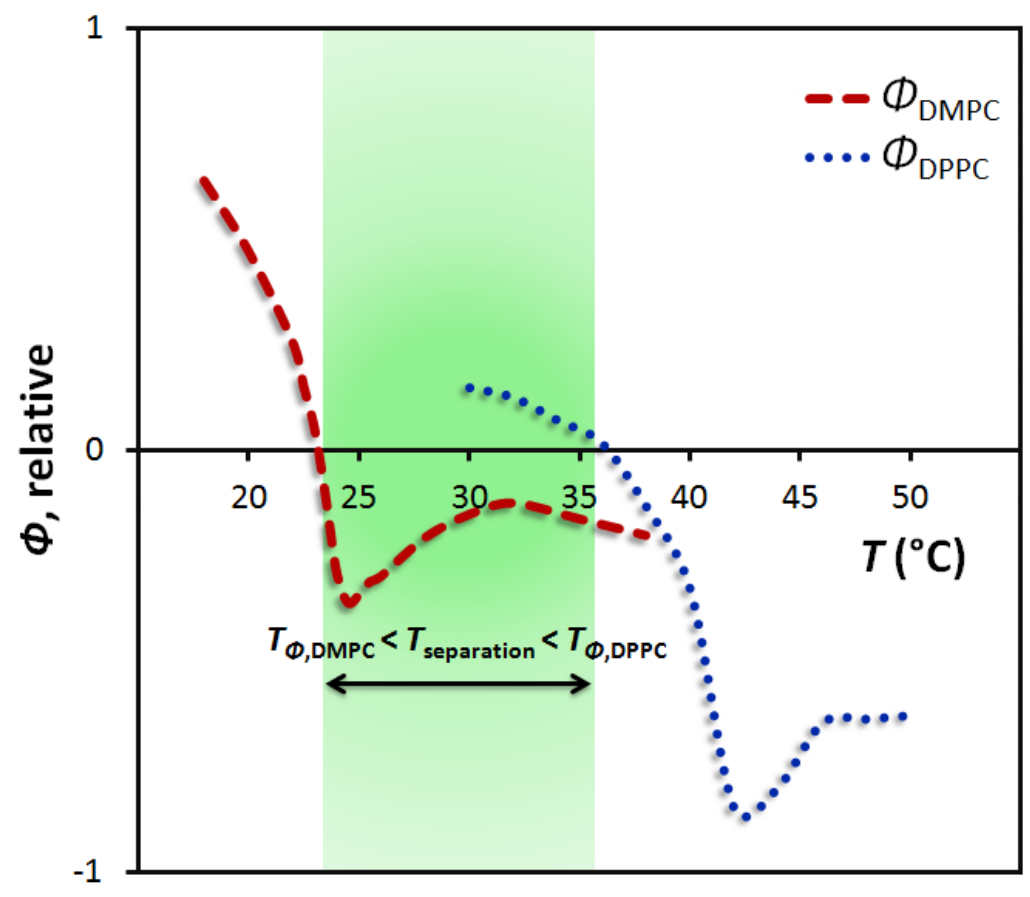

Figure 3.6 The relative values of $\phi$ for DMPC and DPPC vesicles vs. temperature; calculated (Appendix IV) using vesicle compressibility data from the literature. ${ }^{130,131} \boldsymbol{\phi}>\boldsymbol{0}$ indicates nodal focusing while $\phi<0$ indicates anti-nodal focusing. On the temperature axis where $\phi=0$, is the point dubbed by us the acoustic contrast temperature or $T_{\phi}$. With $T_{\phi \text {,DMPC }}<T_{\text {separation }}<T_{\phi \text {,DPPC, opposite }}$ focusing is achieved.

Also, evident from Figure 3.6 is temperature range (shown as a green bar) within which DMPC vesicles have assumed a negative $\phi$, while DPPC vesicles still exhibit a positive $\phi$. The existence of this temperature window offered us the possibility of separating these vesicles, given the fact that they display different focusing behaviors in that window. Separation studies are discussed in more detail in Section 3.4.

One could also experimentally obtain the average velocity of the migration using timelapse micrographs like the one in Figure 3.5. In this figure, the average transversal velocity was approximately $15 \mu \mathrm{m} \cdot \mathrm{s}^{-1}$, which was mainly dependent on the magnitude and sign of $\phi$, the distance from the wall, and vesicles' diameter. The magnitude of $\phi$ affects the velocity of the migration as the net force acting on the vesicles is proportional to $\phi$. In the transversal direction, forces acting 
on the vesicles include the acoustic radiation force and the Stokes' drag force. Therefore, by increasing the temperature, the net force increases due to larger $\phi$ and lower drag force due to lower viscosity of the medium.

It was mentioned earlier that in all experiments the transducer was set to actuate the channel at the first harmonic frequency. This frequency was determined for the microfluidic device using initial conditions. Since the speed of sound in the bulk phase is however dependent on temperature, substantial temperature increase required adjustments in the frequency to accommodate this change. While this adjustment maintained denser vesicle focusing at both the nodal and anti-nodal zones, it did not change the overall behavior of the vesicles, and was deemed optional. The effect of the medium's property change on $\phi$ due to heating/cooling is assessed in Figure 3.7. By comparing scenarios of (a) only properties of vesicles change (fixed values for room temperature conditions) versus (b) only properties of medium change due to the $T$ change (fixed values for room temperature conditions), it was observed that the reversal of the theoretical $\phi$ was predominantly dependent on the compressibility of the vesicles. Similar observations were also obtained for DMPC vesicles suspensions.
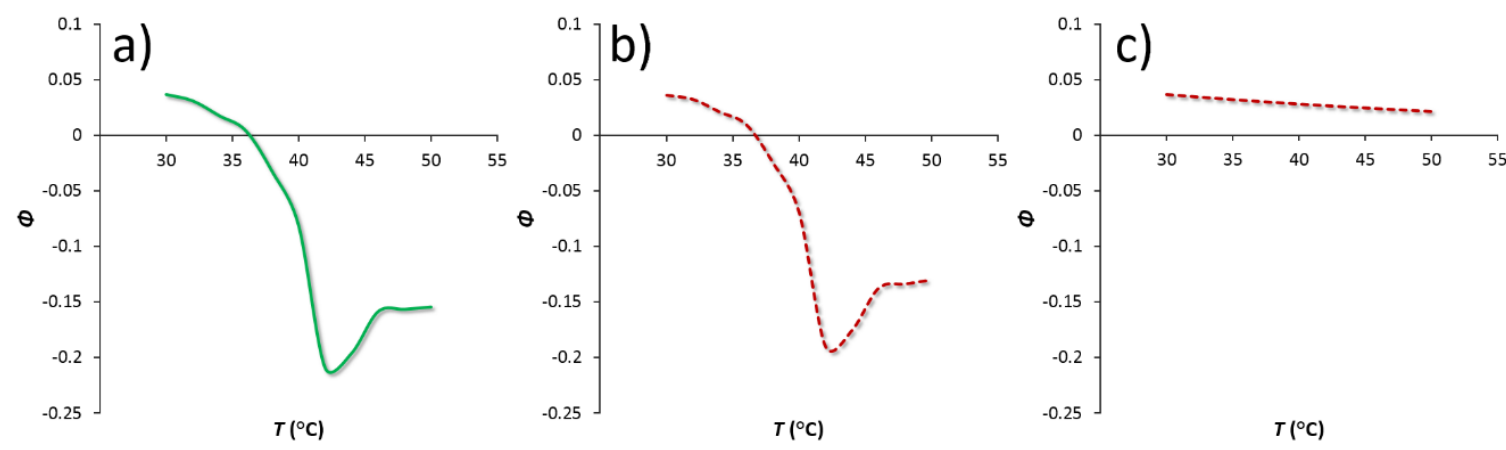

Figure 3.7 Change of $\phi$ with temperature for (a) DPPC vesicles, (b) considering a scenario where the adiabatic compressibility of the bulk medium retains room temperature value at various temperatures, and (c) considering a scenario where the adiabatic compressibility of the vesicles retains room temperature values at various temperatures. 
Like lecithin and DMPC, the temperature recorded as the $T_{\phi}$ in the cooling experiment was lower by 1 degree than the $T_{\phi}$ measured in the heating experiment, presumably due to the heat capacity in the vesicles. This temperature gap between the transitions was however minimized by slowing down the temperature sweep.

\subsubsection{Thermo-Acoustophoretic Behavior of POPC System}

Among the most commonly studied PCs is 1-palmitoyl-2-oleoyl-sn-glycero-3phosphocholine or simply POPC with the saturated palmitic acid chain in the $s n-1$ position and the monounsaturated oleic acid chain in the $s n-2$ position. POPC, in contrast to DMPC and DPPC, has its solid phase directly convert to the fluid phase at around $-3{ }^{\circ} \mathrm{C}$ in excess water conditions $\left(\mathrm{L}_{\beta} \rightarrow\right.$ $\left.\mathrm{L}_{\alpha}\right){ }^{69}$ which is relatively lower than the reported calorimetrically determined transition temperatures for DMPC and DPPC. Why POPC, despite having a longer acyl chain, has a lower transition temperature is related to its unsaturated double bond which give rise to a kink in the oleic acid chain, creating an extra space in the bilayer. These extra spaces in the bilayer, which disrupt the regular periodic order of the membrane, give rise to a weaker van der Walls interactions and therefore lower transition temperature for the bilayer.
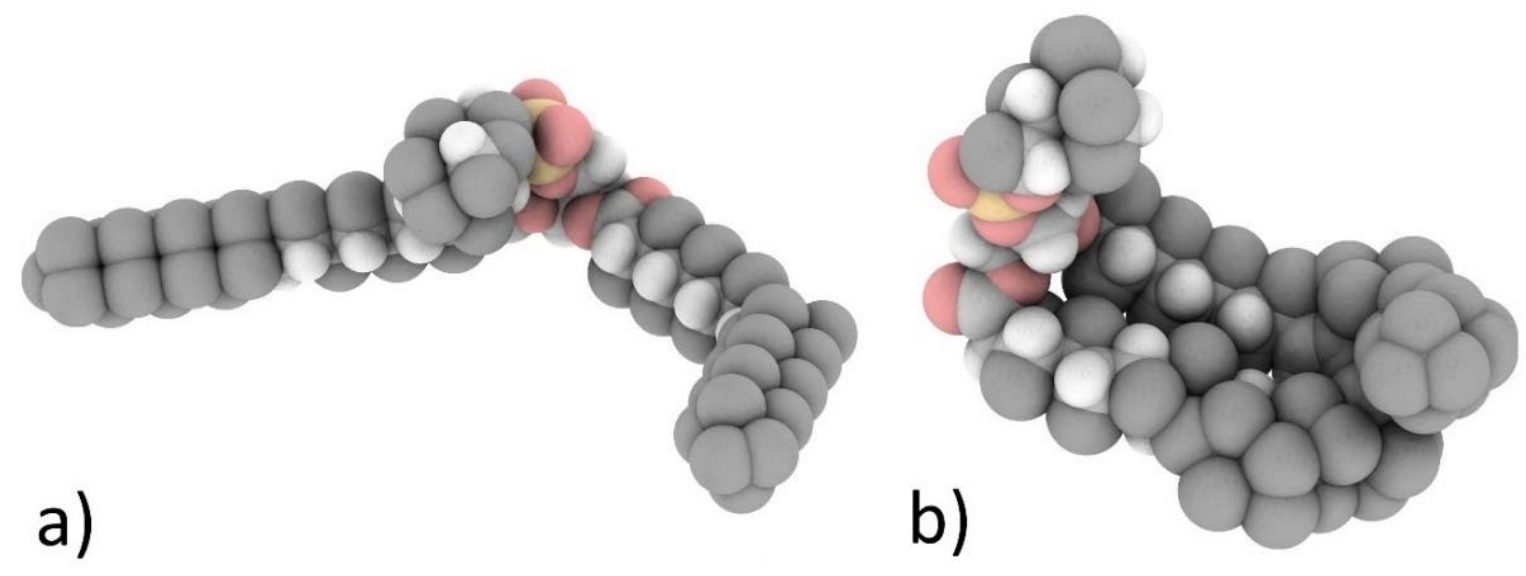

Figure 3.8 Space-filling models of POPC molecule with explicit hydrogen atoms: a) The ideal conformation. The kink on the right chain is due to the double bond of the oleic acid. b) A representative spatial arrangement of POPC molecule in a bilayer. 
To examine the thermo-acoustofluidic behavior of POPC system, giant vesicles prepared from pure POPC and tagged with Nile red were injected into the 1-stage microfluidic channel which was kept at $1{ }^{\circ} \mathrm{C}$. Like the other systems, vesicles were randomly dispersed in the channel before actuation of the channel. On actuation of the channel, however, the same nodal focusing behavior was observed for the vesicles. The nodal focusing was quick and accomplished in less than 3 seconds. In the next step, a sweep of temperature $\left(1^{\circ} \mathrm{C} \min ^{-1}\right)$ was initiated while the vesicles were monitored under the microscope. As the temperature exceeded $26.5^{\circ} \mathrm{C}$, vesicle collectively migrated towards the antinodes and, like the other systems, they remained at the walls even after the temperature was raised to over $50{ }^{\circ} \mathrm{C}$, confirming that for this system only point can be identified as the $T_{\phi}$. In this case, however, the $T_{\phi}$ recorded is higher than the $T_{m}$ reported for the system. This could be explained by the fact that the $T_{\phi}$, as was shown in Figure 3.6, does not necessarily correspond to a specific transition point in the membrane, but it is indeed a direct outcome of the thermotropic transition(s) in the membrane, rendering the membrane less rigid or more compressible. This is more evident in Figure 3.9, which illustrates a POPC bilayer membrane in gel and fluid states constructed using the coordinates data from molecular dynamic simulations provided by Heller. ${ }^{132,133}$ It is clear that the main transition from the ordered state to the disordered state the lateral crystalline arrangement and chain order of the lipid molecules are lost. Changes in volume and area up to $4 \%$ and $25 \%$, respectively, ${ }^{134}$ which in turn leads to changes in membrane curvature, bring about considerable changes in thermoelastic properties of the membrane such as bending rigidity and compressibility. ${ }^{134}$ The $T_{\Phi}$ of vesicles however occurs at a specific point during this transition or after it, as $T_{\Phi}$ in addition to its dependence on the composition of the vesicle, it is also dependent on the medium's composition and properties such as salt concentration, $\mathrm{pH}$, and density. This was earlier demonstrated by Eq. 11, which shows how $\phi$ in addition to the density and compressibility of vesicles, is also dependent on the density and compressibility of the medium. In vesicles, however, since the internal volume of the vesicle is extremely large compared to its 
enclosing membrane, it is safe to assume that the density ratio is very close to unity. Therefore, $\phi$ can be mainly assumed to be dependent on the compressibility ratio of the vesicles and medium. Therefore, since the changes of the compressibility of the medium (in this case water) has a competing effect, the point at which the compressibility ratio becomes unity does not necessarily correspond to the $T_{m}$ of the bilayer.

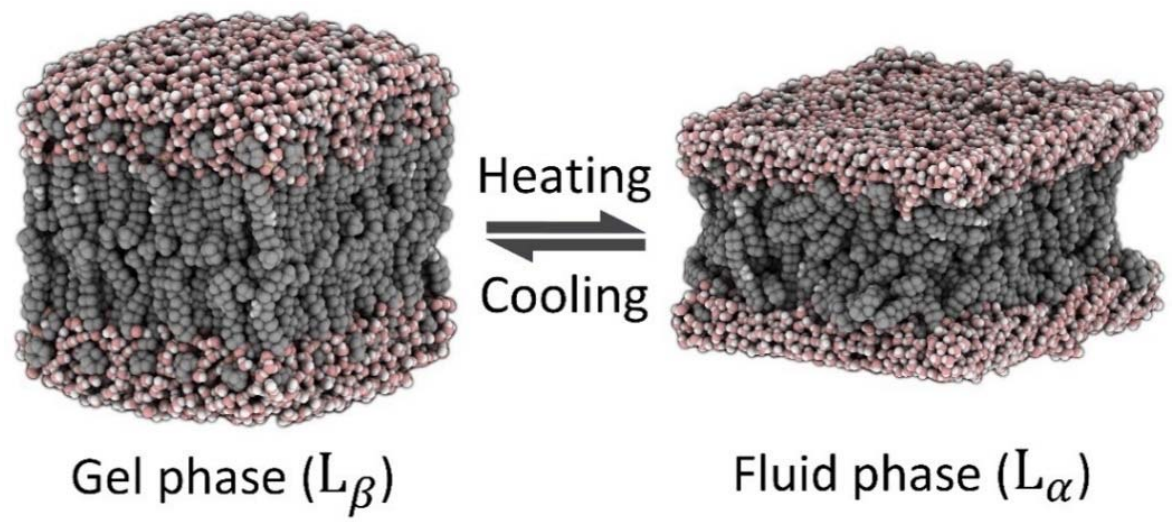

Figure 3.9 Schematic illustration of a POPC bilayer membrane in gel (ordered) and fluid (disordered) states. During the main transition from the ordered state to the disordered state the lateral crystalline arrangement and chain order of the lipid molecules are lost. (The bilayer is surrounded on both sides by water molecules shown in pink and white. The gray atoms are the hydrogen atoms explicitly shown on the acyl chains).

\subsection{Thermo-Acoustophoretic Behavior of Vesicle with Binary Compositions}

To further demonstrate the capability of thermo-acoustophoresis, vesicles with binary compositions were prepared using various molar ratios of DMPC and DPPC. To investigate the existence of $T_{\phi}$ for the binary compositions, GVs tagged with Nile red were injected into the 1stage microfluidic channel and were investigated for both heating and cooling transitions both at 1 ${ }^{\circ} \mathrm{C} \min ^{-1}$. In another series of experiments, the heating and cooling rate was set to $5{ }^{\circ} \mathrm{C} \min ^{-1}$ to examine the effect of heating/cooling rate on the difference between the $T_{\phi}$ measured in heating and the one measured during cooling. Figure 3.10 presents the results obtained from the measurement of $T_{\phi}$ for different binary compositions, namely 100:0, 85:15, 70:30, 50:50, 30:70, 15:85, and 0:100 DMPC:DPPC in experiments with heating and cooling rate of $5{ }^{\circ} \mathrm{C} \mathrm{min}^{-1}$. The 
measured points are superimposed on the isomorphous phase diagram of the DMPC:DPPC lipid binary mixture, drawn using the data obtained from the mean value of the theoretical data and the experimental data from electron spin resonance (ESR) and differential scanning calorimetry (DSC) studies. ${ }^{135,136}$ It is interesting to mention that for all the binary composition examined here a single $T$ can be identified as the $T_{\phi}$ of the system. There exists however a difference between the temperature measured in the heating and the one measured in the cooling, but this difference, which is presumably due to the heat capacity in the GVs, was minimized to less than 1.5 degrees or even eliminated by slowing down the cooling rate to $1^{\circ} \mathrm{C} \mathrm{min}^{-1}$. Also of significance is the trend of the changes of $T_{\phi}$ which is observed to occur at approximately the solidus line for all the compositions, right before the transition from ripple to liquid phase occur. This is in agreement with what was observed for the single component DMPC and DPPC systems.

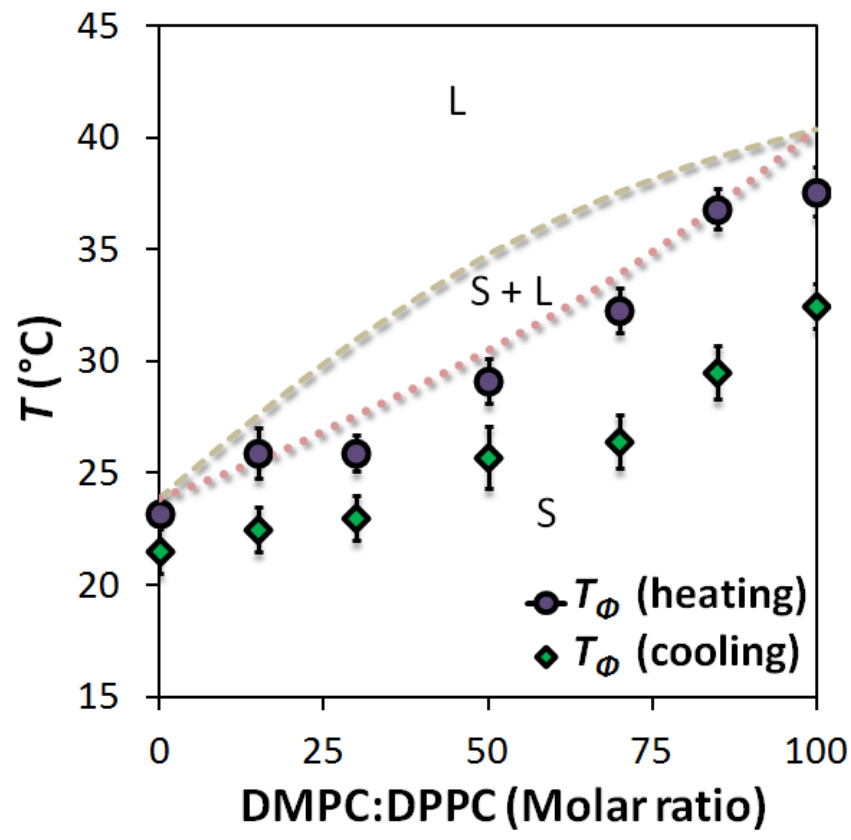

Figure 3.10 The $T_{\phi}$ of DMPC:DPPC binary mixtures superimposed on the thermotropic isomorphous phase diagram of the same system drawn using the data obtained from the mean value of the theoretical and experimental data obtained by electron spin resonance (ESR) and differential scanning calorimetry (DSC) studies (with solidus (dotted) and liquidus (dashed) curves). ${ }^{135,136}$ The violet circle markers denote the measured $T_{\phi}$ during the heating experiment while the green diamond markers denote the measured $T_{\phi}$ during the cooling curve. The heating $T_{\phi}$ nearly overlapped with the solidus line over the molar ratio range. The error bars represent the maximum and minimum $T_{\phi}$ observed. 


\subsection{Thermo-Acoustophoretic Separation}

The first samples chosen for the separation studies were 100:0 and 70:30 DMPC:DPPC samples. The $\Delta T_{\phi}$ for these two vesicles was $2.7{ }^{\circ} \mathrm{C}$ (pure DMPC with $T_{\phi}=23.2{ }^{\circ} \mathrm{C}$ and $70: 30$ DMPC:DPPC $T_{\phi}=25.9^{\circ} \mathrm{C}$ ). The separation temperature was selected as the approximate mid-point of $24.5{ }^{\circ} \mathrm{C}$. At this separation temperature, the mixture of vesicles was shown to be randomly dispersed in a channel that was not acoustically excited (Figure 3.11a). However, once the transducer was switched on $\left(f=1.40 \mathrm{MHz}\right.$ and $\left.50 \mathrm{~V}_{p p}\right)$, the separation of vesicles promptly started and completed in less than 30 seconds. The distribution of vesicles can be seen in Figure $3.11 \mathrm{~b}$ with red-stained 100:0 DMPC:DPPC at the walls and the blue-stained 70:30 DMPC:DPPC at the center of the channel.
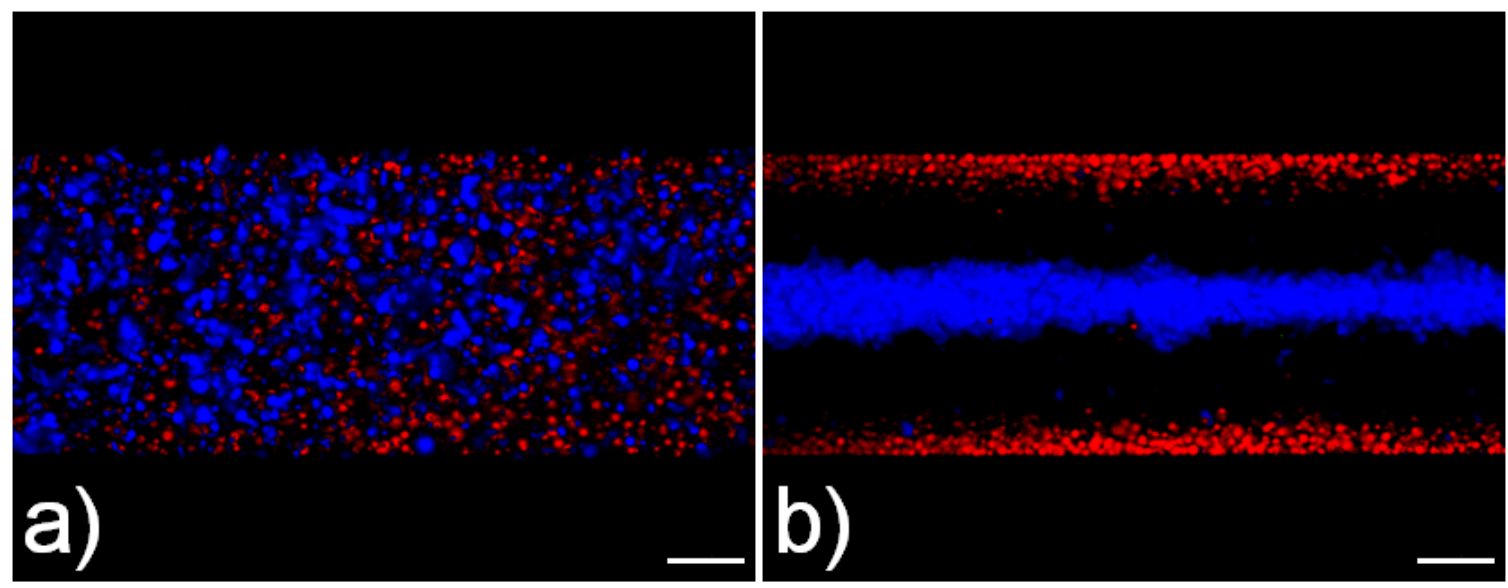

Figure 3.11 Monitoring the performance of the separation experiment of 100:0 DMPC:DPPC and 70:30 DMPC:DPPC samples. (a) Optical fluorescence micrographs of 100:0 (red) and 70:30 (blue) DMPC:DPPC vesicles dispersed in the microchannel at $24.5^{\circ} \mathrm{C}$ with the acoustic transducer switched off. (b) The same vesicles after 3 seconds of the transducer switched on showing 100:0 DMPC:DPPC completed migration towards the pressure antinodes (red) at the walls and 70:30 DMPC:DPPC completed migration towards the pressure node (blue) in the center (scale bars in both a and $b$ images are $150 \mu \mathrm{m})$.

The distribution of the vesicles in the channel was quantified using averaged fluorescent intensity in the direction normal to the flow and the relative intensities are shown in Figure 3.12a. It is evident from the picture that even for a system with a small $\Delta T_{\phi}$ a separation of high-efficiency 
can be achieved provided the separation temperature is selected at the midpoint of the temperature window. The purity of each of the vesicle systems recovered from the outlets were 93-97\%. Further improvement of the separation can be achieved by optimizing variables such as volumetric flow rate, separation temperature, and the amplitude of the ultrasonic standing wave adjusted by the power generator.

The effect of the separation temperature of the vesicles mixture was also investigated for separation of 100:0 DMPC:DPPC and 0:100 DMPC:DPPC systems. Separation experiments were conducted over different separation temperatures ranging from $17-43{ }^{\circ} \mathrm{C}$ while the separation window, i.e. where $T_{\phi \text {,DMPC }}<T_{\text {separation }}<T_{\phi \text {,DPPC }}$, was determined to be $23.2-37.6{ }^{\circ} \mathrm{C}$. Figure 3.12 presents the calculated target outlet purities at 6 different temperatures over the allowable separation window for these two systems. At a temperature $\left(23.6{ }^{\circ} \mathrm{C}\right)$ slightly higher than the $T_{\phi}$ for the DMPC system $\left(23.2{ }^{\circ} \mathrm{C}\right)$, the purities were $92 \%$ and $93 \%$ for DMPC and DPPC, respectively. This was due to the fact that right after the transition temperature $\phi$ would not have reached a large enough value to produce high acoustic radiation force. In this case, some smaller vesicles, not hydrodynamically big enough, may migrate slower. When the separation temperature was chosen closer to the midpoint of the two $T_{\phi}$ values $\left(29^{\circ} \mathrm{C}\right)$, the purities were observed to improve to $98 \%$ for both DMPC and DPPC systems. The deviation from the mean value also decreases at higher temperature, indicating a more reliable separation at the midpoint. At a temperature right below the $T_{\phi}$ of the DPPC system $\left(37.2{ }^{\circ} \mathrm{C}\right)$, purities of $96 \%$ and $95 \%$ were obtained for DMPC and DPPC, respectively. This drop from the higher purity for the midpoint could also be explained by the lower $\phi$ values for the DPPC vesicles. However, the purities for both outlets were improved compared to $T=23.6{ }^{\circ} \mathrm{C}$ due to the reduced drag force of the medium at higher temperatures. 


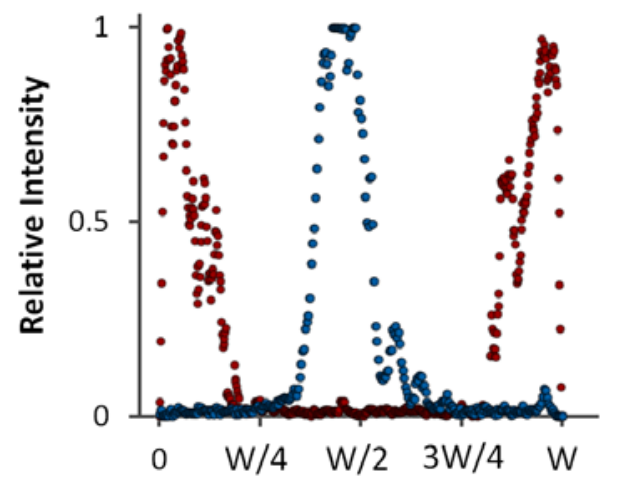

a)

Distance (Width of the channel)

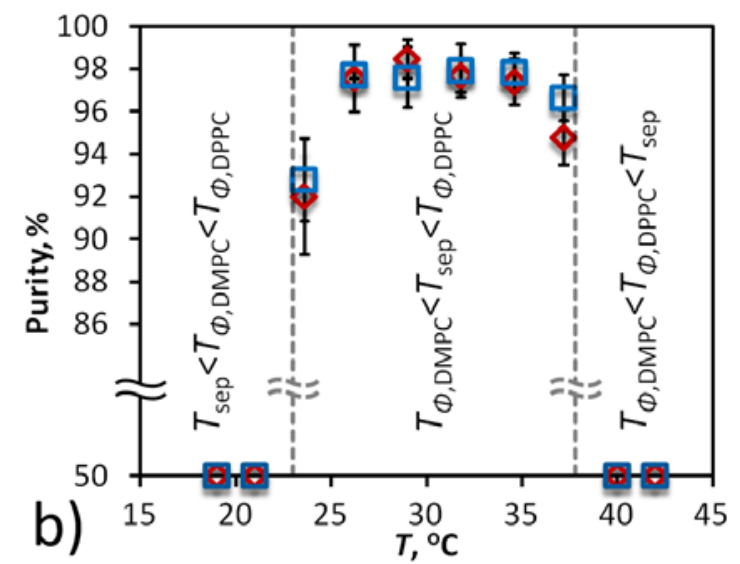

Figure 3.12 Performance of the separation of DMPC from 70:30 and 100:0 DMPC:DPPC: a) Relative fluorescence intensity scanned in the direction normal to the flow for both red and blue emissions in the separation experiment of 100:0 DMPC:DPPC and 70:30 DMPC:DPPC samples. The separation temperature was $25^{\circ} \mathrm{C}$, the approximate midpoint of the allowable window. b) Target outlet purities at 6 different temperatures over the allowable separation window $\left(T_{\phi, D M P C}<T_{\text {separation }}<T_{\phi \text {,DPPC }}\right.$ ) for pure DMPC and DPPC vesicle systems. The error bars represent the standard deviation for $n=3$.

To demonstrate the thermo-acoustophoretic separation for a system composed of two vesicle types with binary compositions, two vesicle samples were chosen with 50:50 and 30:70 DMPC:DPPC compositions. The previously determined $T_{\phi}$ for these systems was $29{ }^{\circ} \mathrm{C}$ and 32 ${ }^{\circ} \mathrm{C}$, respectively. To achieve the best separation efficiency the approximate midpoint of the temperature window between these two temperatures was chosen as the separation temperature, which was kept constant in all the experiments. After adjusting the channel temperature at $30.7^{\circ} \mathrm{C}$, GVs mixed at the ratio of 50/50 were infused into the channel and randomly disperse in the channel. Figure 3.13a shows an optical micrograph of the dispersed GVs upon entry into the channel at 30.7 ${ }^{\circ} \mathrm{C}$ with the piezoelectric transducer switched off. After the transducer switched on, 50:50 DMPC:DPPC GVs made a swift migration towards the pressure antinodes at the walls and 30:70 DMPC:DPPC GVs gathered at the center node of the channel. This was expected as the $T_{\text {separation }}$ is greater than the $T_{\phi}$ for the 50:50 DMPC:DPPC GVs and is less than the $T_{\phi}$ for the 30:70 DMPC:DPPC system. Figure $3.13 \mathrm{~b}$ shows the $\mathrm{GVs}$ at $30.7^{\circ} \mathrm{C}$ after the piezoelectric transducer was switched on. The distribution of the GVs in the channel was quantified using averaged 
fluorescent intensity in the direction normal to the flow and the relative intensities are shown in Figure 3.13c. It is evident again from this picture that a high-efficiency separation is realized through running the device at the midpoint of the temperature window. The average purity of the collected GVs at the target outlets was $93 \%$ for outlet 1 and $95 \%$ for outlet 2 . Figure $3.13 \mathrm{~d}$ shows the expected outlet for the collection of 50:50 and 70:30 DMPC:DPPC GVs after separation. It was observed that further improvement of the separation can be achieved by increasing volumetric flow rate from $5 \mu 1 \mathrm{~min}^{-1}$ to $10 \mu 1 \mathrm{~min}^{-1} .{ }^{137}$ The amplitude of the acoustic radiation is another parameter, upon increasing which a more powerful focusing could be achieved in the channel.
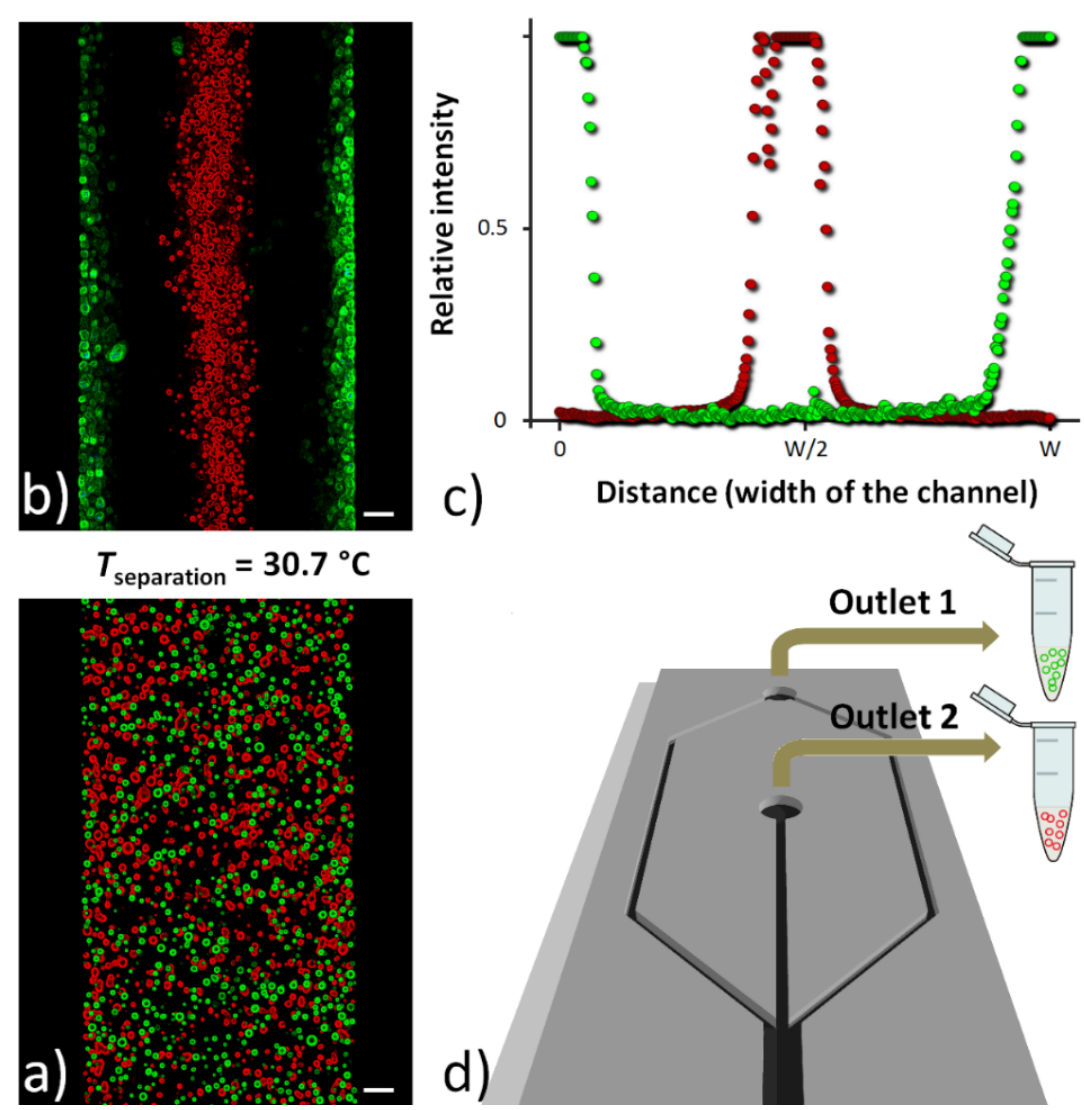

Figure 3.13 Separation of 50:50 DMPC:DPPC (green) from 30:70 DMPC:DPPC (red) sample: a) Optical micrographs of dispersed pure 50:50 DMPC:DPPC and 30:70 DMPC:DPPC GVs at $30.7^{\circ} \mathrm{C}$ with the piezoelectric transducer off. $b$ ) The same vesicles at the same temperature after the transducer switched on showing 50:50 DMPC:DPPC GVs pushed towards the antinodes and 30:70 DMPC:DPPC GVs gathered at the center node (scale bars are $80 \mu \mathrm{m}$ ). c) Performance of the separation at the midpoint temperature shown as the fluorescence intensity scanned in the transversal direction of the channel. d) Target outlet of GVs, based on which the purity of collected vesicles was calculated. 


\subsection{Conclusion}

In summary, we have demonstrated the possibility of separating vesicles based on their membrane composition using thermally-assisted acoustophoresis in a microfluidic device. This technique allowed us to exploit the unique acoustic contrast temperatures of vesicles to yield a separation at $T_{\phi, 1}<T_{\text {separation }}<T_{\phi, 2}$. Under this separation temperature condition, vesicles migrated in opposite directions, towards the central region or the wall regions of the channel. The transition temperature observed in this study, $T_{\phi}$, occurred at a temperature $T_{p}<T_{\phi}<T_{m}$ indicative of a mechanical transition in the membrane property, i.e., membrane compressibility, rather than a distinct phase transition. This observation was corroborated using compressibility data to calculate the $T_{\phi}$ values, which closely matched the $T_{\phi}$ measurements obtained in this study. Therefore, we concluded that due to thermotropic phase transitions, the vesicles had an apparent effect on their mechanical properties, especially compressibility. This effect provided tunability of $\phi$ depending on temperature, thus enabling the existence of a temperature range in which opposite $\phi$ signs existed. Within this temperature "window" the vesicles become mechanically distinct and thus differentiable in the acoustic radiation field, yielding to their separation. High separation efficiencies were achieved and we anticipate that it can be further improved in the future. While this method is applicable to nearly all sorts of lipid vesicles, we also recognize that it may pose harm to cells if applied at temperatures exceeding $43{ }^{\circ} \mathrm{C}$ for extended periods of time. ${ }^{138} \mathrm{We}$ envision that this separation technique can prove to be of value as a stand-alone or in junction with other separation methods that can yield medical separations and diagnostics, especially in cancers, infections, and red blood cell anemia research and also in the separation of extracellular vesicles such as exosomes and microvesicles. 


\section{Thermo-Acoustofluidic Separation of Cholesterol Containing Vesicles}

\subsection{Introduction}

In vesicles and cells, the membrane draws on a combination of physiochemical properties that allow interactions with its surroundings while maintaining the two environments separate. ${ }^{66}$ Among the important properties of membranes are mechanical properties such as fluidity, or its inversely proportional property, stiffness. Membrane fluidity is linked to the mobility of biomolecules within the membrane and their ability to rotate. ${ }^{139}$ The stiffness of the membrane has been reported to determine the ability of the vesicle or cell to perform its function whenever cell deformability is necessary. ${ }^{140,141}$ Various pathological conditions have been associated with changes in the cellular stiffness. ${ }^{109}$ Some of these conditions include cancers, ${ }^{142-145}$ parasitic infections, ${ }^{110}$ and red blood cell anemia. ${ }^{111}$

Standing-wave acoustophoresis draws on attributes such as non-destructiveness, label-free operation, high separation efficiency and throughput, and good size resolution. ${ }^{53}$ Moreover, its unique separation criteria, i.e. density and compressibility make acoustophoresis a desirable method for the separation of cells. In an ultrasonic standing-wave, the migration direction of a suspended particle is determined by the sign of the acoustic contrast factor, $\phi,{ }^{11}$ which in addition to being dependent on the density of the particle and the medium, is also dependent on the compressibilities of the particle and medium. As mentioned earlier, positive $\phi$ affords nodal migration and negative $\phi$ affords anti-nodal migration for the suspended particle. With changing temperature, the value of $\phi$ for a suspended particle changes due to the changes in the density of the particle and medium and also in the compressibility of the particle and medium. We previously demonstrated that $\phi$ for a vesicle with known membrane composition, at a given temperature, can be determined based on the density and compressibility of the particle and medium at that 
temperature. ${ }^{123}$ The density ratio in $\phi$ is however very close to unity and therefore the compressibility ratio becomes the determining factor. ${ }^{123}$

The potential of acoustophoresis to assume a significant role in stiffness-based separations is a major impetus for research in this field. Cells, however, in most cases have displayed positive $\phi$ at normal body temperatures. ${ }^{55}$ Therefore, simple alterations are required to overcome the differentiation issue in cell-from-cell separations. A number of initiatives have been undertaken, such as adding density-mediating agents, ${ }^{43}$ multi-node separation based on size, ${ }^{27}$ and binding cells to elastomeric particles. ${ }^{62}$ Recently, we introduced temperature as a new parameter to harness the temperature-dependent changes in the mechanical properties of phospholipid membranes. ${ }^{123}$ The chip integrated a concurrent application of piezoelectric and thermoelectric effects on a singlestream flow in a microchannel. As vesicles passed through the ultrasonic radiation field kept at a predetermined temperature, they experienced a stiffness-dependent force leading to their migrations either towards the node or the anti-nodes, determined by the sign of $\phi$. Therefore, for a system composed of two types of vesicles with distinct cellular stiffness values, the thermotropic sign change of $\phi$ enables the existence of a temperature "window" within which opposite $\phi$ signs exist. As ultrasonic standing-waves are intrinsically capable of accommodating subtle differences in compressibility, if the device temperature is set within that window, vesicles were separated at efficiencies exceeding $95 \%{ }^{123}$

Also of interest for separation are the different types of extracellular vesicles (EVs), such as exosomes, micro-vesicles (MVs), apoptotic bodies, and large oncosomes with membrane compositions inherited from their parent cells. ${ }^{146,147}$ These vesicles which are often isolated through cumbersome and time-consuming methodologies from biological fluids such as blood, urine, saliva, and others have been identified as carriers of biomarkers for cancers, ${ }^{148}$ infectious, ${ }^{149}$ and neurodegenerative diseases. ${ }^{150}$ Therefore, the development of a quick and gentle separation method 
that is capable of sorting extracellular vesicles based on their composition, i.e. cell of origin, is of great biomedical importance.

In this chapter, we first examine the influence of membrane composition, particularly cholesterol content $\left(X_{\mathrm{chol}}\right)$ on the thermo-acoustophoretic behavior of vesicles. Vesicles of binary and ternary compositions of different phospholipids and cholesterol were prepared and examined

for their thermo-acoustophoretic behaviors. ${ }^{151}$ The dependence of $\phi$ on temperature, dependence of $T_{\phi}$ on cholesterol content, and the efficiency of the separations at various $T_{\text {separation }}$ were studied to establish the capability and performance of thermally-assisted acoustophoresis (thermoacoustophoresis) for cholesterol-containing vesicles.

\subsection{Cholesterol}

\subsubsection{Cholesterol as a Molecule}

In mammalian cells, cholesterol content plays a determining role in the membrane's stiffness. ${ }^{152}$ Cholesterol is an essential component to the proper function of cellular membranes and is either produced by the cells themselves or obtained through diet. ${ }^{65}$ The cholesterol molecules do not form self-assembled bilayers like other lipids but rather form nonpolar crystals. ${ }^{152}$ The cholesterol molecule's large planar ring structure is attached to one polar hydroxyl group on one side and to a short nonpolar hydrocarbon chain on its other side. ${ }^{64}$ Because of this rigid ring structure, cholesterol generally increases the order parameter when introduced to lipid membranes at physiological conditions by organizing the molecular structure of the membrane. ${ }^{152}$ However, when added at lower than physiological temperatures, cholesterol tends to increase the membrane fluidity as its large ring structure disrupts the hydrophobic portion of the phospholipid bilayers. ${ }^{63}$ At higher than physiological temperatures, it immobilizes the first few methylene groups in the tails of the lipid chains and therefore decreases the fluidity of the bilayer ${ }^{64}$, leading to stiffer membranes. 


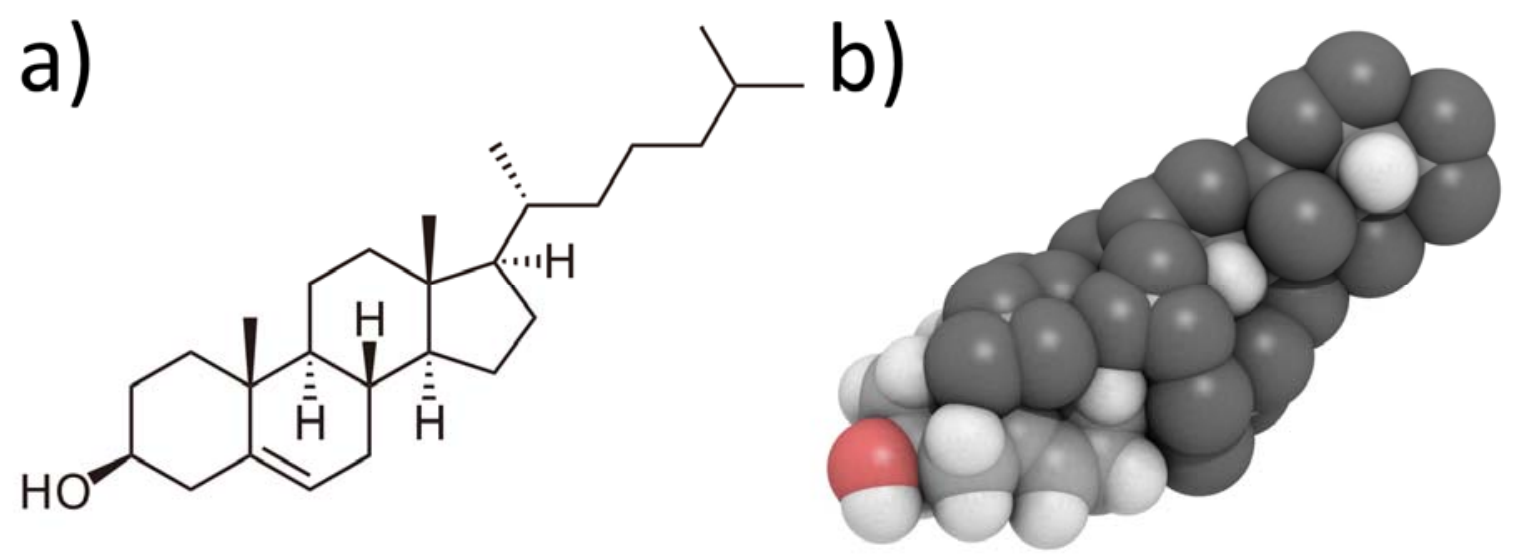

Figure 4.1 a) The skeletal and b) the space-filling molecular models of cholesterol showing the molecule's large planar ring structure attached to one polar hydroxyl group on one side and to a short nonpolar hydrocarbon chain on the other side.

\subsubsection{Biological Significance of Cholesterol}

Cholesterol content in membranes can vary considerably among different biological systems, reaching as high as 50 molar $\%$ of all the lipids in the membrane. ${ }^{63,64,152}$ Changes in cholesterol content was shown to be an indication of pathological disorders ranging from coronary heart disease ${ }^{153}$ to neurodegenerative diseases, ${ }^{154}$ in addition to many types of malignancies ${ }^{66}$ For instance, observations of anomalous cholesterol content in the plasma membrane have been reported in some types of cancers. ${ }^{155-158}$ This is generally the direct outcome of dysregulation in the feedback mechanisms by which the cholesterol content of the membrane is controlled. ${ }^{159}$ Changes in the cholesterol molar ratio of red blood cells' (RBC) membrane have been linked to changes in the deformability of those cells. Deformability-impaired RBCs are increasingly postulated to contribute to various types of anemias, ${ }^{160-164}$ cardiovascular conditions, ${ }^{165-170} \operatorname{liver}^{171}$ and renal ${ }^{172-}$ ${ }^{176}$ diseases, diabetes, ${ }^{177-183}$ and viral infections. ${ }^{184,185}$ Therefore, cellular stiffness can be used as a marker indicative of various pathological disorders. Table 4-1 tabulates some of the pathological conditions in which a correlation has been made between the changes in the fluidity of the RBCs' membranes and the changes in the cholesterol to phospholipid ratio of the membrane. 
Table 4-1 RBC membrane fluidity changes in different diseases and the associated changes in the cholesterol to phospholipid ratio (C/P).

\begin{tabular}{|c|c|c|c|c|c|}
\hline Disease & Change & Main Reason & $\mathrm{C} / \mathrm{P}$ ratio & Method & Ref \\
\hline Type I diabetes & Decrease & Lipid disorders & Increase & $\mathrm{FP}^{*}$ & {$[186]$} \\
\hline Diabetes & Decrease & $\begin{array}{c}\text { Alteration in membrane } \\
\text { phospholipid classes and their } \\
\text { acyl-chains }\end{array}$ & $\begin{array}{c}\text { Not } \\
\text { correlated }\end{array}$ & $\mathrm{ESR}^{* *}$ & [187] \\
\hline $\begin{array}{l}\text { Alcoholic liver } \\
\text { disease }\end{array}$ & Decrease & $\begin{array}{c}\text { Phosphatidylcholine/sphingomy } \\
\text { elin }(\mathrm{PC} / \mathrm{SM}) \text { increase and } \\
\text { unsaturated/saturated fatty acid } \\
\text { ratio decrease }\end{array}$ & N/A & $\begin{array}{l}\text { Fluorescence } \\
\text { recovery time }\end{array}$ & $188]$ \\
\hline Cervical cancer & Decrease & $\begin{array}{l}\text { Lipid peroxidation, insufficient } \\
\text { antioxidant potential }\end{array}$ & Increase & Chemical & 189] \\
\hline $\begin{array}{l}\text { Myotonic } \\
\text { muscular } \\
\text { dystrophy }\end{array}$ & Increase & $\begin{array}{l}\text { Maybe alterations in the } \\
\text { phosphorylated polypeptides of } \\
\text { membrane }\end{array}$ & N/A & ESR & {$[190]$} \\
\hline Psoriasis & Decrease & Lipid peroxidation & Increase & ESR & {$[191]$} \\
\hline Schizophrenia & Decrease & $\begin{array}{l}\text { Haloperidol treatment } \\
\text { withdrawal }\end{array}$ & Increase & $\begin{array}{c}\text { Fluorescent } \\
\text { probe }\end{array}$ & {$[192]$} \\
\hline $\begin{array}{l}\text { Magnesium } \\
\text { deficiency }\end{array}$ & Increase & $\begin{array}{c}\text { Decreased amounts of } \\
\text { magnesium, cholesterol, and } \\
\text { sphingomyelin }\end{array}$ & Decrease & FP & 193] \\
\hline
\end{tabular}

\subsection{Vesicles with Binary Compositions}

It was demonstrated earlier that, during heating the transition in a vesicle's acoustic contrast factor $(\phi)$ from positive nodal focusing to negative anti-nodal focusing occurs at a specific temperature dubbed the acoustic contrast temperature $\left(T_{\phi}\right)$. This $T_{\phi}$ is dependent on the compressibility and density of both vesicle and medium. For the vesicle, the membrane which is composed of phosphatidylcholines such as DMPC and DPPC undergoes a series of phase transitions with changing temperature. ${ }^{69}$ These thermotropic transitions impart a significant change in the mechanical properties of vesicles' membrane. ${ }^{91,125,126}$ For instance, as the membrane undergoes a transition from a gel to a ripple phase and then from the ripple to a liquid phase the compressibility of the vesicle increases. ${ }^{134}$ The first transition between two gel phases $\left(\mathrm{L}_{\beta^{\prime}} \rightarrow \mathrm{P}_{\beta^{\prime}}\right)$ 
is known as the pre-transition and it happens at the pre-transition temperature $\left(T_{p}\right)$. The second transition which is from the ripple gel phase to a fluid phase $\left(\mathrm{P}_{\beta^{\prime}} \rightarrow \mathrm{L}_{\alpha}\right)$ is known as the main transition and it occurs at the main transition temperature $\left(T_{m}\right)$. For POPC, however, the solid phase directly converts to the fluid phase upon heating in excess water conditions $\left(\mathrm{L}_{\beta} \rightarrow \mathrm{L}_{\alpha}\right)$. In the common two-letter nomenclature used to classify the lipid-water phases, an upper-case Latin letter is used to denote the long-range order and a lower-case Greek suffix is used to denote the shortrange order of the lipid chain. The upper-case letter $\mathrm{L}$ refers to one-dimensional (lamellar) and $\mathrm{P}$ refers to two-dimensional oblique or centered order. The lower-case letter $\alpha$ is a disordered (fluid) and $\beta$ is a partly ordered (elongated) type of conformation. The prime sign on the $\beta$ denotes that the chains are tilted. ${ }^{69}$

It was shown in Figure 3.9 that at the main transition from the ordered state to the disordered state the lateral crystalline arrangement and chain order of the lipid molecules are lost. These changes in the state of membrane bring about considerable changes in thermoelastic properties such as bending rigidity and compressibility. ${ }^{134}$

The selection of PCs for the preparation of the vesicles for cholesterol-containing vesicles was based on natural abundance and availability of thermotropic and stiffness data in the literature. The membrane compositions of some common cells containing these PCs are tabulated in Table 2-2. The data in the phase diagrams were obtained from the literature for various binary compositions of DMPC, ${ }^{194}$ DPPC, ${ }^{195}$ and POPC ${ }^{196}$ system with cholesterol, based on the average values obtained from differential scanning calorimetry, X-ray diffraction, NMR spectroscopies, spin-label ESR spectroscopy studies, and statistical thermodynamic calculations. The bending rigidity data were obtained from the literature for $\mathrm{DMPC},{ }^{86} \mathrm{DPPC},{ }^{197}$ and $\mathrm{POPC}^{198}$ at various cholesterol contents. 


\subsubsection{Thermo-Acoustophoretic Behavior of DMPC-Cholesterol System}

DMPC vesicles with various contents of cholesterol, up to $X_{\text {chol }}=0.3$ were synthesized to investigate the effect of effect of $X_{\mathrm{chol}}$ on $T_{\phi}$. In a typical experiment, the vesicle suspension was injected into the microfluidic channel while the signal generator was set to the first harmonic frequency determined prior to the experiments using initial conditions. A prompt focusing response of vesicles to the nodal region was typically observed. A controllable temperature increase was then initiated while the vesicles were visually monitored under a fluorescence microscope. The temperature at which the vesicles initiated their migration towards the anti-nodal regions (the walls in a first harmonic channel) was recorded as the $T_{\phi}$ of that particular vesicles composition. The values of $T_{\phi}$ were evaluated visually by observing the behavior of the vesicles. Using a captured video, $T_{\phi}$ was the temperature at which the vesicles initiated their anti-nodal migration. Ideally, an image processing approach can be used to determine $T_{\phi}$ from the fluorescent intensity changes using an established threshold; however, at this moment it remains user-determined. In all systems, only a single point is identified as the $T_{\phi}$ during the heating experiment. However, temperatures recorded as the $T_{\phi}$ on heating differed slightly with the ones observed during cooling. This difference, was less than $2{ }^{\circ} \mathrm{C}$ (at heating/cooling rates of $5{ }^{\circ} \mathrm{C} \min ^{-1}$ ) and was further minimized by slowing down the temperature cooling sweep to less than $0.5^{\circ} \mathrm{C}$ (at heating/cooling rates of 0.5 $\left.{ }^{\circ} \mathrm{C} \min ^{-1}\right)$. The temperature sweep was coupled with a manual frequency sweep to adjust for medium's property changes and maintain first harmonic frequency conditions. The $T_{\phi}$ values recorded in Figure 4.2 are averages of at least five separate vesicle batches. The trend of the change of $T_{\phi}$ with $X_{\text {chol }}$ was consistent for various PCs as can be observed in Figure 2a-c. This trend was also consistent with the $k_{c}$ vs $X_{\text {chol }}$ trend reported elsewhere. ${ }^{86}$ It is evident from the plot that the addition of cholesterol to up to $X_{\text {chol }}=0.3$ in DMPC vesicles significantly increased the $k_{c}$. 


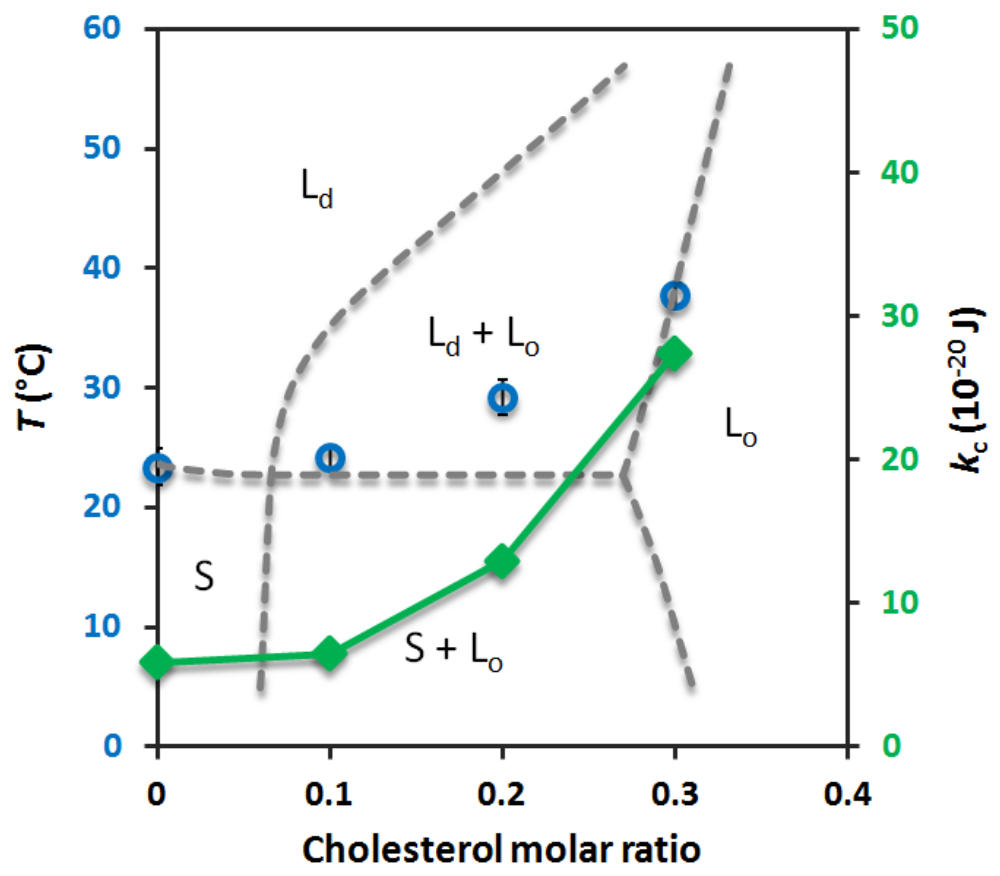

Figure 4.2 Determination of the acoustic contrast temperature, $T_{\phi}$, (circles) for the DMPC:Chol binary mixtures. The background dashed lines represent the binary equilibrium phase diagram of the system, plotted using the data available in the literature. ${ }^{194} \mathbf{S}$ denotes solid, $\mathrm{L}_{\mathbf{o}}$ liquid ordered, and $\mathrm{L}_{\mathbf{d}}$ liquid disordered phases. Diamond markers represent the $\boldsymbol{k}_{c}$ at specific $X_{\text {chol }}$ at room temperature obtained from X-ray scattering methods. ${ }^{86}$

\subsubsection{Thermo-Acoustophoretic Behavior of DPPC-Cholesterol System}

Figure 4.3 shows the binary phase diagram of the DPPC:Chol system superimposed with the changes of $k_{c}$ vs $X_{\text {chol }}$ for the same system. As is evident from the figure, the changes of $k_{c} v s$. $X_{\text {chol }}$ does not exhibit a similar trend as what was observed for the DMPC:Chol system. This is not surprising as the effect of cholesterol on the membrane's mechanical properties has been shown to be specific to the lipid type. ${ }^{86,199}$ In the case of DPPC, the $k_{c}$ of the membrane first decreases at low cholesterol contents, which is followed by an increase at around 25 molar $\%$ of cholesterol, ending with a relative decrease at 40 molar $\%$ of cholesterol. The decrease in the stiffness at low cholesterol concentrations is likely due to the fluidization of the membrane at those concentrations, ${ }^{200}$ since cholesterol has been shown to disrupt the long-range lateral order of the membrane. ${ }^{200}$ In this 
regard, vesicles with various contents of cholesterol, up to $X_{\text {chol }}=0.4$ were synthesized to investigate the effect of $X_{\text {chol }}$ on $T_{\phi}$ and examine the consistency of the $T_{\phi}$ with the changes of the $k_{c}$. Analogous to the DMPC:Chol system, a prompt focusing response of vesicles to the nodal region was typically observed. After controllable temperature increase was then initiated while the vesicles were visually monitored under the microscope. Similarly, for all compositions, only a single point is identified as the $T_{\phi}$ during the heating experiment, with a slight difference in the heating and cooling $T_{\phi}$ which was minimized by slowing down the heating/cooling rate. The $T_{\phi}$ values, which were the averages of at least five separate vesicle batches, are shown in Figure 4.3. As can be observed in the figure the trend of the change of $T_{\phi}$ with $X_{\text {chol }}$ is again consistent with the $k_{c}$ vs $X_{\text {chol }}$ trend reported elsewhere. ${ }^{197}$ The effect of cholesterol on the $T_{\phi}$ is however less apparent for DPPC for up to $X_{\text {chol }}=0.4$ compared to DMPC.

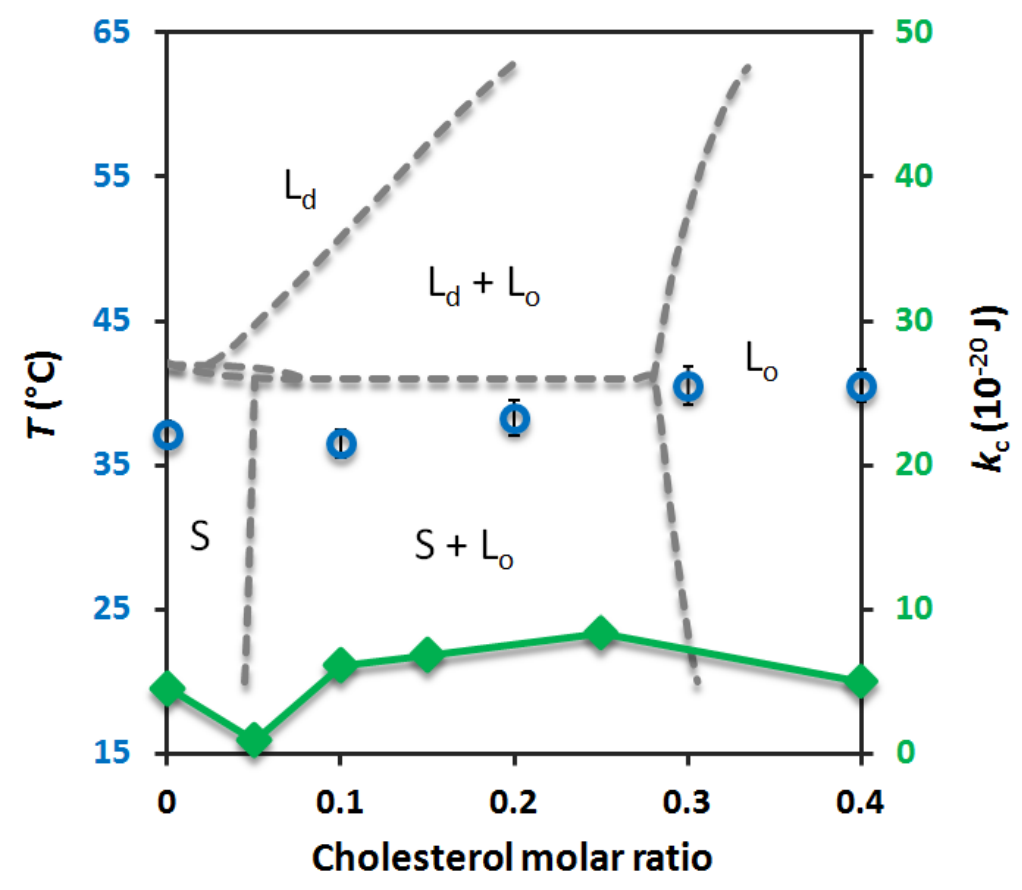

Figure 4.3 Determination of the acoustic contrast temperature, $T_{\phi}$, (circles) for the DPPC:Chol system. The background dashed lines represent the equilibrium phase diagram of the system, plotted using the data from the literature. ${ }^{195}$ Diamond markers represent the $k_{c}$, at room temperature obtained from molecular dynamic simulations. ${ }^{197}$ 


\subsubsection{Thermo-Acoustophoretic Behavior of POPC-Cholesterol System}

The same experiment was carried out for the POPC:Chol system (Figure 4.4). For POPC with one mono-unsaturated lipid chain, cholesterol has a rigidifying effect on the membrane, but not as pronounced as its effect on DMPC. The effect of cholesterol on the $T_{\phi}$ is however consistent with the changes of the stiffness with cholesterol content. The $T_{\phi}$, in all cases, does not exactly correspond to a distinct phase transition, rather a specific point at which the compressibility of the vesicles reaches a value that would make the $\phi$ negative. In other words, the thermotropic changes in stiffness which render the vesicles as roughly as compressible as water do not necessarily lead to a negative $\phi$ at any point close to the actual temperature of the phase transitions. For instance, POPC:Chol system, in which the main thermotropic transition occurs at a subzero temperature, as can be seen in Figure 4.4, but its reducing effect on the $k_{c}$ becomes evident at a higher temperature.

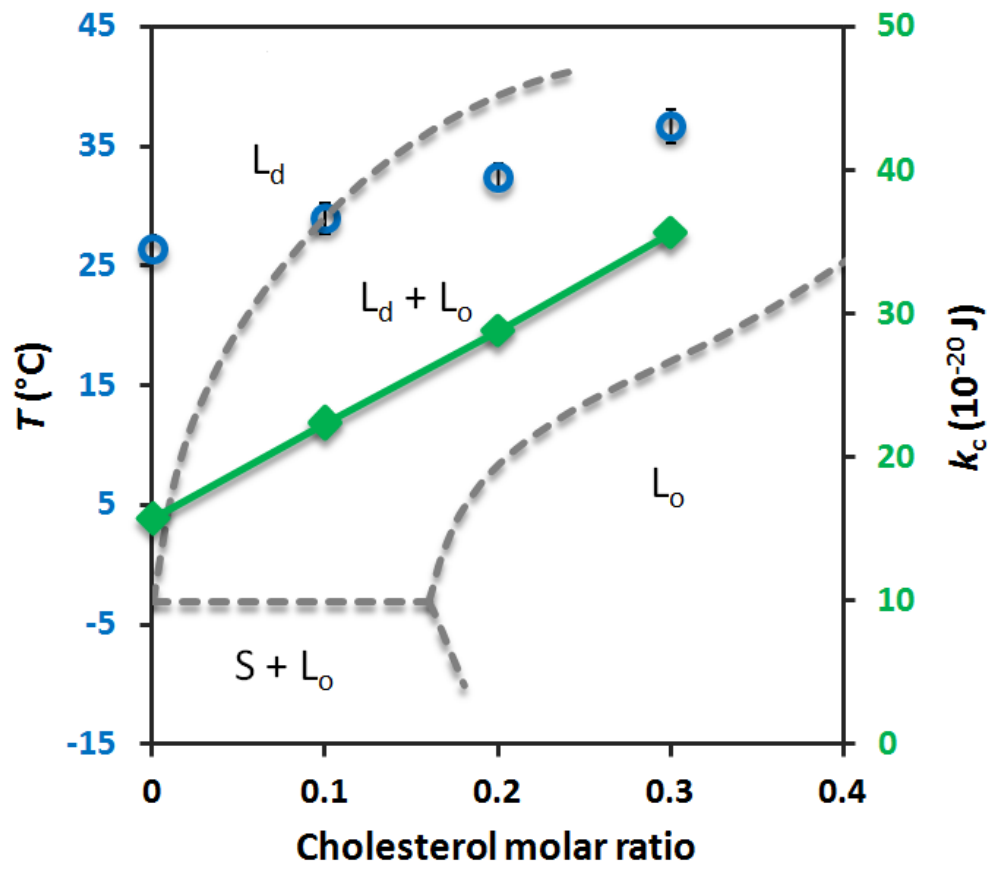

Figure 4.4 Determination of the acoustic contrast temperature, $T_{\phi}$, (circles) for the binary POPC:Chol system. The background dashed lines represent the binary equilibrium phase diagram of the system, plotted using the data available in the literature. ${ }^{196}$ Diamond markers represent the bending stiffness modulus, $\boldsymbol{k}_{\boldsymbol{c}}$, at specific $\boldsymbol{X}_{\mathrm{chol}}$ at room temperature obtained from vesicle fluctuation analysis studies. ${ }^{198}$ 


\subsection{Thermo-Acoustophoretic Behavior of Vesicles with Ternary Compositions}

Membranes composed of more than two PCs are more commonly abundant in nature. In such systems, the $X_{\text {chol }}$ may have competing effects on the membrane stiffness, depending on the type of PCs it contains. In order to investigate the effect of cholesterol on a DMPC:DPPC system's $T_{\phi}$, an experiment of 45 individual compositions of DMPC:DPPC:Chol vesicles were prepared. Using the individual $T_{\phi}$ values for each composition measured using the microfluidic device; the contour plot shown in Fig. 3 was developed. Analogous to the binary mixtures, a single point is identified in all cases as the acoustic contrast temperature of the vesicles in a heating experiment. Furthermore, there exists a difference between the temperature registered as the $T_{\phi}$ on heating and the one observed on cooling, which does not exceed $2{ }^{\circ} \mathrm{C}$ and could be minimized by slowing down the temperature sweep. From Fig. 3 one can learn that along the DMPC:DPPC binary axis, the measured $T_{\phi}$ slopes gradually increase from the minimum point of $23.2^{\circ} \mathrm{C}$ for the pure DMPC up to $37.6^{\circ} \mathrm{C}$ for the pure DPPC. This is, in fact, observed as the overall trend of the diagram especially at the base of the triangle where cholesterol content does not exceed 20\%. For the binary DMPCcholesterol system, $T_{\phi}$ shows a sharp increase from the minimum point of $23.2^{\circ} \mathrm{C}$ for pure DMPC up to $37.8{ }^{\circ} \mathrm{C}$ for the system with $30 \%$ cholesterol. On adding DPPC to the binary mixtures, the same trend but with less intensity is observed for the ternary compositions for up to around $60 \%$ of DPPC. For ternary compositions with more than $60 \%$ DPPC, the changes in $T_{\phi}$ is consistent with the decreasing trend observed for the binary DPPC:Chol system, in which $T_{\Phi}$ first decreases upon addition of cholesterol and then slightly increases up to the maximum value of $40.5{ }^{\circ} \mathrm{C}$ for pure DPPC. Finally, it is interesting to mention that even in vesicles with more complex membranes, despite the normally nonuniform mixing of the membrane components, a single $T$ can be identified as the $T_{\phi}$. This mapping of the $T_{\phi}$ allows us to study of the feasibility of a separation system that can enable the design of the separation of ternary vesicles with various compositions.. 


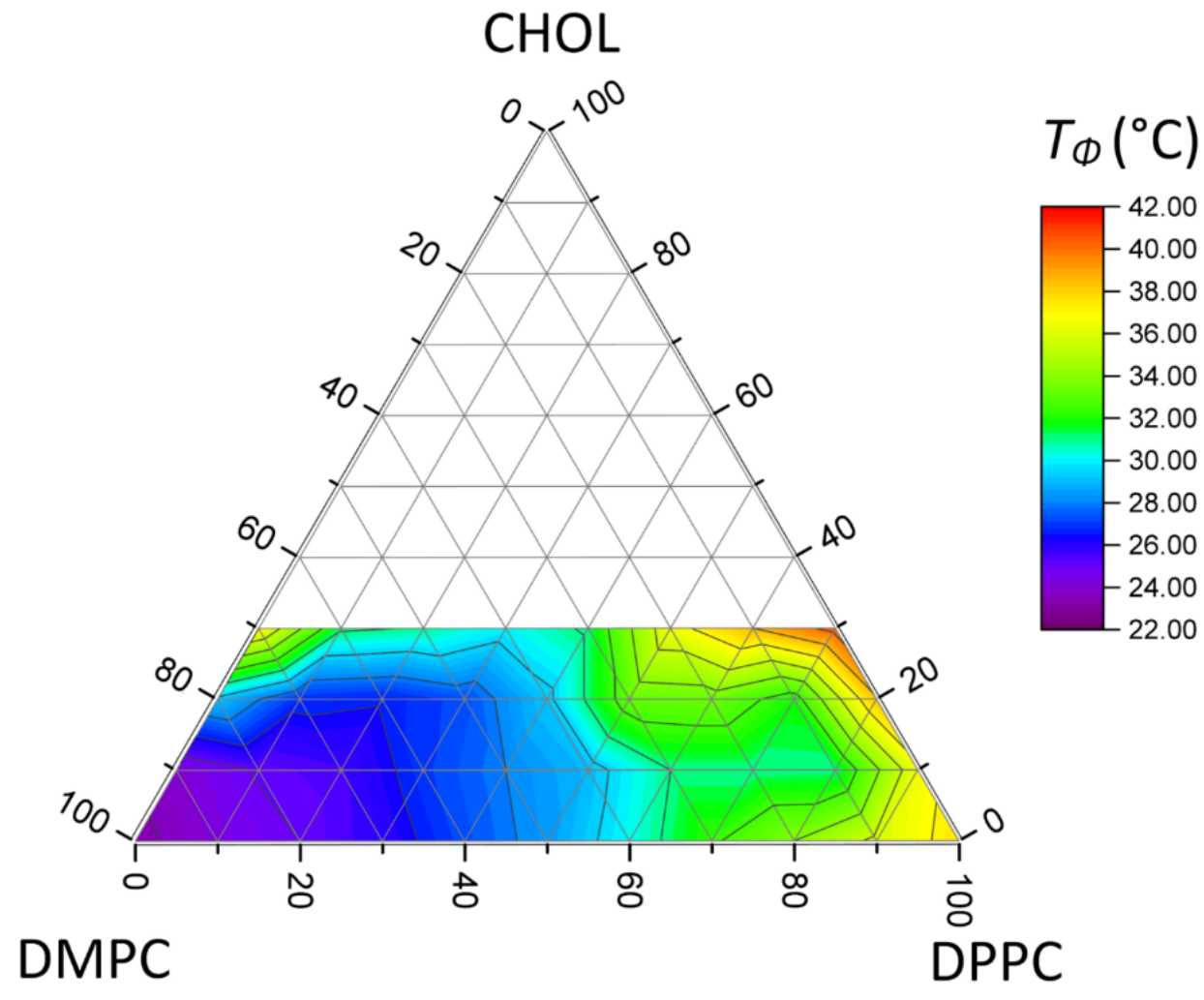

Figure 4.5 Triangular contour map of the measured $T_{\phi}$ for the DMPC:DPPC:Chol system up to the $X_{\text {chol }}=0.3$. The coloured surface indicating the value of $T_{\phi}$ was obtained using the data of 45 different points across the region of interest by thermo-acoustophoretic heating experiments. Of these 45 points, 38 of them were the nodes of the black triangles. The remining 7 were additional mid-node points that were also obtained to confirm the behavior in the 50:30:20 DMPC:DPPC:Chol region and to get higher resolution on the $T_{\phi}$ behavior. Black lines that appear at every fifth contour line mark a temperature difference of $1.5^{\circ} \mathrm{C}$. The overall trend in the diagram exhibits an increase in the $T_{\phi}$ from the minimum in the region shaded purple to the maximum in either the binary 70:30 DMPC:Chol or 70:30 DPPC:Chol systems. The $T_{\phi}$ values shown here are averages of at least three separate experiments.

\subsection{Demonstration of Thermo-Acoustophoresis}

Since vesicle systems of different $X_{\text {chol }}$ were shown to exhibit different $T_{\phi}$ values, the thermo-acoustofluidic separation of such vesicles could be developed. In order to determine the temperature at which the acoustophoresis can occur, the calculated $\phi$ of each of the vesicles in pure and incorporated with 30 molar\% of cholesterol was plotted against temperature in Figure 4.6a and b. These values were calculated using the compressibility and sound velocity measurements data of pure PC and PC:Chol systems available in the literature. ${ }^{201,202}$ Appendix IV presents the 
derivation of $\phi$ using the sound velocity measurements and compressibility data. In Figure 4.6a and $\mathrm{b}$, the intercept with the abscissa in each vesicle system denote the temperature at which $\phi=$ 0 , also defined as the $T_{\phi}$. In Figure 4.6a the range of temperatures between the $T_{\phi}$ of $X_{\text {chol }}=0$ and the $T_{\phi}$ of $X_{\text {chol }}=0.3$ can be considered as the separation "window" for the acoustophoresis. This means that a separation temperature set within this window would cause the migration of vesicles in opposite directions (i.e. wall and center), thus yielding a separation ( $T_{\phi, 100: 0 \text { PC:Chol }}<$ $T_{\text {sepration }}<T_{\phi, 70: 30 \text { PC:Chol }}$. The window was $13.4{ }^{\circ} \mathrm{C}$ wide for DMPC:Chol and $3.9{ }^{\circ} \mathrm{C}$ wide for DPPC:Chol systems (Figure 4.6b). These values corroborate the experimentally observed difference in $T_{\phi}$ in the DMPC:Chol and DPPC:Chol systems. The widths of the windows were significantly different considering that both systems were composed for $X_{\text {chol }}=0$ and $X_{\text {chol }}=0.3$. However, the effect of cholesterol content on each of the PCs yielded a different degree of stiffness change as discussed earlier.
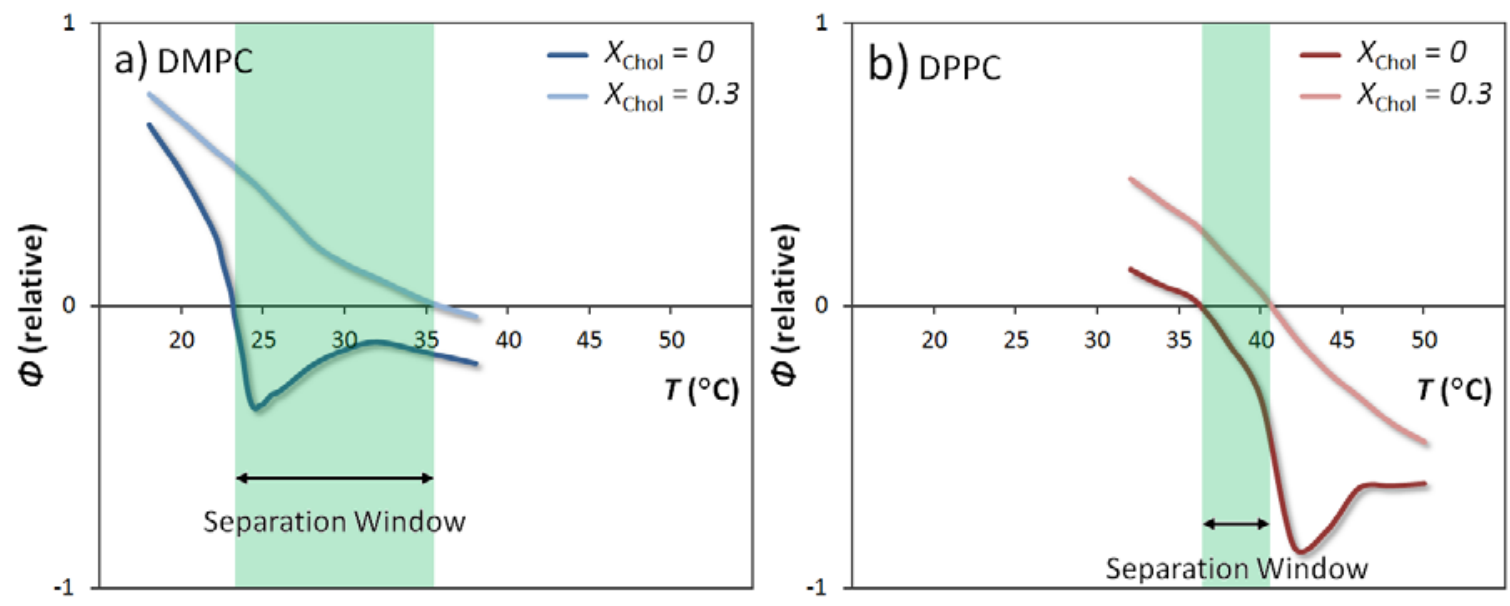

Figure 4.6 Determination of the separation window for DMPC:Chol and DPPC:Chol systems by calculating the relative values of $\phi$ for a) DMPC and b) DPPC vesicles in pure state and in $X_{\text {chol }}=0.3$. The temperature windows are marked in green and are considered the temperature range within which separation could be achieved due to opposite migration direction of the vesicles $\left(T_{\phi, 100: 0}\right.$ PC:Chol $<$ $T_{\text {separation }}<T_{\phi, 70: 30 \mathrm{PC}: \text { Chol }}$ ). The relative values of $\phi$ were calculated using vesicle sound velocity measurements and compressibility data from the literature. ${ }^{201,202}$ 
To examine the feasibility of the separation of more than two vesicle systems via thermoacoustophoresis, vesicles with distinct membrane compositions were prepared and examined in a multi-stage device maintaining two distinct temperatures of $27^{\circ} \mathrm{C}$ and $31^{\circ} \mathrm{C}$ at its two stages using mini-Peltier elements (Figure 4.7c). The selected compositions were $X_{\text {chol }}=0.1,0.2$, and 0.3 , which were fluorescently tagged with green, red, and blue, respectively. In all cases, the total lipid used in the preparation of the vesicles was $2 \mathrm{wt} \%$, yielding vesicles with an average diameter of $11.75 \pm 0.71 \mu \mathrm{m}$. The $T_{\phi}$ for these compositions was pre-measured as $24.2^{\circ} \mathrm{C}$ for $X_{\text {chol }}=0.1,29.2$ ${ }^{\circ} \mathrm{C}$ for $X_{\text {chol }}=0.2$, and $37.8^{\circ} \mathrm{C}$ for $X_{\text {chol }}=0.3$. Since the layout of the channel schematically shown in Figure 4.7 was modified to accommodate multi-stage separations, the new chip employed two Peltier elements under the main channel preceding the splits. The second actuator was used when necessary to accommodate for the temperature-induced changes in the first harmonic frequency. In a typical separation, when both Peltier elements and actuators are switched off $\left(T=23{ }^{\circ} \mathrm{C}\right)$, all vesicles were randomly dispersed (Figure 4.7a).

Upon actuation $\left(f=1.33 \mathrm{MHz}, 50 \mathrm{~V}_{p p}\right)$ at the same temperature, all vesicles swiftly migrated toward the central region of the channel, i.e. the node (Figure 4.7b). As the separation temperature was below all $T_{\phi}$ values for the various compositions, the nodal migration was expected. After the first Peltier element was switched on and the first stage channel reached a temperature of $\sim 27{ }^{\circ} \mathrm{C}$, the separation temperature exceeded the $T_{\phi}$ of the $X_{\text {chol }}=0.1$ while remained lower than the $T_{\phi}$ of the $X_{\text {chol }}=0.2$ and 0.3 . Under these conditions, $X_{\text {chol }}=0.2$ and 0.3 vesicles remained well-focused at the node in the center of the channel, while $X_{\text {chol }}=0.1$ vesicles migrated towards the walls (Figure 4.7d). These vesicles were gradually collected from the first outlet while the remaining vesicles proceeded to the second stage of the separation. At the second stage of the separation the Peltier element was set to a setting to achieve an average second stage channel temperature of $\sim 31{ }^{\circ} \mathrm{C}$ at the given conditions. Under these conditions the separation 
temperature exceeded the $T_{\phi}$ of the $X_{\text {chol }}=0.2$ while remaining lower than the $T_{\phi}$ of the $X_{\text {chol }}=$ 0.3 ; therefore, the $X_{\text {chol }}=0.2$ vesicles migrated towards to the walls while the $X_{\text {chol }}=0.3$ vesicles remained focused at the center (Figure 4.7e). The wall focused vesicles in the second stage were collected from outlet 2, while the second stage main channel vesicles were collected from outlet 3 .

The efficiency of the separation was dependent on many parameters out of which only the temperatures of the first and second stages of the separation was varied while the rest were maintained constant. The vesicles in each of the injected samples included equal counts of each of the three compositions of vesicles to minimize concentration-caused disturbances. Mixtures were also mixed immediately before the separation experiments to minimize the exchange of cholesterol between the various $X_{\text {chol }}$ vesicles. The flow rate was also controlled and remained constant during all experiments at a rate of $5 \mu 1 \mathrm{~min}^{-1}$. In order to study the effect of temperatures on the separation efficiency, four different experiments were designed using identical vesicles mixtures of $X_{\text {chol }}=$ $0.1,0.2$, and 0.3 in equal counts pre-labeled with green, red, and blue fluorescent tags, respectively. The temperatures of the two stages of the separation were considered $T_{1}$ for stage one and $T_{2}$ for stage two and were controlled by two Peltier elements while holding all other parameters virtually constant. The efficiency of the separation was assessed by measuring the percentage of $X_{\text {chol }}=0.1$ vesicles in outlet $1, X_{\text {chol }}=0.2$ vesicles in outlet 2 , and $X_{\text {chol }}=0.3$ vesicles in outlet 3 using fluorescence signals. These values are summarized in the bar graph in Figure 4.8. When both stages were set to $23{ }^{\circ} \mathrm{C}$ (room temperature), all three vesicles were nodally focused and no vesicles were collected in outlets 1 and 2 . This caused all three types of vesicles to exit from outlet 3 , leading to an outlet 3 purity of $33.9 \%$. When both stages' temperatures where increased to $25^{\circ} \mathrm{C}$, the first stage separation operated efficiently since the separation temperature exceeded the $T_{\phi}$ of $X_{\text {chol }}=$ 0.1 vesicles and thus a purity in outlet 1 of $95.4 \%$ was achieved. The second stage separation did not operate efficiently however and both vesicles were nodally focused and exited from outlet 3 causing outlet 3 purity to be $49.0 \%$. When both stages were raised to $27^{\circ} \mathrm{C}$ the same behavior was 
observed since the second stage temperature did not exceed the $T_{\phi}$ of the $X_{\text {chol }}=0.2$ vesicles of $29.2{ }^{\circ} \mathrm{C}$. However, when the second stage temperature was increased to $31^{\circ} \mathrm{C}$ both stages of the separation operated efficiently and purities reached $94.2 \%, 96.5 \%$, and $93.1 \%$ for outlets 1,2 , and 3 , respectively. The conditions of these two stages are identical to the conditions shown in Figure 4.7d-e. These results confirm that thermo-acoustophoresis is a temperature-enabled separation that unless properly designed, simple acoustophoresis would not yield a separation.
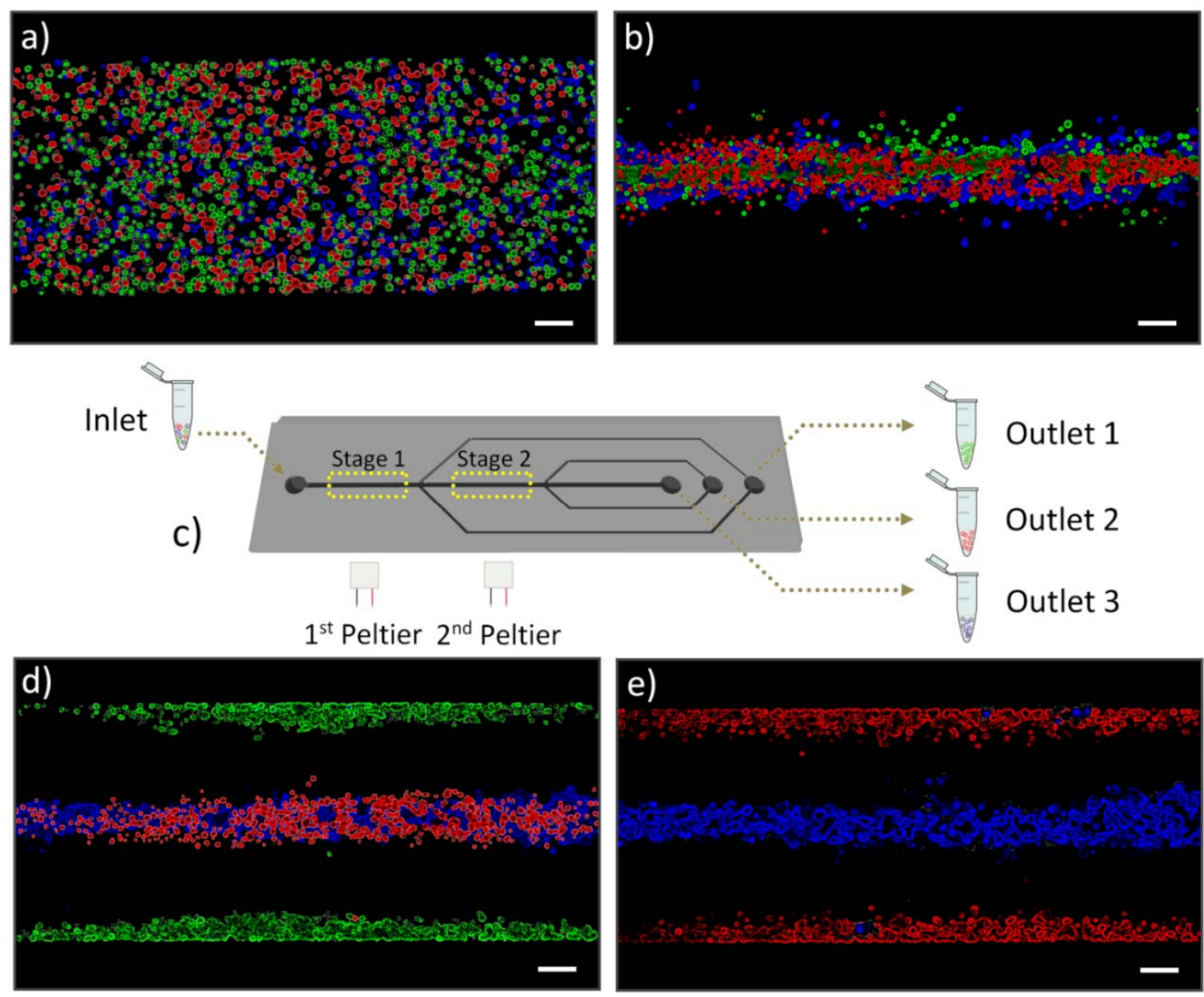

Outlet 1

Outlet 2

Outlet 3

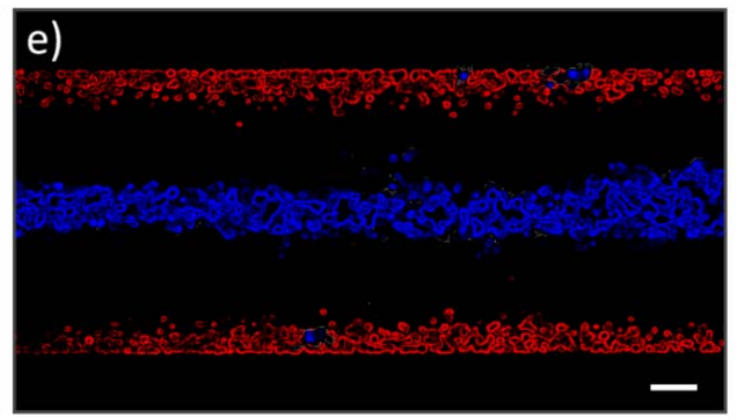

Figure 4.7 Optical fluorescence micrographs of various stages of the multi-stage thermoacoustophoretic separation with (a) channel view with Peltier elements and actuators switched off showing randomly dispersed vesicles of $X_{\text {chol }}=0.1$ (green), $X_{\text {chol }}=0.2$ (red), and $X_{\text {chol }}=0.3$ (blue) at $T=23{ }^{\circ} \mathrm{C}$, (b) actuator switched on to first harmonic frequency with Peltier elements remaining off showing all vesicles focusing to the nodal region, (c) schematic of the multi-stage separation channel showing the two stages of separation and the inlet/outlets, (d) first stage channel temperature set to 27 ${ }^{\circ} \mathrm{C}$ leading to the migration of the $X_{\text {chol }}=0.1$ vesicles to the anti-nodal zones, (e) second stage temperature set to $31{ }^{\circ} \mathrm{C}$ leading to the migration of the $X_{\text {chol }}=0.2$ vesicles to the anti-nodal zones. (Scale bars in all images are $80 \mu \mathrm{m}$ ). 


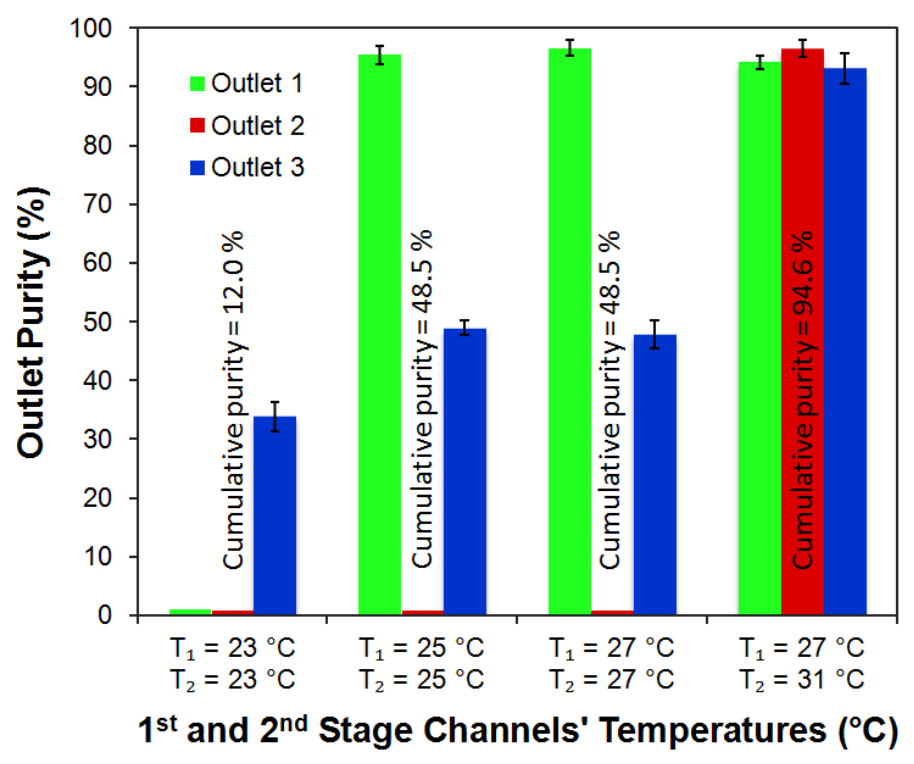

Figure 4.8 Purity of collected vesicles samples from outlets 1, 2, and 3 at various temperature conditions of the two stages of the separation. Purities were quantified based on the intended vesicle for each outlet with $X_{\text {chol }}=0.1$ in outlet $1, X_{\text {chol }}=0.2$ in outlet 2 , and $X_{\text {chol }}=0.3$ in outlet 3 . The cumulative purities were calculated for each temperature condition scenario using the purity of outlets 1,2 , and 3.

\subsection{Conclusion}

In conclusion, thermo-acoustophoretic behavior of different binary mixtures of three phosphatidylcholines (DMPC, DPPC, and POPC) with cholesterol was evaluated using a firstharmonic channel operated in heating and cooling modes. In all cases, the mixtures exhibited a single acoustic contrast temperature, which corresponded to the point at which that mixture became roughly as compressible as water. The increase in $T_{\Phi}$ for cholesterol-containing vesicles showed a similar trend with the ordering phenomenon in vesicles, and the accompanying changes in stiffness. Furthermore, thermo-acoustophoresis was used in this study to enable the separation of a threevesicle system with variable $X_{\text {chol }}$ values. By taking advantage of the composition-dependent stiffness, which endowed vesicles with unique $T_{\phi} \mathrm{s}$, vesicles were separated in a 2-stage separation device. The separation of vesicles was achieved by operating in the temperature range between the vesicles' $T_{\phi}$ values considered as the separation "window". The separation was demonstrated in a 
multi-stage device for a three-vesicle system with $X_{\text {chol }}$ ranging from 0.1 to 0.3 . The thermoacoustofluidic device had separation efficiencies exceeding $93 \%$ for the three-vesicle system. We believe this is the first report on the separation of vesicles based on $X_{\text {chol }}$. We conclude that thermoacoustophoresis may be well suited for applications in bioanalytical sciences, especially for the isolation of cells (e.g. erythrocytes) and extracellular vesicles (e.g. exosomes and microvesicles). 


\section{Summary}

This dissertation presents a new method for the composition-based separation of membrane-bound biological systems such as vesicles using a thermally-assisted ultrasonicallyactuated microfluidic platform. The focusing behavior of vesicles enclosed by bilayers constituting of unary, binary, and ternary lipid compositions were examined in an ultrasonic standing wave designed in the first harmonic mode. Investigation into the behavior of bilayers upon changing the temperature revealed a considerable change in the mechanical properties of the membrane, expressed through a switch in the focusing of the vesicles from the node to antinodes of the ultrasonic wave and vice versa. The specific conclusions on the behavior of vesicles in the thermally-assisted acoustofluidic device and the potential of this method to be used in bioanalytical applications are listed below:

- We have demonstrated that membrane-enclosed systems such as vesicles would typically exhibit a positive $\phi$ and therefore they can be nodally focused in an ultrasonic standing wave.

- Reversible thermotropic changes in the lipid bilayer of vesicles rendered the vesicles compressible or stiff enough to undergo a $\phi$ sign change and reposition into the antinodes or nodes of the standing wave, respectively.

- This point at which the acoustic contrast factor of the vesicles became zero was dubbed the acoustic contrast factor transition temperature or for brevity acoustic contrast temperature $\left(T_{\phi}\right)$ and was found to be specific to the composition of the vesicle.

- The observation of the changes in the $\phi$ sign was corroborated using compressibility data to calculate the $T_{\phi}$ values, which closely matched the $T_{\phi}$ measurements obtained in this study.

- This technique allowed us to exploit the unique acoustic contrast temperatures of vesicles to yield a separation at $T_{\phi 1}<T_{\text {separation }}<T_{\phi 2}$. Under this separation temperature condition, 
vesicles migrated in opposite directions, towards the central region or the wall regions of the channel.

- Thermo-acoustophoresis was successfully employed to enable the separation of a three-vesicle system with variable $X_{\text {chol }}$ values. By taking advantage of the composition-dependent stiffness, a unique $T_{\phi}$ values for each vesicle system were obtained. The separation was demonstrated in a multi-stage device for a three-vesicle system with $X_{\text {chol }}$ ranging from 0.1 to 0.3 .

- High separation efficiencies (over 95\%) were achieved and we anticipate that it can be further improved in the future by optimizing variables such as the volumetric flow rate, separation temperature, and the amplitude of the acoustic radiation.

- We envision that this method may be well suited as a stand-alone or in conjunction with other separation methods that can yield medical separations and diagnostics, especially as a tool for sorting cells and extracellular vesicles based on their origin cell types in complex biological fluid samples.

- This method could also be used as a reliable tool for analytical applications in liposomal research, drug-delivery liposomes, and separating liposomes or other membrane-enclosed systems based on capsulation. 


\section{Bibliography}

1. Whitesides, G. M. The origins and the future of microfluidics. Nature 442, 368-373 (2006).

2. Encyclopedia of Microfluidics and Nanofluidics. (Springer US, 2008). doi:10.1007/978-0387-48998-8

3. Microfluidics for Biological Applications. (Springer US, 2009). doi:10.1007/978-0-38709480-9

4. Sackmann, E. K., Fulton, A. L. \& Beebe, D. J. The present and future role of microfluidics in biomedical research. Nature 507, 181-189 (2014).

5. Alexandre Joel Chorin. Numerical solution of the Navier-Stokes equations. Math. Comput. 22, 745-762 (1968).

6. L. Quartapelle. Numerical Solution of the Incompressible Navier-Stokes Equations. (Birkhäuser Basel, 1993). doi:10.1007/978-3-0348-8579-9

7. Koo, J. \& Kleinstreuer, C. Liquid ow in microchannels: experimental observations and computational analyses of micro uidics effects. J. Micromechanics Microengineering 13, $568-579$ (2003).

8. Sommerfeld, A. Ein Beitrag zur hydrodynamischen Erkläerung der turbulenten Flüssigkeitsbewegüngen. in 4th International Congress of Mathematicians Vol. 3, pp. 116124 (1908).

9. Stone, H. A. \& Kim, S. Microfluidics: Basic issues, applications, and challenges. AIChE J. 47, 1250-1254 (2001).

10. G. K. Batchelor. An Introduction to Fluid Dynamics. (Cambridge University Press, 2002).

11. Bruus, H. Acoustofluidics 7: The acoustic radiation force on small particles. Lab Chip 12, 1014-21 (2012).

12. Wyatt Shields IV, C., Reyes, C. D. \& Lopez, G. P. Microfluidic cell sorting: a review of the advances in the separation of cells from debulking to rare cell isolation. Lab Chip 15, 1230 1249 (2015).

13. Huh, D., Gu, W., Kamotani, Y., Grotberg, J. B. \& Takayama, S. Microfluidics for flow cytometric analysis of cells and particles. Physiol. Meas. 26, R73-R98 (2005).

14. Holmes, D., Morgan, H. \& Green, N. G. High throughput particle analysis: Combining dielectrophoretic particle focussing with confocal optical detection. Biosens. Bioelectron. 21, 1621-1630 (2006).

15. Wilding, P., Pfahler, J., Bau, H. H., Zemel, J. N. \& Kricka, L. J. Manipulation and flow of biological fluids in straight channels micromachined in silicon. Clin. Chem. 40, 43 LP-47 (1994).

16. Murthy, S. K., Sethu, P., Vunjak-Novakovic, G., Toner, M. \& Radisic, M. Size-based microfluidic enrichment of neonatal rat cardiac cell populations. Biomed. Microdevices $\mathbf{8}$, 231-237 (2006). 
17. Voldman, J. Electrical forces for microscale cell manipulation. Annu. Rev. Biomed. Eng. 8, 425-454 (2006).

18. Herbert A. Pohl. Dielectrophoresis: the behavior of neutral matter in nonuniform electric fields. (Cambridge University Press, 1978).

19. Urdaneta, M. \& Smela, E. Parasitic trap cancellation using multiple frequency dielectrophoresis, demonstrated by loading cells into cages. Lab Chip 8, 550-556 (2008).

20. Lapizco-Encinas, B. H., Simmons, B. A., Cummings, E. B. \& Fintschenko, Y. Dielectrophoretic Concentration and Separation of Live and Dead Bacteria in an Array of Insulators. Anal. Chem. 76, 1571-1579 (2004).

21. Wang, X.-B. et al. Cell Separation by Dielectrophoretic Field-flow-fractionation. Anal. Chem. 72, 832-839 (2000).

22. Vykoukal, J., Vykoukal, D. M., Freyberg, S., Alt, E. U. \& Gascoyne, P. R. C. Enrichment of putative stem cells from adipose tissue using dielectrophoretic field-flow fractionation. Lab Chip 8, 1386-1393 (2008).

23. Md Ali, M. A., Ostrikov, K. (Ken), Khalid, F. A., Majlis, B. Y. \& Kayani, A. A. Active bioparticle manipulation in microfluidic systems. RSC Adv. 6, 113066-113094 (2016).

24. Forbes, T. P. \& Forry, S. P. Microfluidic magnetophoretic separations of immunomagnetically labeled rare mammalian cells. Lab Chip 12, 1471-1479 (2012).

25. Furdui, V. I. \& Harrison, D. J. Immunomagnetic T cell capture from blood for PCR analysis using microfluidic systems. Lab Chip 4, 614-618 (2004).

26. Pamme, N. \& Manz, A. On-Chip Free-Flow Magnetophoresis: Continuous Flow Separation of Magnetic Particles and Agglomerates. Anal. Chem. 76, 7250-7256 (2004).

27. Lenshof, A., Magnusson, C. \& Laurell, T. Acoustofluidics 8: Applications of acoustophoresis in continuous flow microsystems. Lab Chip 12, 1210 (2012).

28. Leibacher, I., Reichert, P. \& Dual, J. Microfluidic droplet handling by bulk acoustic wave (BAW) acoustophoresis. Lab Chip 15, 2896-2905 (2015).

29. Ashkin, A. Acceleration and Trapping of Particles by Radiation Pressure. Phys. Rev. Lett. 24, 156-159 (1970).

30. Ashkin, A., Dziedzic, J. M., Bjorkholm, J. E. \& Chu, S. Observation of a single-beam gradient force optical trap for dielectric particles. Opt. Lett. 11, 288-290 (1986).

31. Chiou, P. Y., Ohta, A. T. \& Wu, M. C. Massively parallel manipulation of single cells and microparticles using optical images. Nature 436, 370-372 (2005).

32. Lee, K. S. et al. Optofluidic particle manipulation in a liquid-core/liquid-cladding waveguide. Opt. Express 20, 17348-17358 (2012).

33. Huang, L. R., Cox, E. C., Austin, R. H. \& Sturm, J. C. Continuous Particle Separation Through Deterministic Lateral Displacement. Science (80-. ). 304, 987 LP-990 (2004).

34. Shevkoplyas, S. S., Yoshida, T., Munn, L. L. \& Bitensky, M. W. Biomimetic Autoseparation of Leukocytes from Whole Blood in a Microfluidic Device. Anal. Chem. 77, 933-937 (2005). 
35. Yamada, M., Nakashima, M. \& Seki, M. Pinched Flow Fractionation: Continuous Size Separation of Particles Utilizing a Laminar Flow Profile in a Pinched Microchannel. Anal. Chem. 76, 5465-5471 (2004).

36. Takagi, J., Yamada, M., Yasuda, M. \& Seki, M. Continuous particle separation in a microchannel having asymmetrically arranged multiple branches. Lab Chip 5, 778-784 (2005).

37. Yamada, M. \& Seki, M. Hydrodynamic filtration for on-chip particle concentration and classification utilizing microfluidics. Lab Chip 5, 1233-1239 (2005).

38. Groschl, M. Ultrasonic Separation of Suspended Particles - Part I : Fundamentals. Acustica 84, 432-447 (1998).

39. Lilliehorn, T. et al. Trapping of microparticles in the near field of an ultrasonic transducer. Ultrasonics 43, 293-303 (2005).

40. Bazou, D., Kuznetsova, L. A. \& Coakley, W. T. Physical enviroment of 2-D animal cell aggregates formed in a short pathlength ultrasound standing wave trap. Ultrasound Med. Biol. 31, 423-430 (2005).

41. Johnson, D. A. \& Feke, D. L. Methodology for fractionating suspended particles using ultrasonic standing wave and divided flow fields. Sep. Technol. 5, 251-258 (1995).

42. Yasuda, K., Umemura, S. \& Takeda, K. Concentration and Fractionation of Small Particles in Liquid by Ultrasound. Jpn. J. Appl. Phys. 34, 2715 (1995).

43. Petersson, F., Åberg, L., Swärd-Nilsson, A. M. \& Laurell, T. Free flow acoustophoresis: Microfluidic-based mode of particle and cell separation. Anal. Chem. 79, 5117-5123 (2007).

44. Bruus, H. Acoustofluidics 2: Perturbation theory and ultrasound resonance modes. Lab Chip 12, 20-28 (2012).

45. Doblhoff-Dier, O. et al. A Novel Ultrasonic Resonance Field Device for the Retention of Animal Cells. Biotechnol. Prog. 10, 428-432 (1994).

46. Hawkes, J. J. \& Coakley, W. T. Force field particle filter, combining ultrasound standing waves and laminar flow. Sensors Actuators B Chem. 75, 213-222 (2001).

47. Petersson, F., Nilsson, A., Jönsson, H. \& Laurell, T. Carrier Medium Exchange through Ultrasonic Particle Switching in Microfluidic Channels. Anal. Chem. 77, 1216-1221 (2005).

48. Kuznetsova, L. A. \& Coakley, W. T. Applications of ultrasound streaming and radiation force in biosensors. Biosens. Bioelectron. 22, 1567-1577 (2007).

49. Martin, S. P. et al. Spore and micro-particle capture on an immunosensor surface in an ultrasound standing wave system. Biosens. Bioelectron. 21, 758-767 (2005).

50. Laurell, T., Petersson, F. \& Nilsson, A. Chip integrated strategies for acoustic separation and manipulation of cells and particles. Chem. Soc. Rev. 36, 492-506 (2007).

51. Thévoz, P., Adams, J. D., Shea, H., Bruus, H. \& Soh, H. T. Acoustophoretic synchronization of mammalian cells in microchannels. Anal. Chem. 82, 3094-3098 (2010). 
52. Burguillos, M. a. et al. Microchannel Acoustophoresis does not Impact Survival or Function of Microglia, Leukocytes or Tumor Cells. PLoS One 8, 1-11 (2013).

53. Yang, A. H. J. \& Soh, H. T. Acoustophoretic Sorting of Viable Mammalian Cells in a Microfluidic Device. Anal. Chem. 84, 10756-10762 (2012).

54. Grenvall, C., Magnusson, C., Lilja, H. \& Laurell, T. Concurrent Isolation of Lymphocytes and Granulocytes Using Prefocused Free Flow Acoustophoresis. Anal. Chem. 5596-5604 (2015). doi:10.1021/acs.analchem.5b00370

55. Ward, M., Turner, P., DeJohn, M. \& Kaduchak, G. Fundamentals of acoustic cytometry. Curr. Protoc. Cytom. 1-12 (2009). doi:10.1002/0471142956.cy0122s49

56. Hawkes, J. J., Barber, R. W., Emerson, D. R. \& Coakley, W. T. Continuous cell washing and mixing driven by an ultrasound standing wave within a microfluidic channel. Lab Chip 4, 446-452 (2004).

57. Tenje, M. et al. Acoustophoretic removal of proteins from blood components. Biomed. Microdevices 17, 95 (2015).

58. Antfolk, M., Antfolk, C., Lilja, H., Laurell, T. \& Augustsson, P. A single inlet two-stage acoustophoresis chip enabling tumor cell enrichment from white blood cells. Lab Chip 15, 2102-2109 (2015).

59. Weinberg, R. A. The biology of Cancer. (Garland Science, Taylor \& Francis Group, LLC, 2014).

60. Li, P. et al. Acoustic separation of circulating tumor cells. Proc. Natl. Acad. Sci. U. S. A. 112, 4970-4975 (2015).

61. Ding, X. et al. Cell separation using tilted-angle standing surface acoustic waves. Proc. Natl. Acad. Sci. 111, 12992-12997 (2014).

62. Shields, C. W., Johnson, L. M., Gao, L. \& López, G. P. Elastomeric Negative Acoustic Contrast Particles for Capture, Acoustophoretic Transport, and Con fi nement of Cells in Micro fl uidic Systems. Langmuir 30, 3923-3927 (2014).

63. Phillips, R., Kondev, J., Theriot, J. \& Garcia, H. G. Physical Biology of the Cell. (Garland Science, Taylor \& Francis Group, LLC, 2013).

64. Alberts, B. et al. Molecular Biology of the Cell. (Garland Science, 2014).

65. Tien, H. T. \& Ottova-Leitmannova, A. Membrane Biophysics: As Viewed from Experimental Bilayer Lipid Membranes. (Elsevier Science B.V., 2000).

66. Liposomes, Lipid Bilayers and Model Membranes From Basic Research to Application. (CRC Press, Taylor \& Francis Group, LLC, 2014).

67. Bouvrais, H. Bending Rigidities of Lipid Bilayers: Their Determination and Main Inputs in Biophysical Studies. Advances in planar lipid bilayers and liposomes 15, (Elsevier Ltd, 2012).

68. Cell Lipids. (Academic Press, Inc., 1994).

69. Marsh, D. Handbook of Lipid Bilayers. (CRC Press, Taylor \& Francis Group, LLC, 2013). 
70. Lichtenberg, D. \& Barenholz, Y. in Methods of Biochemical Analysis, Volume 33 (ed. Glick, D.) 337-462 (John Wiley \& Sons, Inc., 1988).

71. M, E., M, D., Eeman, M. \& Deleu, M. From biological membranes to biomimetic model membranes. Focus (Madison). 14, 719-736 (2010).

72. Gorter, E. \& Grendel, F. On bimolecular layers of lipids on the chromocytes of the blood. J. Exp. Med. 41, 439-443 (1925).

73. Danielli, J. F. \& Davson, H. A contribution to the theory of permeability of thin films. $J$. Cell. Comp. Physiol. 5, 495-508 (1935).

74. Robertson, J. D. New Observations on the Ultrastructure of the Membranes of Frog Peripheral Nerve Fibers. J. Biophys. Biochem. Cytol. 3, 1043-1048 (1957).

75. Singer, S. J. \& Nicolson, G. L. The Fluid Mosaic Model of the Structure of Cell Membranes. Science (80-. ). 175, 720 LP-731 (1972).

76. Sezgin, E., Levental, I., Mayor, S. \& Eggeling, C. The mystery of membrane organization: composition, regulation and roles of lipid rafts. Nat Rev Mol Cell Biol advance on, (2017).

77. Tamm, L. K. \& McConnell, H. M. Supported phospholipid bilayers. Biophys. J. 47, 105113 (1985).

78. Sackmann, E. Supported Membranes: Scientific and Practical Applications. Science (80-. ). 271, 43 LP-48 (1996).

79. Menger, F. M. \& Keiper, J. S. Chemistry and physics of giant vesicles as biomembrane models. Curr. Opin. Chem. Biol. 2, 726-732 (1998).

80. Walde, P., Cosentino, K., Engel, H. \& Stano, P. Giant Vesicles: Preparations and Applications. ChemBioChem 11, 848-865 (2010).

81. Reeves, J. P. \& Dowben, R. M. Formation and properties of thin-walled phospholipid vesicles. J. Cell. Physiol. 73, 49-60 (1969).

82. Akashi, K., Miyata, H., Itoh, H. \& Kinosita, K. Preparation of giant liposomes in physiological conditions and their characterization under an optical microscope. Biophys. $J$. 71, 3242-3250 (1996).

83. Engelhardt, H., Duwe, H.P. \& Sackmann, E. Bilayer bending elasticity measured by Fourier analysis of thermally excited surface undulations of flaccid vesicles. J. Phys. Lett. 46, 395400 (1985).

84. Bivas, I., Hanusse, P., Bothorel, P., Lalanne, J. \& Aguerre-Chariol, O. An application of the optical microscopy to the determination of the curvature elastic modulus of biological and model membranes. J. Phys. Fr. 48, 855-867 (1987).

85. Evans, E. \& Rawicz, W. Entropy-driven tension and bending elasticity in condensed-fluid membranes. Phys. Rev. Lett. 64, 2094-2097 (1990).

86. Pan, J., Mills, T. T., Tristram-Nagle, S. \& Nagle, J. F. Cholesterol perturbs lipid bilayers nonuniversally. Phys. Rev. Lett. 100, 1-4 (2008).

87. Méléard, P. et al. Mechanical properties of model membranes studied from shape transformations of giant vesicles. Biochimie 80, 401-413 (1998). 
88. Rawicz, W., Olbrich, K. C., McIntosh, T., Needham, D. \& Evans, E. Effect of Chain Length and Unsaturation on Elasticity of Lipid Bilayers. Biophys. J. 79, 328-339 (2000).

89. Fernandez-Puente, L., Bivas, I., Mitov, M. D. \& Méléard, P. Temperature and Chain Length Effects on Bending Elasticity of Phosphatidylcholine Bilayers. Europhys. Lett. 28, 181-186 (2007).

90. Méléard, P. et al. Bending elasticities of model membranes: influences of temperature and sterol content. Biophys. J. 72, 2616-2629 (1997).

91. Dimova, R., Pouligny, B. \& Dietrich, C. Pretransitional Effects in Dimyristoylphosphatidylcholine Vesicle Membranes: Optical Dynamometry Study. Biophys. J. 79, 340-356 (2000).

92. Lenshof, A., Evander, M., Laurell, T. \& Nilsson, J. Acoustofluidics 5: Building microfluidic acoustic resonators. Lab Chip 12, 684-695 (2012).

93. Marai, L. \& Kuksis, A. Molecular species of lecithins from erythrocytes and plasma of man. J. Lipid Res. 10, 141-152 (1969).

94. Marcus, A. J., Ullman, H. L. \& Safier, L. B. Lipid composition of subcellular particles of human blood platelets. J. Lipid Res. 10, 108-114 (1969).

95. Pfleger, R. C., Anderson, N. G. \& Snyder, F. Lipid Class and Fatty Acid Composition of Rat Liver Plasma Membranes Isolated by Zonal Centrifugation. Biochemistry 7, 2826-2833 (1968).

96. Ejsing, C. S. et al. Global analysis of the yeast lipidome by quantitative shotgun mass spectrometry. Proc. Natl. Acad. Sci. \{U.S.A.\} 106, 2136-2141 (2009).

97. Sampaio, J. L. et al. Membrane lipidome of an epithelial cell line. Proc. Natl. Acad. Sci. U. S. A. 108, 1903-1907 (2011).

98. Greenspan, P., Mayer, E. P. \& Fowler, S. D. Nile red: a selective fluorescent stain for intracellular lipid droplets. J. Cell Biol. 100, 965 LP-973 (1985).

99. Yuan, T., Dong, J., Han, G. \& Wang, G. Polymer nanoparticles self-assembled from photo, $\mathrm{pH}$ - and thermo-responsive azobenzene-functionalized PDMAEMA. RSC Adv. 6, 1090410911 (2016).

100. Sanchez, S. A., Tricerri, M. A., Gunther, G. \& Gratton, E. Laurdan Generalized Polarization: from cuvette to microscope. Mod. Res. Educ. Top. Microsc. 2, 1007-1014 (2007).

101. Parasassi, T., De Stasio, G., d'Ubaldo, A. \& Gratton, E. Phase fluctuation in phospholipid membranes revealed by Laurdan fluorescence. Biophys. J. 57, 1179-1186 (1990).

102. Koning, A. J., Lum, P. Y., Williams, J. M. \& Wright, R. DiOC6 staining reveals organelle structure and dynamics in living yeast cells. Cell Motil. Cytoskeleton 25, 111-128 (1993).

103. Batzri, S. \& Korn, E. D. Single bilayer liposomes prepared without sonication. Biochim. Biophys. Acta 298, 1015-1019 (1973).

104. Pons, M., Foradada, M. \& Estelrich, J. Liposomes obtained by the ethanol injection method. Int. J. Pharm. 95, 51-56 (1993). 
105. Gao, K.-J. et al. Giant vesicle formation through self-assembly of chitooligosaccharidebased graft copolymers. Chem. Commun. 1449-1451 (2008). doi:10.1039/B718117E

106. Phillip, J. M., Aifuwa, I., Walston, J. \& Wirtz, D. The Mechanobiology of Aging. Annu. Rev. Biomed. Eng. 17, 113-41 (2015).

107. Lee, G. Y. H. \& Lim, C. T. Biomechanics approaches to studying human diseases. Trends Biotechnol. 25, 111-118 (2007).

108. Lam, W. a, Rosenbluth, M. J. \& Fletcher, D. A. Chemotherapy exposure increases leukemia cell stiffness Brief report Chemotherapy exposure increases leukemia cell stiffness. Blood 109, 3505-3508 (2007).

109. Di Carlo, D. A mechanical biomarker of cell state in medicine. J. Lab. Autom. 17, 32-42 (2012).

110. Suwanarusk, R. et al. The deformability of red blood cells parasitized by Plasmodium falciparum and P. vivax. J. Infect. Dis. 189, 190-194 (2004).

111. Barabino, G. a, Platt, M. O. \& Kaul, D. K. Sickle cell biomechanics. Annu. Rev. Biomed. Eng. 12, 345-367 (2010).

112. Wood, C. D. et al. Alignment of particles in microfluidic systems using standing surface acoustic waves. Appl. Phys. Lett. 92, 10-13 (2008).

113. Fernandez Rivas, D., Cintas, P. \& Gardeniers, H. J. G. E. Merging microfluidics and sonochemistry: towards greener and more efficient micro-sono-reactors. Chem. Commun. 48, 10935 (2012).

114. Franke, T., Braunmüller, S., Schmid, L., Wixforth, a \& Weitz, D. a. Surface acoustic wave actuated cell sorting (SAWACS). Lab Chip 10, 789-794 (2010).

115. Nam, J., Lim, H., Kim, D. \& Shin, S. Separation of platelets from whole blood using standing surface acoustic waves in a microchannel. Lab Chip 11, 3361-4 (2011).

116. Ding, X. et al. Standing surface acoustic wave (SSAW) based multichannel cell sorting. Lab Chip 12, 4228 (2012).

117. Faria, E. C. et al. Measurement of elastic properties of prostate cancer cells using AFM. Analyst 133, 1498-1500 (2008).

118. Tan, S. C. W. et al. Viscoelastic behaviour of human mesenchymal stem cells. BMC Cell Biol. 9, 40 (2008).

119. Williamson, J. R., Shanahan, M. O. \& Hochmuth, R. M. The Influence of Temperature on Red Blood Cell Deformability. Blood 46, 611-625 (1975).

120. Yang, T. et al. Investigation of temperature effect on cell mechanics by optofluidic microchips. Biomed. Opt. Express 6, 2991-2996 (2015).

121. Chapman, D., Williams, R. M. \& Ladbrooke, B. D. Physical studies of phospholipids. VI. Thermotropic and lyotropic mesomorphism of some 1,2-diacyl-phosphatidylcholines (lecithins). Chem. Phys. Lipids 1, 445-475 (1967).

122. Tarini, M., Cignoni, P. \& Montani, C. Ambient occlusion and edge cueing for enhancing real time molecular visualization. IEEE Trans. Vis. Comput. Graph. 12, 1237-1244 (2006). 
123. Dolatmoradi, A. \& El-Zahab, B. Thermally-assisted ultrasonic separation of giant vesicles. Lab Chip 16, 3449-3453 (2016).

124. Seto, H., Yamada, N. L., Nagao, M., Hishida, M. \& Takeda, T. Bending modulus of lipid bilayers in a liquid-crystalline phase including an anomalous swelling regime estimated by neutron spin echo experiments. Eur. Phys. J. E. Soft Matter 26, 217-23 (2008).

125. Yi, Z., Nagao, M. \& Bossev, D. P. Bending elasticity of saturated and monounsaturated phospholipid membranes studied by the neutron spin echo technique. J. Phys. Condens. Matter 21, 155104 (2009).

126. Woodka, A. C., Butler, P. D., Porcar, L., Farago, B. \& Nagao, M. Lipid Bilayers and Membrane Dynamics: Insight into Thickness Fluctuations. Phys. Rev. Lett. 109, 58102 (2012).

127. Needham, D. \& Evans, E. Structure and mechanical properties of giant lipid (DMPC) vesicle bilayers from 20 degrees $\mathrm{C}$ below to 10 degrees $\mathrm{C}$ above the liquid crystal-crystalline phase transition at 24 degrees C. Biochemistry 27, 8261-8269 (1988).

128. Lewis, R. N., Mak, N. \& McElhaney, R. N. A differential scanning calorimetric study of the thermotropic phase behavior of model membranes composed of phosphatidylcholines containing linear saturated fatty acyl chains. Biochemistry 26, 6118-6126 (1987).

129. Lee, C. H., Lin, W. C. \& Wang, J. All-optical measurements of the bending rigidity of lipidvesicle membranes across structural phase transitions. Phys. Rev. E. Stat. Nonlin. Soft Matter Phys. 64, 20901 (2001).

130. Heimburg, T. Mechanical aspects of membrane thermodynamics. Estimation of the mechanical properties of lipid membranes close to the chain melting transition from calorimetry. Biochim. Biophys. Acta-Biomembr. 1415, 147-162 (1998).

131. Krivanek, R., Rybar, P., Prenner, E. J., McElhaney, R. N. \& Hianik, T. Interaction of the antimicrobial peptide gramicidin $\mathrm{S}$ with dimyristoyl-phosphatidylcholine bilayer membranes: A densitometry and sound velocimetry study. Biochim. Biophys. Acta Biomembr. 1510, 452-463 (2001).

132. Heller, H. Simulation einer lipidmembran auf einem parallelrechner. (Technical University of Munich, 1993).

133. Heller, H., Schaefer, M. \& Schulten, K. Molecular dynamics simulation of a bilayer of 200 lipids in the gel and in the liquid crystal phase. J. Phys. Chem. 97, 8343-8360 (1993).

134. Heimburg, T. \& Jackson, A. D. On soliton propagation in biomembranes and nerves. Proc. Natl. Acad. Sci. U. S. A. 102, 9790-9795 (2005).

135. Mabrey, S. \& Sturtevant, J. M. Investigation of phase transitions of lipids and lipid mixtures by sensitivity differential scanning calorimetry. Proc. Natl. Acad. Sci. U. S. A. 73, 38623866 (1976).

136. Cheng, W. H. A theoretical description of phase diagrams for nonideal lipid mixtures. Biochim. Biophys. Acta 600, 358-366 (1980).

137. Dolatmoradi, A. \& El-Zahab, B. Thermally-assisted acoustophoresis as a new stiffnessbased separation method. in 10061, (2017). 
138. Wiklund, M. Acoustofluidics 12: Biocompatibility and cell viability in microfluidic acoustic resonators. Lab Chip 12, 2018-28 (2012).

139. Robert B. Gennis. Biomembranes: Molecular Structure and Function. (Springer-Verlag New York, 1989). doi:10.1007/978-1-4757-2065-5

140. Shu Chien. Red cell deformability and its relevance to blood flow. Annu. Rev. Physiol. 49, 177-192 (1987).

141. Schaefer, A. \& Hordijk, P. L. Cell stiffness-induced mechanosignaling - a key driver of leukocyte transendothelial migration. J. Cell Sci. 128, 2221-30 (2015).

142. Nakazawa, I. \& Iwaizumi, M. A role of the cancer cell membrane fluidity in the cancer metastases: an ESR study. Tohoku J. Exp. Med. 157, 193-198 (1989).

143. Swaminathan, V. et al. Mechanical Stiffness grades metastatic potential in patient tumor cells and in cancer cell lines. Cancer Res. 71, 5075-5080 (2011).

144. Xu, W. et al. Cell Stiffness Is a Biomarker of the Metastatic Potential of Ovarian Cancer Cells. PLoS One 7, e46609 (2012).

145. Fenner, J. et al. Macroscopic stiffness of breast tumors predicts metastasis. Sci. Rep. 4, 5512 (2014).

146. Liga, A., Vliegenthart, A. D. B., Oosthuyzen, W., Dear, J. W. \& Kersaudy-Kerhoas, M. Exosome isolation: a microfluidic road-map. Lab Chip 15, 2388-2394 (2015).

147. He, M., Crow, J., Roth, M., Zeng, Y. \& Godwin, A. K. Integrated immunoisolation and protein analysis of circulating exosomes using microfluidic technology. Lab Chip 14, 37733780 (2014).

148. Thery, C. Cancer: Diagnosis by extracellular vesicles. Nature 523, 161-162 (2015).

149. EL Andaloussi, S., Mager, I., Breakefield, X. O. \& Wood, M. J. A. Extracellular vesicles: biology and emerging therapeutic opportunities. Nat Rev Drug Discov 12, 347-357 (2013).

150. De Toro, J., Herschlik, L., Waldner, C. \& Mongini, C. Emerging Roles of Exosomes in Normal and Pathological Conditions: New Insights for Diagnosis and Therapeutic Applications . Frontiers in Immunology 6, 203 (2015).

151. Dolatmoradi, A., Mirtaheri, E. \& El-Zahab, B. Thermo-acoustofluidic separation of vesicles based on cholesterol content. Lab Chip 17, 1332-1339 (2017).

152. Heimburg, T. Thermal Biophysics of Membranes. (WILEY-VCH Verlag GmbH \& Co. $\mathrm{KGaA}, 2007)$.

153. Tziakas, D. N. et al. Total Cholesterol Content of Erythrocyte Membranes Is Increased in Patients With Acute Coronary Syndrome. A New Marker of Clinical Instability? J. Am. Coll. Cardiol. 49, 2081-2089 (2007).

154. Lipids and Cellular Membranes in Amyloid Diseases. (WILEY-VCH Verlag \& Co. KGaA, 2011).

155. Van Blitterswijk, W. I. M. J., Hilkmann, H. \& Hengeveld, T. Differences in membrane lipid composition and fluidity of transplanted GRSL lymphoma cells, depending on their site of growth in the mouse. Biochim. Biophys. Acta 778, 521-529 (1984). 
156. Kojima, K. Molecular aspects of the plasma membrane in tumor cells. Nagoya J Med Sci $1-18$ (1993).

157. Li, Y. C., Park, M. J., Ye, S.-K., Kim, C.-W. \& Kim, Y.-N. Elevated levels of cholesterolrich lipid rafts in cancer cells are correlated with apoptosis sensitivity induced by cholesterol-depleting agents. Am. J. Pathol. 168, 1107-1118-1405 (2006).

158. Hager, M. H., Solomon, K. R. \& Freeman, M. R. The role of cholesterol in prostate cancer. Curr. Opin. Clin. Nutr. Metab. Care 9, 379-385 (2006).

159. Cortes, V. a. et al. Advances in the physiological and pathological implications of cholesterol. Biol. Rev. 88, 825-843 (2013).

160. Nicolau CT, Teitel, P., Fotino, M., Butoianu, E. \& Taigar, S. Alterations of erythrocyte plasticity in blood diseases. Sangre (Barc) 10, 282-288 (1964).

161. Allard, C., Mohandas, N. \& Bessis, M. in Red Cell Rheology (eds. Bessis, M., Shohet, S. B. \& Mohandas, N.) 209-221 (Springer Berlin Heidelberg, 1978). doi:10.1007/978-3-64267059-6_16

162. Ballas, S. K. et al. Rheologic predictors of the severity of the painful sickle cell crisis. Blood 72, 1216 LP-1223 (1988).

163. Bessis, M. \& Mohandas, N. Laser diffraction patterns of sickle cells in fluid shear fields. Blood Cells 3, 229-239 (1977).

164. Lande, W. M. et al. The incidence of painful crisis in homozygous sickle cell disease: correlation with red cell deformability. Blood 72, 2056 LP-2059 (1988).

165. Dodds, A. J. et al. Changes in red cell deformability and other haemorrheological variables after myocardial infarction. Br. Heart J. 44, 508-511 (1980).

166. Dormandy, J., Boyd, M. \& Ernst, E. Red cell filterability after myocardial infarction. Scand. J. Clin. Lab. Invest. 41, 195-198 (1981).

167. Brown, P. \& Harrison, M. J. G. Changes in blood filtrability and platelet aggregability in patients with aortic valve replacements. Clin. Hemorheol. 9, 139-147 (1989).

168. Ekeström, S., Koul, B. L. \& Sonnenfeld, T. Decreased Red Cell Deformability Following Open-Heart Surgery. Scand. J. Thorac. Cardiovasc. Surg. 17, 41-44 (1983).

169. Hirayama, T., Yamaguchi, H., Allers, M. \& Roberts, D. Evaluation of Red Cell Damage During Cardiopulmonary Bypass. Scand. J. Thorac. Cardiovasc. Surg. 19, 263-265 (1985).

170. Hirayama, T., Yamaguchi, H., Allers, M., Roberts, D. \& William-Olsson, G. Changes in Red Cell Deformability Associated with Anaesthesia and Cardiopulmonary Bypass in Open-Heart Surgery. Scand. J. Thorac. Cardiovasc. Surg. 19, 257-262 (1985).

171. Bareford, D., Stone, P. C. W., Caldwell, N. M. \& Stuart, J. Erythrocyte morphology as a determinant of abnormal erythrocyte deformability in liver disease. Clin. Hemorheol. 5, 473-481 (1985).

172. Bareford, D. et al. Erythrocyte deformability in chronic renal failure. Clin. Hemorheol. 6, 501-510 (1986). 
173. Decamps, A. et al. Red cell filterability and chronic renal failure. Scand. J. Clin. Lab. Invest. 41, 177-179 (1981).

174. Kikuchi, Y. et al. Red Blood Cell Deformability in Renal Failure. Nephron 30, 8-14 (1982).

175. Inauen, W., Stäubli, M., Descoeudres, C., Galeazzi, R. L. \& Straub, P. W. Erythrocyte deformability in dialysed and non-dialysed uraemic patients. Eur. J. Clin. Invest. 12, 173176 (1982).

176. Cecchin, E., De Marchi, S., Panarello, G. \& De Angelis, V. Rheological Abnormalities of Erythrocyte Deformability and Increased Glycosylation of Hemoglobin in the Nephrotic Syndrome. Am. J. Nephrol. 7, 18-21 (1987).

177. McMillan, D. E., Utterback, N. G. \& Puma, J. La. Reduced erythrocyte deformability in diabetes. Diabetes 27, 895-901 (1978).

178. Schmid-Schönbein, H. \& Volger, E. Red-cell aggregation and red-cell deformability in diabetes. Diabetes 25, 897-902 (1976).

179. Ernst, E. \& Matrai, A. Altered Red and White Blood Cell Rheology in Type II Diabetes. Diabetes 35, 1412 LP-1415 (1986).

180. Juhan, I. et al. Effects of insulin on erythrocyte deformability in diabetics - relationship between erythrocyte deformability and platelet aggregation. Scand. J. Clin. Lab. Invest. 41, 159-164 (1981).

181. Juhan, I. et al. Abnormalities of erythrocyte deformability and platelet aggregation in insulin-dependent diabetics corrected by insulin in vivo and in vitro. Lancet 319, 535-537 (1982).

182. Ozanne, P. et al. Whole blood filterability in diabetics. Influence of age, complications and duration of diabetes. Scand. J. Clin. Lab. Invest. 41, 259-260 (1981).

183. Lowe, G. D. O., Drummond, M. M., Belch, J. J. F., Lowe, J. M. \& MacCuish, A. C., \& Manderson, W. G. in Microvascular Research 17, S58 (Academic Press Inc JNL-COMP Subscriptions, 1979).

184. Cranston, H. A. et al. Plasmodium falciparum maturation abolishes physiologic red cell deformability. Science (80-. ). 223, 400 LP-403 (1984).

185. Nash, G., O’Brien, E., Gordon-Smith, E. \& Dormandy, J. Abnormalities in the mechanical properties of red blood cells caused by Plasmodium falciparum. Blood 74, 855 LP-861 (1989).

186. Watała, C., Kordacka, M., Loba, A., Jóźwiak, Z. \& Nowak, S. Analysis of membrane fluidity alterations and lipid disorders in type I diabetic children and adolescents. Acta Diabetol. Lat. 24, 141-8 (1987).

187. Tetsuro Kamada \& Otsuji, S. Lower Levels of Erythrocyte Membrane Fluidity in Diabetic Patients: A Spin Label Study. Diabetes 32, 585-591 (1983).

188. Koichi Shiraishi, Matsuzaki, S., Ishida, H. \& Nakazawa, H. Impaired Erythrocyte Deformability and Membrane Fluidity in Alcoholic Liver Disease: Participation in Disturbed Hepatic Microcirculation. Alcohol Alcohol. 28, 59-64 (1993). 
189. Kolanjiappan, K., Manoharan, S. \& Kayalvizhi, M. Measurement of erythrocyte lipids, lipid peroxidation, antioxidants and osmotic fragility in cervical cancer patients. Clin. Chim. Acta 326, 143-149 (2002).

190. Roses, A. D. \& Appel, S. H. Phosphorylation of component 'a' of the human erythrocyte membrane in myotonic muscular dystrophy. J. Membr. Biol. 20, 51-58 (1975).

191. Górnicki, A. \& Gutsze, A. Erythrocyte membrane fluidity changes in psoriasis: an EPR study. J. Dermatol. Sci. 27, 27-30 (2001).

192. Yao, J. K., van Kammen, D. P. \& Welker, J. A. Red blood cell membrane dynamics in schizophrenia. II. Fatty acid composition. Schizophr. Res. 13, 217-226 (1994).

193. Tongyai, S. et al. Mechanism of increased erythrocyte membrane fluidity during magnesium deficiency in weanling rats. Am. J. Physiol. - Cell Physiol. 257, C270-C276 (1989).

194. Almeida, P. F., Vaz, W. L. \& Thompson, T. E. Lateral diffusion in the liquid phases of dimyristoylphosphatidylcholine/cholesterol lipid bilayers: a free volume analysis. Biochemistry 31, 6739-6747 (1992).

195. Sankaram, M. B. \& Thompson, T. E. Cholesterol-induced fluid-phase immiscibility in membranes. Proc. Natl. Acad. Sci. U. S. A. 88, 8686-8690 (1991).

196. Reyes Mateo, C., Ulises Acuña, a \& Brochon, J. C. Liquid-crystalline phases of cholesterol/lipid bilayers as revealed by the fluorescence of trans-parinaric acid. Biophys. $J$. 68, 978-987 (1995).

197. Hofsäß, C., Lindahl, E. \& Edholm, O. Molecular Dynamics Simulations of Phospholipid Bilayers with Cholesterol. Biophys. J. 84, 2192-2206 (2003).

198. Henriksen, J., Rowat, A. C. \& Ipsen, J. H. Vesicle fluctuation analysis of the effects of sterols on membrane bending rigidity. Eur. Biophys. J. 33, 732-741 (2004).

199. Gracià, R. S., Bezlyepkina, N., Knorr, R. L., Lipowsky, R. \& Dimova, R. Effect of cholesterol on the rigidity of saturated and unsaturated membranes: fluctuation and electrodeformation analysis of giant vesicles. Soft Matter 6, 1472 (2010).

200. Tierney, K. J., Block, D. E. \& Longo, M. L. Elasticity and phase behavior of DPPC membrane modulated by cholesterol, ergosterol, and ethanol. Biophys. J. 89, 2481-2493 (2005).

201. Halstenberg, S., Heimburg, T., Hianik, T., Kaatze, U. \& Krivanek, R. Cholesterol-induced variations in the volume and enthalpy fluctuations of lipid bilayers. Biophys $J$ 75, 264-271 (1998).

202. Krivanek, R., Okoro, L. \& Winter, R. Effect of cholesterol and ergosterol on the compressibility and volume fluctuations of phospholipid-sterol bilayers in the critical point region: a molecular acoustic and calorimetric study. Biophys. J. 94, 3538-48 (2008).

203. Gor'kov, L. P. On the forces acting on a small particle in an acoustical field in an ideal fluid. Sov. Phys. Dokl. 6, 773-775 (1962).

204. Yosioka, K. \& Kawasima, Y. Acoustic radiation pressure on a compressible sphere. Acta Acust. united with Acust. 5, 167-173 (1955). 


\section{Appendix I}

Table AI1 - Lipids used in the preparation of the vesicles.

\begin{tabular}{|c|c|c|c|}
\hline Lipid & $\begin{array}{l}\text { Molecular } \\
\text { Formula }\end{array}$ & Synonyms & $\begin{array}{l}\text { Molecular } \\
\text { Weight }\end{array}$ \\
\hline Lecithin & $\mathrm{N} / \mathrm{A}^{*}$ & $\begin{array}{l}\text { 1,2-Diacyl-sn-glycero-3-phosphocholine, L- } \alpha \text { - } \\
\text { phosphatidylcholine, Egg PC, EPC, }\end{array}$ & $\sim 768 \mathrm{~g} / \mathrm{mol}$ \\
\hline DLPC & $\mathrm{C}_{32} \mathrm{H}_{64} \mathrm{NO}_{8} \mathrm{P}$ & $\begin{array}{l}\text { 1,2-Didodecanoyl-sn-glycero-3-phosphocholine, 12:0 PC, } \\
\text { PC(12:0/12:0) }\end{array}$ & $621.83 \mathrm{~g} / \mathrm{mol}$ \\
\hline DMPC & $\mathrm{C}_{36} \mathrm{H}_{72} \mathrm{NO}_{8} \mathrm{P}$ & $\begin{array}{l}\text { 1,2-Ditetradecanoyl-sn-glycero-3-phosphocholine, 14:0 } \\
\text { PC, PC(14:0/14:0) }\end{array}$ & $677.93 \mathrm{~g} / \mathrm{mol}$ \\
\hline DPPC & $\mathrm{C}_{40} \mathrm{H}_{80} \mathrm{NO}_{8} \mathrm{P}$ & $\begin{array}{l}\text { 1,2-Dihexadecanoyl-sn-glycero-3-phosphocholine, 16:0 } \\
\text { PC, PC(16:0/16:0) }\end{array}$ & $734.04 \mathrm{~g} / \mathrm{mol}$ \\
\hline POPC & $\mathrm{C}_{42} \mathrm{H}_{82} \mathrm{NO}_{8} \mathrm{P}$ & $\begin{array}{l}\text { 1-Hexadecanoyl-2-(9Z-octadecenoyl)-sn-glycero-3- } \\
\text { phosphocholine, 16:0-18:1 PC, PC(16:0/18:1(9Z)) }\end{array}$ & $760.08 \mathrm{~g} / \mathrm{mol}$ \\
\hline DOPC & $\mathrm{C}_{44} \mathrm{H}_{84} \mathrm{NO}_{8} \mathrm{P}$ & $\begin{array}{l}\text { 1,2-di-(9Z-octadecenoyl)-sn-glycero-3-phosphocholine, } \\
\text { 18:1 ( } \Delta 9 \text {-Cis) PC, PC(18:1(9Z)/18:1(9Z)) }\end{array}$ & $786.11 \mathrm{~g} / \mathrm{mol}$ \\
\hline Cholesterol & $\mathrm{C}_{27} \mathrm{H}_{46} \mathrm{O}$ & $3 \beta$-Hydroxy-5-cholestene, 5-Cholesten-3 $\beta$-ol & $386.65 \mathrm{~g} / \mathrm{mol}$ \\
\hline
\end{tabular}

*The chemical formula of lecithin is determined based on the fatty acid distribution in the product. 


\section{Appendix II}

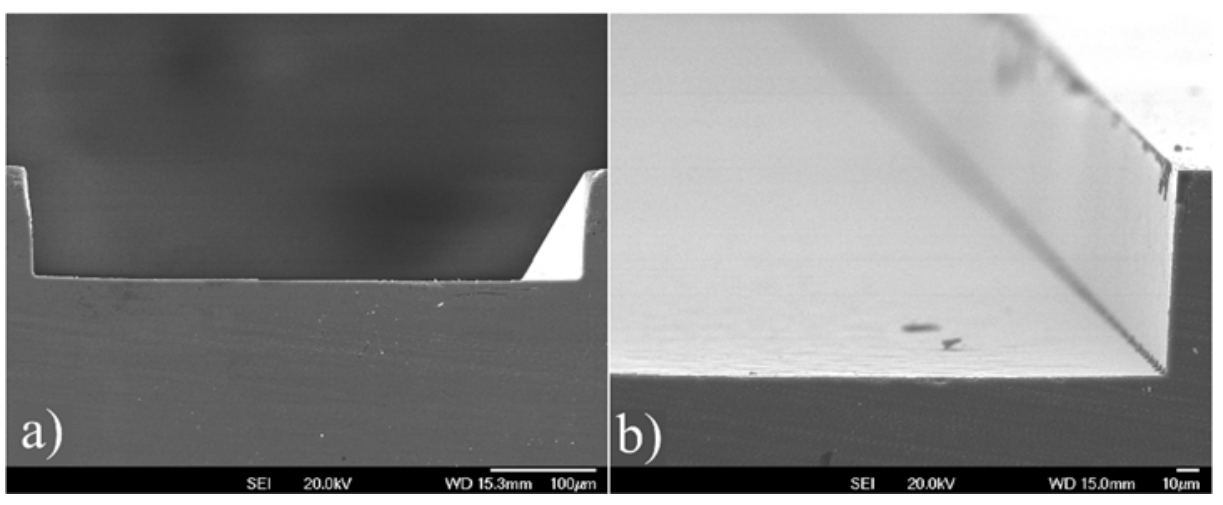

Figure AII1 - Scanning electron micrographs of the microfluidic channel's cross section. 


\section{Appendix III}

The size distribution profiles estimated using the ImageJ software.

\begin{tabular}{|c|c|c|}
\hline 9 & 13 & 15 \\
\hline 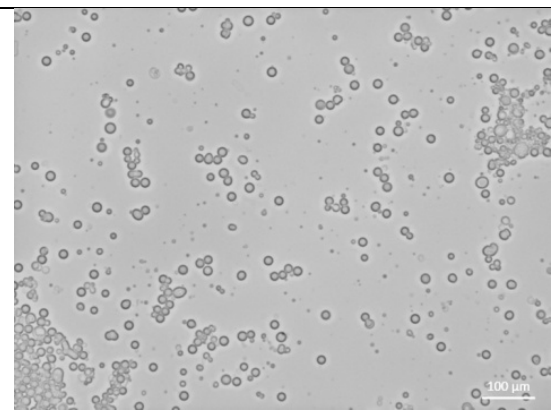 & 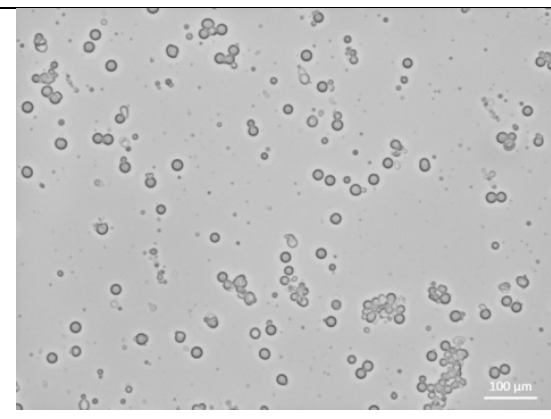 & 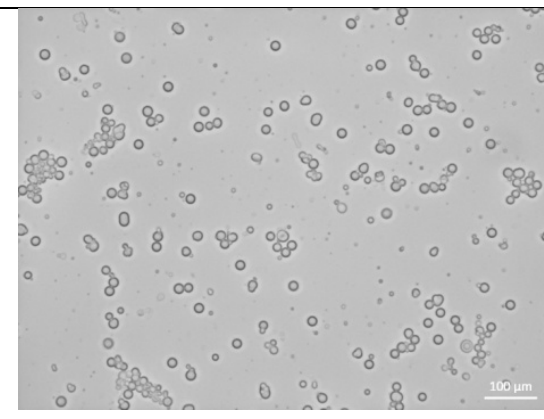 \\
\hline Ave $=11.57$ & Ave $=11.50$ & Ave $=12.05$ \\
\hline 17 & 21 & 23 \\
\hline 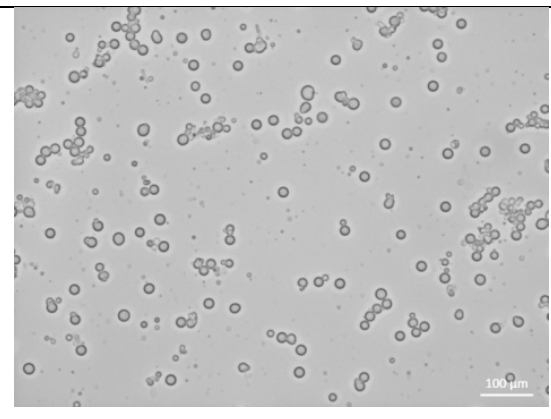 & 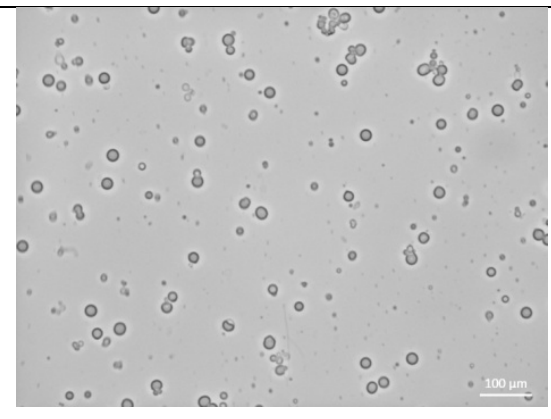 & $\begin{array}{ccccc}0 & 0 & 0 & 0 \\
0 & 0 & 0 & 0 \\
0 & 0 & 0 & 0 \\
0 & 0 & 0 & 0 & 0 \\
0 & 0 & 0 & 0 & 0 \\
0 & 0 & 0 & 0 \\
0 & 0 & 0 & 0\end{array}$ \\
\hline Ave $=11.58$ & Ave $=10.42$ & Ave $=10.28$ \\
\hline
\end{tabular}




\begin{tabular}{|c|c|c|}
\hline 9 & 13 & 15 \\
\hline 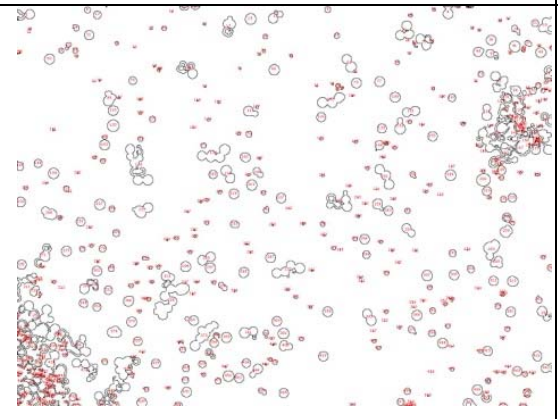 & 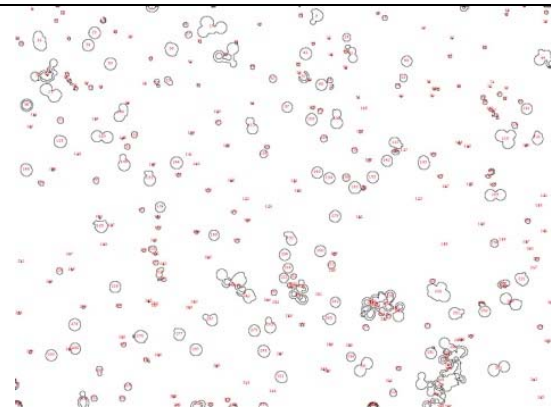 & 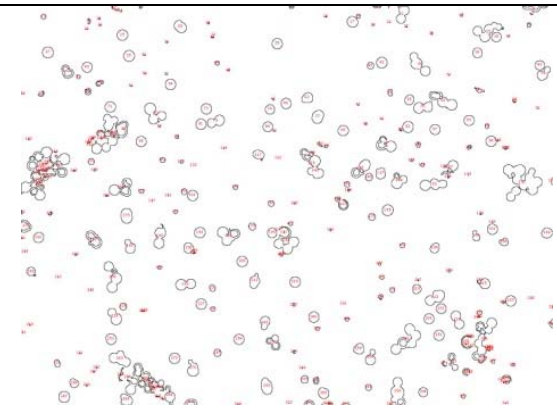 \\
\hline $\mathrm{Ave}=11.57$ & Ave $=11.50$ & $\mathrm{Ave}=12.05$ \\
\hline 17 & 21 & 23 \\
\hline 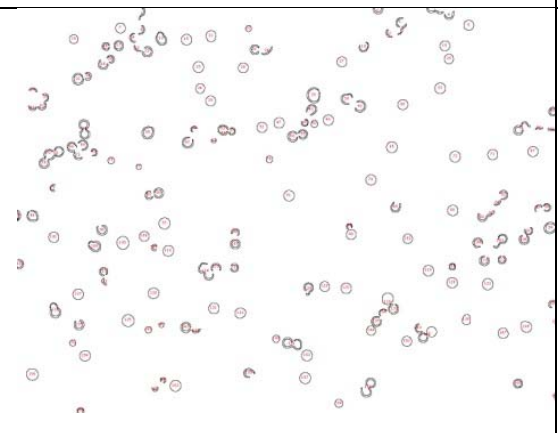 & 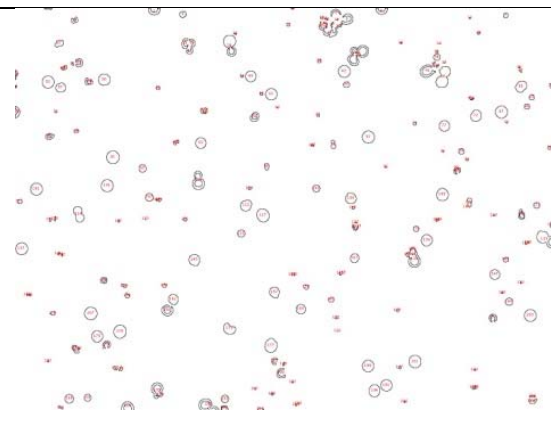 & 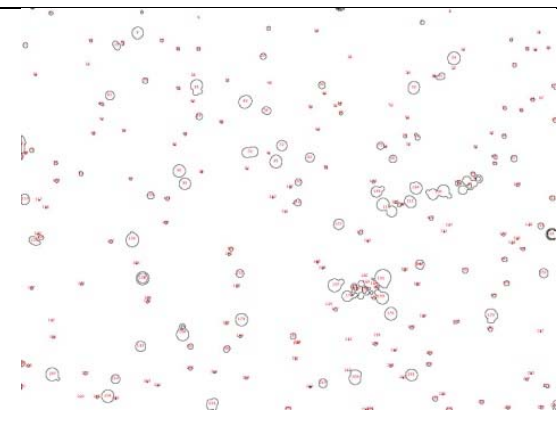 \\
\hline $\mathrm{Ave}=11.58$ & Ave $=10.42$ & $A v e=10.28$ \\
\hline
\end{tabular}




\section{Appendix IV}

\section{Calculation of Acoustic Contrast Factor}

In a standing acoustic field, the direction towards which a compressible spherical particle migrates is determined by the acoustic radiation force, described as: ${ }^{203}$

$\boldsymbol{F}^{\mathrm{rad}}=-\boldsymbol{\nabla} U^{\mathrm{rad}}$

where $U^{r a d}$ is the acoustic potential and the diameter of the particle is assumed to be much smaller than the wavelength of the applied acoustic wave. For a one-dimensional planar standing wave, if the induced acoustic pressure amplitude is assumed to be of the form:

$p(x, y, z)=p_{\mathrm{a}} \cos \left(k_{x} x\right)$

in which $k_{x}=2 \pi / \lambda$ and $\lambda=2 w, w$ being the channel width, the acoustic radiation force can be then reduced to the one-dimensional form: ${ }^{204}$

$F_{x}^{\mathrm{rad}}=4 \pi \phi(\rho, \beta) k_{x} r^{3} E_{\mathrm{ac}} \sin \left(2 k_{x} x\right)$

in which the acoustic radiation force along the direction $x$ (the direction perpendicular to the flow) is directly related to the particle radius, $r$, acoustic energy density, $E_{\mathrm{ac}}$, and the acoustic contrast factor, $\phi(\rho, \beta)$, which is a function of the compressibility and density of the particles/vesicles and medium: ${ }^{11}$

$\phi(\rho, \beta)=\frac{1}{3}\left[\frac{5 \rho_{\mathrm{v}}-2 \rho_{0}}{2 \rho_{\mathrm{v}}+\rho_{0}}-\frac{\beta_{\mathrm{v}}}{\beta_{0}}\right]$

where $\rho_{\mathrm{v}}$ is the density of vesicles, $\rho_{0}$ the density of the medium, and $\beta_{\mathrm{v}}$ and $\beta_{0}$ are the compressibility values for the vesicles and medium, respectively.

Since the internal volume of giant vesicles is extremely large compared to its enclosing membrane, it is safe to assume that the density ratio is very close to unity. Therefore, $\phi$ is here is mainly dependent on the compressibility ratio of the vesicles and medium. 
The elastic properties of aqueous media and vesicle suspensions can be evaluated by using the relationship: ${ }^{130}$

$\beta_{\mathrm{S}}=\frac{1}{\rho u^{2}}$

where $\beta_{\mathrm{S}}, u$, and $\rho$ are the adiabatic compressibility, the speed of sound, and the density of the vesicles suspension, respectively. The parameter usually determined in ultrasonic velocimetry experiments is sound velocity number $([u])$, which is defined by the equation: ${ }^{130}$

$[u]=\frac{u-u_{0}}{u_{0}[L]}$

where, $u$ and $u_{0}$ denote the speed of sound in the vesicle suspension and in the solvent, respectively, and $[L]$ is the lipid concentration, usually given in $\mathrm{mg} \cdot \mathrm{cm}^{-3}$.

The basic value of $[u]$ as a function of temperature is determined by sound velocity experiments. The density of the vesicles in the solution $(\rho)$, determined by densitometry experiments, is also used to determine the apparent specific partial volume of the solution $\left(\varphi_{\mathrm{V}}\right)$, which is defined by: ${ }^{131}$

$\varphi_{\mathrm{V}}=\frac{\left[1-\left(\rho-\rho_{0}\right) /[L]\right]}{\rho_{0}}=\frac{1}{\rho_{0}}-[\rho]$

whereby $\rho_{0}$ denotes the density of the solvent and $[\rho]=\left(\rho-\rho_{0}\right) /\left(\rho_{0}[L]\right)$ denotes the concentration increment of density. Based on the values of the specific volume and the sound velocity concentration increment, one could estimate the changes of the specific adiabatic compressibility $\left(\varphi_{\mathrm{K}} / \beta_{0}\right)$ of the vesicles during the phase transition by the following equation: ${ }^{131}$

$\frac{\varphi_{\mathrm{K}}}{\beta_{0}}=-2[u]-\frac{1}{\rho_{0}}+2 \varphi_{\mathrm{V}}$

whereby $\beta_{0}$ is the coefficient of adiabatic compressibility and $\varphi_{\mathrm{K}} / \beta_{0}$ is the changes of the volume compressibility of the vesicles suspension relative to the solvent. The apparent specific compressibility can be also expressed as: ${ }^{131}$

$\frac{\varphi_{\mathrm{K}}}{\beta_{0}}=\varphi_{\mathrm{V}}+\left[\beta_{\mathrm{S}}\right]$ 
where $\left[\beta_{\mathrm{S}}\right]=\left(\beta_{\mathrm{S}}-\beta_{\mathrm{S} 0}\right) /\left(\beta_{\mathrm{S} 0}[L]\right)$ is the concentration increment of the adiabatic compressibility, in which $\beta_{\mathrm{S}}$ and $\beta_{\mathrm{S} 0}$ denote the adiabatic compressibility of the vesicles suspension and solvent, respectively. The calculated value of $\beta_{\mathrm{S}}$ would represent the compressibility of the suspension, which can be then used to find the value of the compressibility of the vesicles by:

$\beta_{\mathrm{S}, \text { suspension }}=f_{0} \cdot \beta_{\mathrm{S} 0}+f_{\mathrm{v}} \cdot \beta_{\mathrm{S}, \mathrm{v}}$

in which $f_{0}$ and $f_{\mathrm{v}}$ are the volume fractions of the water and vesicles, respectively, and $\beta_{\mathrm{S} 0}$ and $\beta_{\mathrm{S}, \mathrm{v}}$ denote the compressibilities of water and vesicles, respectively. 
VITA

\section{ATA DOLATMORADI}

1986

2008

2011

2013-2016

2015

2015-2017

2016-2017

2017

2017
Born, Mashhad, Iran

B.S., Materials Engineering

Ferdowsi University of Mashhad

Mashhad, Iran

M.S., Materials Engineering

University of Tehran

Tehran, Iran

Graduate Teaching Assistant

Florida International University, Miami, Florida

NIH RISE Biomedical Research Initiative Award Florida International University, Miami, Florida

Doctoral Candidate in Materials Science and Engineering Florida International University, Miami, Florida

Doctoral Candidate and Dissertation Year Fellow

Florida International University, Miami, Florida

SPIE Best Paper Award and Travel Grant

Photonics West

San Francisco, California

Finalist (Ongoing competition)

Florida Blue Healthcare Innovation Pitch Competition

Tampa, Florida

\section{PUBLICATIONS AND PRESENTATIONS}

El-Zahab, B., and Dolatmoradi, A. (2017). Thermally-Assisted Acoustic Separation of Cells Based on Their Stiffness, Patent Application (US 15/463,164).

Dolatmoradi, A., Mirtaheri, E., and El-Zahab, B. (2017). Thermo-Acoustofluidic Separation of Vesicles Based on Cholesterol Content, Lab on a Chip, 17: 1332-1339. 
Dolatmoradi, A., and El-Zahab, B. (2017). Thermally-Assisted Acoustophoresis as a New Stiffness-Based Separation Method, SPIE BiOS, 10061: 1121-1124.

Dolatmoradi, A., and El-Zahab, B. (2016). Thermally-Assisted Ultrasonic Separation of Giant Vesicles, Lab on a Chip, 16: 3449-3453.

Dolatmoradi, A., and El-Zahab, B. (November, 2016). Stiffness-Based Separation of Giant Liposomes via Acoustophoresis. Paper presented at the MRS Fall Meeting \& Exhibit, Boston, Massachusetts.

Dolatmoradi, A., and El-Zahab, B. (March, 2016). Investigating Acoustophoresis for the Separation of Circulating Tumor Cell Analogs. Paper presented at the $18^{\text {th }}$ Annual Biomedical and Comparative Immunology (BCI) Symposium, Miami, Florida.

Dolatmoradi, A., and El-Zahab, B. (March, 2015). Behavior of Giant Vesicles in Acoustically-Excited Microchannels. Paper presented at the TMS Annual Meeting \& Exhibition, Orlando, Florida. 